Building Blocks: Conceptualizing the True Socio-Political Potential in Blockchain's Facilitation of Self-Sovereign Digital Identity and Decentralized Organization

\title{
by
}

\section{Jack Smye}

A thesis submitted to the Faculty of Graduate and Postdoctoral Affairs in partial fulfillment of the requirements for the degree of

\author{
Master of Arts \\ in \\ Political Economy \\ Carleton University \\ Ottawa, Ontario
}

(C) 2019

Jack Smye 


\begin{abstract}
Blockchain has been revered as a revolutionary technology capable of facilitating the transition to new economic and political systems premised on decentralization. In order to do this, however, it must transcend the contemporary digital landscape in its facilitation of digital identity - which is perhaps its most promising potential in that it can enable what is referred to as self-sovereign identity. The dominant line of thinking regarding this potential, however, is problematic; it is rooted in anarcho-capitalistic ideologies that posit the free market as the ultimate purveyor of freedom. In lieu of this, I argue that there is an apparent socio-political oversight regarding the nature of the market as it pertains to the sovereign state, and that those seeking to pass sovereignty to the individual should reject the capitalist institutions that necessitate a coercive state apparatus. Rather than this, there is true emancipatory potential in the development of the digital commons.
\end{abstract}




\section{Acknowledgements}

There are so many people to thank in a process such as completing a thesis. Obviously, I simply would not have been able to do it without my supervisors Aaron Henry and Randall Germain. I don't really feel like I can do justice to how much their guidance during this time means to me, and I sometimes felt like I didn't really deserve the continuous support and belief that they showed in me. In different ways, they were always there when I needed them the most and I cannot thank them enough for guiding me and helping me to find my way.

I was also very blessed to have Donna in my corner, who has been a true friend throughout our time knowing each other and has helped me find my balance in more ways than she can know.

While it's likely standard for one to acknowledge their family here, I would say that I truly would not have been able to do this without mine. Jeff, Alyssa, and Meara; thank you so much for always welcoming me in without hesitation and allowing me to be at home when I was with you. Shaun; there has never been a doubt in my mind that you are incredibly proud of what I am doing and it's so very easy for me to find solace in that when my mind starts wandering. Aunt Marilynn; I just could not have accomplished anything without the love and support you have given me throughout the years - I know that I'm not always the best at showing it, but please know that it means the world to me and it always will. Dad; this is all because of you.

I'd like to also thank Jake and Lisa Scoffield, as well as William and Cassie. When I needed a home so I could get this done, they were there for me - like they've always been there for me. I could not be more grateful to have them in my life and I only hope that I can one day do for them what they have done for me. 
There is also a person who has constantly pushed me to be the best that I can be and has always been there as a beacon of support and motivation in everything that I do. Dave, I would take this opportunity to truly thank you for everything. I wouldn't be here without you and please know that there is not a day that goes by that I don't realize that. I wish nothing but the absolute best for you and Alicia.

There are so many more people that I would acknowledge: Simone, you were the one who brought me back when I was about to give in and I will always cherish that. Chap, Owe, Mully, Drewboy - thanks lads for keeping me sane through everything. I would also say cheers to all those I love from Colborne and from Peterborough. Charlotte, there's nothing I can say here that would do it justice, so I'll tell you next time we have a drink.

It's the hardest thing because I really wish I could recognize everyone who has made an impact on me; but alas, that would be very time consuming. You know who you are. Thank you.

Dedicated to my mom, who still guides my hand after all of these years. 


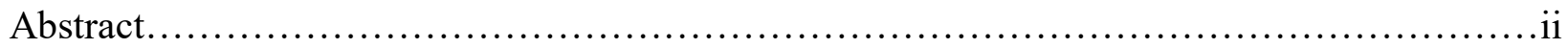

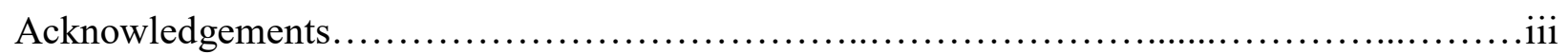

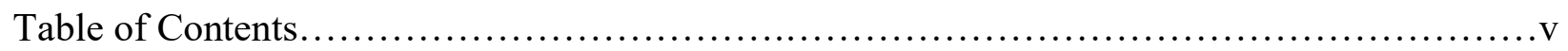

Abbreviations.......................................................................

Introduction..........................................................................1

Preamble: The Landscape of Digital Identity ........................................................5

The Remaking of Identity in the $21^{\text {st }}$ Century...................................

Overlapping Conceptions of Digital Identity...................................11

When Someone Else Owns Your Identity: A Cause for Concern.......................17

Chapter 1: The Self-Sovereign Digital Identity.........................................28

The Potentials of a Self-Sovereign Digital Identity...............................28

The Realizations of a Self-Sovereign Digital Identity................................ 38

Digital Nations and Virtual Worlds..........................................48

Chapter 2: Smart Contracts, Decentralized Networks, and the Commons...................64

The Potentials of Smart Contracts and DAOs....................................64

The Realizations of Smart Contracts and DAOs................................. 74

Networks and the Commons.............................................. 85

Concluding Analysis...................................................................100

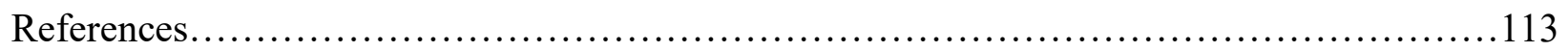




\section{$\underline{\text { Abbreviations }}$}

AI

API

BYOI

CA

CBA

CBPP

CEO

CRF

DAO

DAP

dApp

DBVN

DCN

DIA

DIACC

DLT

eID

ERC-20

EVM

FLOSS

IOS

IOT

KSI

KYC

NSA

PKI

PoS
Artificial Intelligence

Application Program Interfaces

Bring Your Own Identity

Cambridge Analytica

Canadian Banking Association

Commons-Based Peer Production

Chief Executive Officer

China Rapid Finance

Decentralized Autonomous Organization

Decentralized Autonomous Platform

Decentralized Blockchain Application

Decentralized Borderless Voluntary Nation

Digital Content Next

Digital Impact Alliance

Digital ID Authentication Council of Canada

Distributed Ledger Technology

Estonian Digital Identity Card

Ethereum Compliant Tokens

Ethereum Virtual Machine

Free/Libre Open Source Software

Apple Operating System

Internet of Things

Keyless Signature Infrastructure

Know Your Customer

National Security Agency

Public Key Infrastructure

Proof of Stake 


$\begin{array}{ll}\text { PoW } & \text { Proof of Work } \\ \text { RFR } & \text { Refereum Coin } \\ \text { RPG } & \text { Role Playing Game } \\ \text { SBD } & \text { Steem Blockchain Dollar } \\ \text { SCS } & \text { Social Credit System } \\ \text { SIP } & \text { Security Identity Platform } \\ \text { SP } & \text { Steem Power } \\ \text { SPN } & \text { Sapien Coin } \\ \text { SSDI } & \text { Self-Sovereign Digital Identity } \\ \text { SSO } & \text { Single Sign On } \\ \text { TMNT } & \text { Traditional Management Nullification Tool } \\ \text { UNHCR } & \text { United Nations High Commission for Refugees } \\ \text { WIN } & \text { World Identity Network }\end{array}$




\section{Introduction}

In 2008, an anonymous individual going by the codename of Satoshi Nakamoto released the protocol for a technology that has the potential to be one of the most impactful technological development of the 2000s. The title of the white paper that unveiled the technology was "Bitcoin: A Peer-to-Peer Electronic Cash System," and it was developed primarily as a means of providing an electronic payment system based on cryptographic proof rather than being dependent on the trusted third party of financial institutions. ${ }^{1}$ Since its development, Bitcoin has entered into mainstream discussion inspiring both trepidation and awe due to its volatile valuation and perhaps because of its use by criminal networks. Lost in this popular craze, however, is the potential of the underlying technology in blockchain that allows for Bitcoin to operate - though there is certainly significant hype within select circles. Indeed, the technology is recognized by many in the know as revolutionary. If it is to be developed proper, it will without doubt have a monumental impact on an incredible variety of networks, organizations, and institutions; though this is just figuratively scratching the surface of its potential. Indeed, blockchain technology could in fact change the very nature of the socio-political relationships that constitute a society.

This essay will be looking at the revolutionary implications of this technology with a predominant focus on the fields of digital identity and digital organization. My research question is rather simple: how should we conceive of this revolutionary potential? In order to answer this, I will examine how the technology works and what is being done with it in both of these fields. From there, I will provide socio-political theorization on what these developments imply as well as how they challenge long-standing truisms within the liberal world order. This theorization

\footnotetext{
${ }^{1}$ Satoshi Nakamoto, Bitcoin: A Peer-to-Peer Electronic Cash System (2008).
} 
will start off by grounding itself in an analysis and critique of the dominant ideologies generally associated with these decentralized technological developments - that of the 'cypherpunks' and anarcho-capitalism - andI will then use this critique as a sort of springboard for my overall argument that blockchain should be conceptualized as an alternative to the institutions of market capitalism because of its ability to enable the collective governance of the commons.

This essay will be broken up into two chapters and a preamble, with the preamble 'setting the stage' for the coming discussion by focusing on the contemporary landscape of digital identity as it is being developed. In this, there will be no discussion of blockchain as it is meant to more-so serve as an illustration of the trajectory of digital identity without blockchain and why it is concerning. To make this case, the first section of the preamble will look at what is being argued and developed by governments and international institutions such as the United Nations and the World Bank, and the main takeaway is that the world is heading towards legalized digital identities. The second section of the preamble will analyze digital identity under a different pretense, focusing on how corporations such as Facebook and Google create secret digital identities of their users via tracking technology. The final section is titled 'A Cause for Concern' and it is essentially positing that the there are many dystopias that can come to be if these two conceptions of digital identity cross over. In lieu of this, it will be asserted that these issues mostly stem from digital identities being owned and managed by outside forces - which is why the self-sovereign digital identity (SSDI) enabled by blockchain is so important.

The first chapter will thus be looking specifically at what constitutes a SSDI, how it is enabled via the blockchain, and what the more active and popular organizations in the blockchain world are doing with it. In hopes of portraying this properly, the first section of the 
first chapter will thus be fairly technologically-oriented and written with the intent of explaining how the technology works to someone who does not know; blockchain is indeed remarkable, but in order to understand what it is capable of, one must have a basic understanding of how it works. This section gives a simple yet comprehensive overview of Bitcoin and the blockchain ledger and how this technology can be used in the development of SSDI. The following section will then build off of this developed understanding of the technology and proceed to look at who is actually doing what with blockchain as it pertains to SSDI. Further, this section is meant to tease out the evolving nature of citizenship as it intersects with the digital that is very much in development. Finally, once this framework has been built, I will then begin the socio-political theorization in the third section. The main notion I will be critiquing is the idea that these networks will be able to pass sovereignty onto the individual, and in doing this, I will be analyzing the theoretical inspirations of SSDI (stemming from the cypherpunks) and comparing it with the political and philosophical nature of state-sovereignty through the justifications of the social contract. The primary takeaway will be not only is it difficult to see how this aspect of sovereignty could be replicated on a digital identity network, but it is likely not even desirable.

The second chapter will take the concepts that were developed in the first chapter and locate them within the field of digital organization. Consequently, the second chapter will be laid out very similarly to the first being that there is much more to the technology than what was covered in the first. Indeed, the first section of the second chapter will be looking at the capabilities of blockchain enabled by Ethereum having to do with smart contracts, decentralized applications, and decentralized autonomous organizations. Again, this is meant to be written in a simple yet comprehensive manner. The second section of this chapter will also take its inspiration from the first by looking specifically at who is doing what with blockchain 
technology as it pertains to digital organization. In this section I have tried to give as many examples as would be practical to show the scope of Blockchain's impact. Finally, the third section will veer away from the first chapter's format in a sense, but it will continue on with the socio-political theorizations that originated there. In this, I will essentially be continuing on with the analysis and critique of private property as it pertains to the state and showing how it is that blockchain organizations challenge this institution. I will then present a notion of the collective governance of the commons as the strongest conceptualization for challenging the institutions of private property - which I will ultimately posit in the concluding analysis as representing the greatest challenge to the liberal state system; what some readers might recognize as staying true to true anarchism.

It is my sincere hope that this essay is not read, however, as lambasting the organizations I am critical of. Rather, I find them truly inspiring and worthy of immense respect and admiration as they engage in incredible forms of praxis. I am, however, very concerned about the ways in which they are conceptualizing the emancipatory potential of the blockchain. Therefore, I truly hope that this essay can inspire reflection on the underlying (and highly problematic) assumptions of the free market as the purveyor of freedom - there is a far more alluring way to look at it. Further, it is also my hope that this paper can serve as an introduction to the emancipatory potential of blockchain technology to those who do not know. Throughout this paper, I have therefore done my best to keep this flame of potential burning even though I am quite critical of how many seem to be stoking it. And though it is not a primary argument of my essay, I would also say that there is dire lack of the social-sciences in the world of blockchain and it is my belief that this is because not enough people understand just how revolutionary this technology can be. Though we should always be cautious of techno-determinism and the 
thought that a technology can make things better, I do very much hope that this essay is also read as an invitation for those who care to come and help build the world they want to see; because ultimately, the blockchain can serve as an incredibly powerful tool in the creation of a new social, economic, and political order.

\section{Preamble: The Landscape of Digital Identity}

\section{The Remaking of Identity in the $21^{\text {st }}$ Century}

In 2015, the member states of the United Nations agreed upon a set of 17 Sustainable Development Goals to be achieved by 2030. Included in these goals was the $16^{\text {th }}$ goal of Peace, Justice, and Strong Institutions, and the $9^{\text {th }}$ target of this particular goal was 'by 2030, [to] provide legal identity for all, including birth registration.'2 The reason for this is that the World Bank estimates that there are approximately 1.5 billion people in the world who are unable to prove their identity. ${ }^{3}$ Known as the 'identification gap', this is considered to be a significant issue for human rights because the inability to prove one's identity is seen to exclude that individual from rights and services, participation in the formal economy, social security, legal protection, and the ability to achieve a formal education. ${ }^{4}$ This is recognized as going against Article 6 of the Universal Declaration on Human Rights that stipulates 'Everyone has the right to recognition everywhere as a person before the law. ${ }^{5}$

\footnotetext{
2 UN General Assembly. Transforming Our World: the 2030 Agenda for Sustainable Development (A/RES/70/1, 21 October 2015

${ }^{3}$ Estimates by the World Bank ID4D Dataset, as of February 2016. World Bank Group and Center for Global Development. Principles on Identification: For Sustainable Development Toward the Digital Age (February 2017). ${ }^{4}$ Ibid.

${ }^{5}$ UN General Assembly, Universal Declaration of Human Rights (217 A [III], 10 December 1948).
} 
This lack of identity exacerbates the vulnerability of some of the world's most vulnerable people, and at its worst, undocumented individuals - particularly minors - are targeted by human-traffickers and smuggled across borders to be sold into sex brothels, slavery rings, and the human organ trade. ${ }^{6}$ Further, it is estimated by the United Nations that almost half of the world's children under the age of five do not possess a birth certificate, and it is also estimated that there are over 600 million children under the age of 14 without any form of legal identity the majority of whom live in the world's poorest countries. ${ }^{7}$ Such a large number of unidentified people makes it incredibly difficult to target these most-vulnerable populations with progressive policies, and according to the World Bank, "States with weak identification systems have difficulty with government administration, planning, and service delivery, including collecting taxes, targeting social programs, responding to emergencies, disasters and epidemics, managing their borders, and providing security." ${ }^{8}$

Regarding inclusive and sustainable development then, it is logical to see why the United Nations have recognized identity as tantamount to progress. However, achieving a secure legal identity for the world's population may not be possible unless emerging technologies are used to 'leapfrog' the more traditional paper-based systems through the creation of a more 'robust digital-based identity infrastructure' - as recognized by the World Bank and the United Nations. ${ }^{9}$ This is where both organizations have thus directed their efforts, with the United Nations

\footnotetext{
${ }^{6}$ Neil Hughes, “UN Will Use Blockchain and Digital Identities to Combat Child Trafficking” in One World Identity (13 November 2017).

${ }^{7}$ World Identity Network, "World Identity Network and United Nations team up to launch innovative blockchain pilot to help prevent child trafficking" in PRNewswire (10 November 2017).

${ }^{8}$ World Bank Group, Principles on Identification: For Sustainable Development Toward the Digital Age, 4.

${ }^{9}$ World Bank, Technology Landscape for Digital Identification (Washington, DC: World Bank License: Creative Commons Attribution 3.0 IGO [CC BY 3.0 IGO] 2018); Identity 2020 Systems, The Need for Good Digital ID is Universal (2019); World Bank Development Report, Enabling Digital Development: Digital Identity (2016).
} 
fostering ID2020 and the World Bank developing the organization ID4D. ID2020 is an alliance of governments, NGOs, and the private sector founded by Microsoft, The Rockefeller Organization, Accenture, and Gavi. ${ }^{10}$ They seek to provide digital identity to those living without that is 'personal, persistent, private, and portable. ${ }^{11}$ Comparatively, ID4D seeks to help "countries realize the transformational potential of digital identification systems... with global practices and units working on digital development, social protection, health, financial inclusion, governance, gender, and legal issues" 12

This is not to say that the recognition of the usefulness of digital identity is exclusive to international organizations, however, nor is it seen solely as a benefit to international humanitarian goals. Many countries have indeed acknowledged the significant benefits of digital identity and have already begun developing their own versions. The country of Estonia, for example, provides every citizen with a digital identity card (eID) that gives them access to a digital infrastructure where over 600 government services are implemented and where the country's elections take place. ${ }^{13}$ Included benefits are travel within the EU, use as a national health insurance card, proof of identification when logging into bank accounts, to use as an official signature, to check medical records, to submit tax claims, to use e-Prescriptions, for public transit, and so on. In addition, Estonia is the only country in the world where ' $99 \%$ of the public services are available online $24 / 7 .{ }^{14}$

\footnotetext{
${ }^{10}$ Identity 2020 Systems, The Need for Good Digital ID is Universal.

11 Ibid.

12 World Bank Group, Identification for Development (2019).

${ }^{13}$ Access Now, National Digital Identity Programmes: What's Next? (May 2018). 8

${ }^{14}$ E-Estonia. "E-Governance." Available at https://e-estonia.com/solutions/e-governance/ [accessed 17 March 2019].
} 
Though the program is significantly less developed than that of Estonia, In 2008, India engaged in probably the most ambitious project regarding digital identity when it developed 'Aadhaar' ('Foundation') as a means of providing the countries 1.21 billion residents (at the time of development) with a unique 12-digit number linked to their biometrics (iris scan and fingerprints) and demographic data. ${ }^{15}$ An Aadhar number has already been claimed by over 1 billion residents, and in the move to make governing systems digital, the Indian government has also developed DigiLocker which can only be accessed with the 12-digit Aadhar number. ${ }^{16}$ DigiLocker is a key initiative that comes in the form of an App, and it gives each citizen $1 \mathrm{~GB}$ of storage space where they can keep all of their personal documents on a government server. ${ }^{17}$ Citizens can store things like their driver's license and vehicle registrations here so there is no need to carry physical copies - they can just use the Digilocker App (though it is still being developed). ${ }^{18}$ Further, they can also store their education certificates, use it for public transit, be issued a variety of insurances including health through it, and much, much more. ${ }^{19}$

China is also in the process of developing digital identity for its 1.3 billion population by partnering with the country's state-subsidized social media app 'WeChat'. ${ }^{20}$ WeChat already has over a billion daily users and is by far the most-used social media platform in China with nearly 95\% of Chinese netizens utilizing it. ${ }^{21}$ In December of 2017, the Chinese government announced that it would be piloting an ID program through WeChat in the Nansha district of

\footnotetext{
${ }^{15}$ Access Now, Supreme Court of India rules to restrict world's largest digital identity framework (Aadhaar) - but debate continues (26 September 2018).

${ }^{16}$ Ibid.

${ }^{17}$ Government of India, "Benefits of Aadhaar Card" in Vikaspedia (2019).

${ }^{18}$ Government of India, "Digilocker: Your Documents Anytime, Anywhere" in Digilocker (2019).

${ }^{19}$ Ibid.

${ }^{20}$ Zhang Dangyang, "Guangzhou Issues the first Wechat Identification Card, Expected to Launch Nationwide in coming January" in Zinghua (26 December 2017).

${ }^{21}$ Mansoor Iqbal, "WeChat Revenue and Usage Statistics (2019)" in Business of Apps (27 February 2019).
} 
Guangzhou that is home to some 13.5 million. ${ }^{22}$ Expected to be fully implemented throughout China by 2019, the project allows individuals to link their national ID card with their WeChat account by verifying themselves with facial recognition. ${ }^{23}$ Individuals can then use the WeChat 'web certificate' in place of their national ID card for government services, accommodations, bus/train tickets, and so on. ${ }^{24}$ This is in addition to the app's more traditional use as a social media application where users can send each other messages through a variety of formats (text, picture, voice, video, etc.) while also sharing posts to their friend space that others can 'like'. ${ }^{25}$

In July of 2016, the European Parliament and The Council of the European Union passed regulation No. 910/2014 which was the first formal step towards establishing a trusted interoperability of digital national identities within Europe. ${ }^{26}$ This would allow the digital identity of one member state to be used to access public services of any other member state. ${ }^{27}$ Rather than universal development, the key to this concept is to set a standard upon which nations within the European Union can develop their own versions of a digital identity that meet an underlying criteria used by all. ${ }^{28}$ Across the ocean in America, companies such as Visa, the Bank of America, JP Morgan Chase, Symantec, and others have formed the organization The Better Identity Coalition to insist that the United States "Prioritize the development of nextgeneration remote identity proofing and verification systems." 29 Though America as a country

\footnotetext{
22 Ibid.

23 Ibid.

24 Ibid.

${ }^{25}$ Ge Wang, Wei Zhang, and Runxi Zeng, "WeChat use intensity and social support: The moderating effect of motivators for WeChat use" in Computers in Human Behavior 91 (2019).

${ }^{26}$ The European Parliament and the Council of the European Union, "On electronic identification and trust services for electronic transactions in the internal market and repealing Directive 1999/93/EC" in Official Journal of the European Union (257/73, 28 August 2014).]

27 Ibid.

28 Jens Bender, eIDAS Regulation: elD - Opportunities and Risks (January 2015).

${ }^{29}$ The Better Identity Coalition, Better Identity in America: A Blueprint for Policymakers (July 2018). 2
} 
might be a little late to the game, they might have metaphorically jumped the line when American companies Microsoft and Mastercard announced in December 2018 that they are collaborating to create a universally recognized digital identity. ${ }^{30}$ Apple has also filed patents suggesting they would like to be in charge of digital identity management. ${ }^{31}$

North of the American border, the Canadian Banking Association (CBA) is calling for a federated approach to digital identity so that Canada can become a global leader in this arena. ${ }^{32}$ Further, the Digital ID Authentication Council of Canada (DIACC) - a non-profit coalition of the Canadian Government and leading organizations from the private sector - have developed 10 guiding principles for a national Canadian digital identity while also recommending that Canada join Estonia, Sweden, and Luxemburg as digital leaders. ${ }^{33}$ The desire to go digital, according to the CBA, has to do with the expedition of government services, significant cost savings, fraud reduction, improved regulatory compliance, privacy enhancement, being future ready, and things along this nature. ${ }^{34}$ The DIACC further claims that with the vast majority of business moving deeper into the online world, both the public and the private sectors of Canada would benefit greatly from having trustworthy verifiability of one's identity. ${ }^{35}$ The board of directors of this

\footnotetext{
${ }^{30}$ Mastercard Press Release, Mastercard, Microsoft Join Forces to Advance Digital Identity Initiatives (3 December 2018); Windows Central, Microsoft and Mastercard Want to Give You a Universal Digital Identity: One Digital Identity to Rule Them All (3 December 2018).

${ }^{31}$ Malcolm Owen, "Apple wants iPhone to be proof of identity and replace passports" in Apple Insider (9 August 2018).

${ }^{32}$ Canadian Banking Association. Canada's Digital ID Future - A Federated Approach. 2018, available at https://cba.ca/Assets/CBA/Documents/Files/Article\%20Category/PDF/paper-2018-embracing-digital-id-in-canadaen.pdf [accessed 17 March 2019]

${ }^{33}$ Digital Identity \& Authentication Council of Canada, Digital Identity Innovation - Canada's Opportunity to Lead the World (4 August 2017).

${ }^{34}$ Canadian Banking Association, Canada's Digital ID Future.

35 Digital Identity \& Authentication Council of Canada, The Economic Impact of Digital Identity in Canada: Understanding the potential for considerable economic benefits and the cost of inaction (2018).
} 
coalition include CIBC, TD Bank, Bank of Montreal, Dejardins, Interac, PWC, the Government of Canada, and others. ${ }^{36}$

The accumulation of all of these individual efforts has effectively pushed the concept of digital identity to the forefront of contemporary notions of citizenship around the world. In fact, just the examples provided represent nearly half of the world's population and makes the developments intrinsically momentous as representations of societies increasingly intertwining with the digital - something that should be considered a global phenomenon. With that being said though, while all of these countries and international institutions are fostering the transition to a new sort of world where legal identities exist on digital networks, it is unclear how this new world of digital identities will interact not just with other networks within its own framework, but with an old digital world where users have already been issued forms of digital identity how will a legal identity intersect with a Facebook account, for instance? Further, an underappreciated problem to be identified is if the new world and the old world do intersect, who will ultimately manage these networks of identity? As the following section will show, private corporations have been creating incredibly comprehensive digital identities for some time. Who will be in charge when these corporate-owned identities meet with these newly emerging stateowned identities? And further - as the concluding section of the preamble will discuss - should this intersection serve as a cause for concern?

\section{Overlapping Conceptions of Digital Identity}

"One of the errors that the Internet made a long time ago is that there was not an accurate and non-revocable identity-management service... and the best example of an identity-management service today that's reasonably reliable is Facebook"

- Eric Schmidt, CEO of Google 2011.

\footnotetext{
${ }^{36}$ Digital Identity \& Authentication Council of Canada, Membership (2019).
} 
The examples of digital identity provided in the previous section are essentially operating under a more traditional understanding of identity in that an identity is something that is issued to an individual via the state - though the international institutions of the United Nations and the World Bank certainly represents a new twist on this. Nevertheless, identity under this pretext is simply a verifiable representation of who you are, and traditionally this can be found in documents such as a birth certificate, citizenship, SIN number, driver's license, or a passport, as well as documentations such as medical history, certificates, degrees, licenses, and so on. To conceptualize a digital identity under this context, then, would thus be having these sorts of identifying markers on a digital network and being able to verifiably present them in a digital environment - such as is the case with Estonia.

There is a world of digital identities that already exists, however, that is far different than this more traditional understanding. Indeed, users of the internet have long been able to create digital representations of 'who they are' in the form of profiles through a vast variety of different digital mediums; the most obvious example of this in the west would be Facebook, but other popular examples include Google, Twitter, Reddit, LinkedIn, YouTube, Instagram, and so on and so forth (the examples are effectively endless). Additionally, even if it is not explicitly a profile, companies such as Amazon facilitate the creation of an account that tracks all of an individual's shopping history on the site while also allowing that individual to provide a review or rating of their purchase. Amazon again is just the largest and most quintessential example of this, there are thousands if not millions of sites that facilitate the creation of some sort of account that resembles that of an identity - not to mention the creation of characters and avatars in roleplaying games (RPGs) such as World of Warcraft and virtual realities such as Second Life. 
While all of these companies have different primary purposes (and represent an incredibly small proportion of the possible examples), they all operate under the same basic tenets in that they allow a user to create a digital identity on the company's network by providing their information and identifying markers. The user is then given primary access and is able to manage and control certain aspects of their profile as they navigate whatever it is that the network provides; though the control that the user is provided is quite limited in many respects. Indeed, while the networks may employ the façade that users own their accounts, the reality is that users more-so just have access to accounts that are owned by the network. Facebook, for instance, claim in their principles that 'People should own their information...People should have the freedom to decide with whom they will share their information, and to set privacy controls to protect those choices. ${ }^{37}$ In reality, however, there is much more to a Facebook account than the information that the user uploads.

The digital identity facilitated through Facebook can indeed include most things a user does online. A primary example of this is that Facebook also effectively manages many of the other accounts that a user creates on alternative networks being that many users are simply unable to manage all of their different profiles; the organization Dashlane, for instance, estimates the average American internet user has 130 accounts registered to them. ${ }^{38}$ In lieu of this, the term 'password fatigue' has emerged to describe the overbearing nature of so many accounts, and this has led to the emergence of a concept referred to as 'Bring Your Own Identity' (BYOI) as a sort of federated identity management system. ${ }^{39}$ What BYOI does is create a network of interoperability amongst individual networks so that one identity (account or profile) can be used

\footnotetext{
${ }^{37}$ Facebook, Facebook Principles (2019), available at https://www.facebook.com/principles.php.

38 Tom Le Bras, "Online Overload - It's Worse Than You Think" in Dashlane (21 July 2015).

39 RSA, Bring-Your-Own-Identity Gains Steam in Information Security (13 April 2016).
} 
as a single-sign on ( $\mathrm{SSO}$ ) to access third-party platforms; a common example of this would be using a Facebook profile or a Google account to sign up for an alternative service such as a news subscription or an online dating platform. In terms of the most popular 'gatekeepers' of SSO authentication, Google and Facebook accounted for $81 \%$ of social login preference according to a study conducted by Janrain in 2014 , with Facebook in the lead at $44 \%{ }^{40}$

While BYOI and SSO certainly facilitate far greater convenience than having separate accounts for each individual site, it simultaneously empowers the organizations hosting the SSO to act as the figurative arbiters of digital identity across the web. This empowerment has left some organizations wary; the DIACC, for instance, states “...it is not clear that all users understand the implications of using such a login service. For example, what data is shared between the services and Facebook? Where else, and how could a user's activities be shared?"41 In Facebook's data policy, Facebook clearly states that they track a user's activities across different networks through their partners: "These partners provide information about your activities off Facebook —including information about your device, websites you visit, purchases you make, the ads you see, and how you use their services - whether or not you have a Facebook account or are logged into Facebook." ${ }^{42}$ The digital identity being managed by Facebook, then, is not solely the details or pictures an individual uploads to their public profile, but rather, it includes a private profile accessible only to Facebook which includes everything they are doing via the SSO. Further, Facebook's application program interfaces (APIs) are littered all over the

\footnotetext{
${ }^{40}$ Barry Levine, "What's the Most Popular Social Login on B2B Sites?" in CMS Wire (15 July 2014).

${ }^{41}$ Digital Identity \& Authentication Coalition of Canada, Building Canada's Digital Identity Future (May 2015).]

42 Facebook, Data Policy (19 April 2018), available at https://www.facebook.com/about/privacy/.
} 
web in the form of 'like' and 'share' buttons, so whenever a user clicks one of these, more data regarding the user is sent to Facebook's private version of the individual's digital identity. ${ }^{43}$

Facebook is certainly not alone in creating privately-managed digital profiles of its users, and it could be said that it might not even be the most sinister. Indeed, Google provides the world's most popular web browser, search engine, and mobile platform, thus simultaneously vaulting it into the role of the world's most popular digital advertising company. ${ }^{44}$ Primarily through the Android operating system and the Chrome browser, Google is able to collect significant data on its users even in the absence of any user interaction; research conducted by the organization Digital Content Next (DCN) showed that “.... dormant, stationary Android phone (with Chrome active in the background) communicated location information to Google 340 times during a 24-hour period, or at an average of 14 data communications per hour." ${ }^{45}$ Once the user starts interacting with the phone, these communications increase significantly (even if the user is not using popular Google apps such as Youtube, Gmail, Google Maps, Google Search, etc.). ${ }^{46}$ Further, Google can collect most of the user's personal information through their usage of apps on Android (similar in the way that an SSO does) whilst simultaneously monitoring their purchases, locations visited, websites browsed, mobile activity, and various other device related identifiers. ${ }^{47}$ In all, a user's very interaction on the google interface is summarized in the form of a digital representation which the DCN showed to be remarkably accurate. ${ }^{48}$

\footnotetext{
${ }^{43} \mathrm{Ibid}$.

${ }^{44}$ Douglas Schmidt, "Google Data Collection" in Digital Content Next (August 2018). 2

${ }^{45}$ Ibid., 3

${ }^{46} \mathrm{Ibid}$.

${ }^{47}$ Ibid.

${ }^{48} \mathrm{Ibid}$.
} 
The examples of Facebook and Google serve as representations of what E.N. Beck refers to as The Invisible Digital Identity, though the author is quick to note that surveillance technologies long predate Facebook and Google. ${ }^{49}$ The technologies Beck is primarily referring to are 'cookies' and they were first released with Netscape's Navigator 1.1 in $1994 .{ }^{50}$ Cookies are essentially a small file sent to a computer's hard drive automatically when a user visits a website. The cookie then gathers information about other websites a user is browsing and sends this information back to the original website. There are a variety of reasons why companies use cookies (many of which are either mundane or rather useful), but many cookies released should simply be considered as inauspicious. Tracking cookies, for instance, can be placed on a user's computer or device that follow the user around the web and can be accessed by websites that did not create them; this allows a cookie's creator to receive data about the user whenever the user is on a site with a resource belonging to the creator. This means that the aforementioned API's created by Facebook in the form of 'like' or 'share' do not even need to be clicked for the website to be sharing information regarding what you did on that website with Facebook. ${ }^{51}$

This sort of 'invisible identity' enabled by passive tracking in the form of BYOI, SSO, and cookies allows for these private corporations to create incredibly comprehensive identities of its users - the likes which we only have a basic idea of, but it is safe to say that they effectively encompass most things an individual does online. And while it can be said that this massive amount of data being collected by companies such as Facebook and Google is coming more and more into public and popular debate, most should find it incredibly concerning that not only do

\footnotetext{
${ }^{49}$ Estee Beck, "The Invisible Digital Identity: Assemblages in Digital Networks" in Computers and Compositions (35, March 2015). 129

$50 \mathrm{lbid}$.

${ }^{51}$ Facebook. Cookies \& Other Storage Technologies (4 April 2018); Belgian Privacy Commission, Facebook Tracking Through Social Plug-ins (27 March 2015).
} 
we not know what these corporations are doing with these digital identities, but it is not clear how they will intersect with the legal identities that are being developed. One thing that does seem to be clear, however, is that if things do not change drastically, there will be such an intersection; the precedence has seemingly already been set being that Facebook has run a trial asking Indians to provide their Aadhar number in order to create a new account (though Facebook later claimed that nobody would be forced to use their number). ${ }^{52}$ Still, a somewhat contentious relationship exists being that users of Digilocker in India can login to the application with their Facebook ID validation. ${ }^{53}$ And just to further stoke the hypothetical fire, in late 2018 Facebook acquired the company Confirm.io which specializes in verifying government-issued identification cards as authentic. ${ }^{54}$

\section{When Someone Else Owns Your Identity: A Cause for Concern}

"The old world is dying, and the new world struggles to be born: this is the time of monsters." - Antonio Gramsci

Regarding this intersection, then; though the comparison is certainly imperfect (and some might suggest far-fetched), the example of digital identity facilitated trough WeChat in China highlights some of the primary concerns regarding the intersection of a user's interaction on digital networks with that of an 'official' identity. WeChat is owned by the Chinese internet giant Tencent, which is claimed to be the world's largest social media company after it surpassed Facebook's value in $2017 .{ }^{55}$ Tencent also partners with the organization China Rapid Finance (CRF) which has been given a license to develop what the Chinese government has been

\footnotetext{
52 Jagmeet Singh, “Facebook Confirms Aadhaar Prompt for New Users, Says It Isn't Necessary” in Gadgets 360 (17 December 2017.

${ }^{53}$ Digilocker National e-Governance Division, Digilocker User Manual, 8.

54 Josh Constine, "Facebook Acquires Biometric ID Verification Startup Confirm.io" in TechCrunch (2018).

55 James Titcombe, "China's biggest social media company Tencent is now worth more than Facebook" in The

Telegraph (21 November 2017).
} 
referring to as the Social Credit System (SCS). ${ }^{56}$ In addition to CRF and Tencent, the other most prominent developer of SCS is the Ant Financial Services Group which is an affiliate company of Alibaba, calling their project Sesame Credit. ${ }^{57}$

Alibaba is sometimes compared as a Chinese equivalent to Amazon even though its online sales and profits have surpassed all US retailers combined since 2015 - including Amazon, Walmart, and Ebay. ${ }^{58}$ One of the primary services of Alibaba beyond internet purchases is AliPay, which has over 500 million monthly active users and can be used for everything from "restaurants, taxis, school fees, cinema tickets and even to transfer money to each other." 59 In addition to these two super-giants, Sesame Credit has also teamed up with China's most popular ride-hailing company ('China's Uber equivalent') in Didi Chuxing, as well as the country's largest online dating service in Baihe. ${ }^{60}$ Between just these companies alone, it is not hard to imagine - though nearly impossible to fathom - how much data is being collected about the officialized digital identities of China's citizens.

Where the cause for concern comes in is what the Chinese government is doing with this data in its social credit program. Popularized in the West by the Netflix hit show Black Mirror in an episode released on October $21^{\text {st }}, 2016$ called Nosedive, the concept of SCS is the rating and ranking of individuals based on their actions and interactions. ${ }^{61}$ While the show is broadly considered to be a dystopian portrayal of technology gone awry, what they depicted is not so

\footnotetext{
${ }^{56}$ Rachel Botsman, Who Can You Trust: How Technology Brought Us Together and Why It Might Drive Us Apart (New York: Public Affair Books, 2017): 161.

57 Ibid., 163

${ }^{58}$ Allen Cheng, "Alibaba vs. The World" in Institutional Investor (25 July 2017).

59 Botsman, Who Can You Trust, 173

60 Ibid.

61 Ibid., 163
} 
different from what is being developed by the Chinese government. On June $14^{\text {th }}, 2014$, China published a document called the 'Planning Outline for the Construction of a Social Credit System' which outlines the blueprints for SCS. ${ }^{62}$ In the words of the plan, "Big data will become the most important and powerful driver to accelerate the modernization of governmental governance capacity." ${ }^{63}$

The SCS is an amalgamation of all the 'relevant data' compiled by these companies, and then a 'complex algorithm' (in the words of Sesame Credit) is used to sort this data and give the user a score between 350 and $950 .{ }^{64}$ This score is then used to determine a user's social worth, with a high score seen as a status symbol allowing them to take out loans, rent cars without a deposit, faster check-in at hotels, fast-tracked for visa's, and so on. ${ }^{65}$ To have a bad score, however, will result in slower internet, restricted access to more desirable entities such as restaurants, ability to be hired by certain places including civil services, access to certain school, and so on. ${ }^{66}$ According to the planning outline, "If trust is broken in one place, restrictions are imposed everywhere." ${ }^{67}$ As an example of the scope of these restrictions, in 2018, it was reported by The Guardian (referencing China's National Public Credit Information Centre) that 17.5 million flights had been cancelled due to citizens being on the social credit black list. ${ }^{68}$

Vital to know in SCS, however, is that the actual algorithms used to determine one's social credit rating are kept secret. Being that the algorithms are kept secret, it is impossible to

\footnotetext{
${ }^{62}$ Rogier Creemers, "China's Social Credit System: An Evolving Practice of Control" in SSRN (9 May 2018). 13

${ }^{63}$ Cited from Botsman, Who Can You Trust, 163

${ }^{64}$ Ibid., 163-165

${ }^{65}$ Ibid., 167

${ }^{66}$ Ibid., 172

${ }^{67}$ Creemers, China's Social Credit System, 13

${ }^{68}$ Lily Kuo, "China bans $23 \mathrm{~m}$ from buying travel tickets as part of 'social credit' system" in The Guardien (1 March 2019).
} 
know what is being taken into consideration. What is speculatively known, however, is how much data companies like Tencent are collecting, and again we can easily imagine (even if it is impossible to fully fathom) what kind of invisible digital identities are being created - though perhaps the concept of an invisible digital identity needs to be updated. What can be seen in China could be considered as an evolution of the invisible identities created by the unknown collection of data via tech giants amalgamating with officialized identities of its citizens in what Dr. Rogier Creemers, who specializes in Chinese Law and Governance, refers to as an Orwellian nightmare: "an omniscient machine hoovering up the massive amounts of data individuals generate as they plod through their lives, processing it to deliver a quantified score that creates an ideological and consumerist straightjacket for every Chinese citizen." ${ }^{99}$

The invisible identities created by the tech giants of China are now simply not so invisible, and the Chinese government is able to manipulate them to acquiesce its population. The question to be posed, then, is it really so unconceivable to suggest that this could occur elsewhere? Though there is without any doubt a significant difference between Chinese regulation of the internet (not to mention direct entanglement between the government and the tech giants) compared to liberal democracies that almost goes without saying, Rachel Botsman makes the prescient case that SCS in China is still worthy of comparison to the tech giants found in liberal democracies. ${ }^{70}$

The comparison has to do with the invisible/not-so-invisible identities created and managed by the unknown collection of data and what is done with it. In this, though many have seen allegory between SCS in China and the infamous Nosedive episode of Black Mirror that

\footnotetext{
${ }^{69}$ Creemers, China's Social Credit System, 26
}

70 Botsman, Who Can You Trust, 174-189 
shows the downfall of main character Lacie as her social credit score starts to plummet, the creator of the show, Charlie Brooker, said in an interview with Rolling Stone that his inspiration for the episode actually came from taking Uber where customers and drivers can rate each other. $^{71}$ In this, rating is indeed a very popular concept in Western societies, and other rating services include Yelp, Airbnb, RateMyProfessor.com, and so on. Further, errand runners and gig workers like Taskrabbit and Deliveroo are rated; Fitbit gives you a fitness score; Klout scores are given based on individual's social media influence; Datecheck gives an instant background check on potential dates; Goodreads and Amazon provide author ratings; the list goes on and on. $^{72}$

There even exists a (thankfully) near-universally lambasted app called Peeple, where users could give each other a rating between 1 and 5 based on their own subjective reasoning - a sort of 'Yelp for people' almost identical to the episode Nosedive (though in response to heavy criticism the app has since changed).$^{73}$ All of these apps collect data about its users and it is unknown what is done with this data or who it is shared with. However, it is safe to assume that much of this data is amalgamated in some central server based on cookies, the fact that they exist on the android operating system, and that many of the organizations (including Peeple) are managed by the SSO of Facebook. What China has done is amalgamate this sort of data into a single score based on its direct entanglement with the tech giants and its regulation of the internet - what Creemers describes as "where Big Brother and big data conspire to finally realize the totalitarian impulses of China's autocratic leaders." ${ }^{24}$ While it is safe to say that the totalitarian

\footnotetext{
${ }^{71}$ David Fear, “'Black Mirror': Inside New Season of Chilling Cult U.K. Horror Series" in Rolling Stone (13 October 2016).

72 Botsman, Who Can You Trust, 182

73 Ibid., 177

${ }^{74}$ Creemers, China's Social Credit System, 2-3
} 
impulses of liberal-democracies are at the very least less-present, it should still serve as a cause for concern that all of this data is being collected and amalgamated by unaccountable and inaccessible centralized parties.

With all of this being said, however, there is still incredible differences between the People's Republic of China and that of liberal democracies in the West that again almost goes without saying; one of the more predominant ones in this particular discussion being China's direct access to the data collected by Chinese companies. Indeed, on June 1, 2017, a law came into place that mandated businesses managing their own data networks must give access to Chinese security officials upon request (including technical support). ${ }^{75}$ To continue on with the comparison, however - even if it is most-definitely imperfect - what was revealed by the National Security Agency (NSA) files of America in 2013 is that the American government also has the ability to access the metadata of massive corporations such as Google, Microsoft, Apple, Yahoo, and Facebook, allowing it to create a detailed profile deemed a 'pattern of life' for a given target. ${ }^{76}$

Though the corporations have each vehemently denied giving open access to the government short of a court order ${ }^{77}$ the PRISM project of the NSA has been clouded in secrecy regarding how it pulls metadata from service providers, what exactly it is pulling, and what it is doing with it; it begs the question is a pattern of life not somewhat analogous to a social credit rating? Still, it should be noted that many of the most prominent corporations have pushed back

\footnotetext{
${ }^{75}$ Elizabeth Economy, The Third Revolution: Xi Jinping and the New Chinese State (England: Oxford University Press, 3 May 2018). 74

${ }^{76}$ Ewen Macaskill \& Gabriel Dance, "NSA Files Decoded: What the Revelations Mean For You" in The Guardian (1 November 2013).

${ }^{77}$ Chris Welsch, "Apple, Google, Microsoft, Facebook, Yahoo, and more deny providing direct access to PRISM surveillance program" in The Verge (6 June 2013).
} 
against the spying, and even Glenn Greenwald, the journalist who broke the story based on whistle-blower Edward Snowden's leaks, stated "These companies are now engaged in a genuine commitment to demonstrate that they're willing to protect privacy even against the US government." ${ }^{178}$ It is also worth noting that Tencent scored a 0/100 on its encryption levels based on a study conducted by Amnesty International, where Facebook scored 73/100; Apple scored 67/100; Google scored 53/100; and Microsoft scored 40/100. ${ }^{79}$

Nevertheless, though the amount of access to metadata that Western governments have is unknown (as is the extent of the profile they are creating for individuals), what is known is that the digital identities being created by these massive corporations are done without the knowledge or input from the people for whom they are being created. Further, they are predominantly done under the profit motive of creating and packaging up the digital representation of individuals and selling it for targeted advertising; as per the decades old adage popularized and modified by CEO of Apple Tim Cook, "When an online service is free, you're not the customer. You're the product." ${ }^{80}$ The American election of 2016 and the Brexit vote of 2016 portrayed what can go amiss in a liberal democracy when people's digital identities are being sold as a product.

In 2010, researcher of psychometrics Michal Kosinski found that a user's participation on the Facebook network - predominantly with what they 'like,' post, and share - could be algorithmically accumulated to produce a remarkably accurate portrayal of the individual. ${ }^{81}$ Amalgamizing the data could show a user's likelihood of being Republican or Democrat, single

\footnotetext{
78 Laura Hautala, "The Snowden effect: Privacy is good for business" in CNET (3 June 2016).

${ }^{79}$ Amnesty International, For Your Eyes Only (2016).

${ }^{80}$ Sam Colt, "Tim Cook Has an Open Letter to all Customers that explains how Apple's Privacy Features Work" in Business Insider (17 September 2014).

${ }^{81}$ David Sumpter, Outnumbered: From Facebook and Google to Fake News and Filter-bubbles - The Algorithms That Control Our Lives (Bloomsbury: Bloomsbury Publishing, 2018). Chapter 4
} 
or in a relationship, sexual orientation, and so on. ${ }^{82}$ The data could also be used to determine things like IQ level and race. Finally, it could even be used to predict the most universal 'Big Five' psychological components of personality: openness, conscientiousness, extroversion, agreeableness, and neuroticism. ${ }^{83}$ Knowing this data and these personality traits proves to be remarkably valuable in influencing an individual, and though Kosinski vows that he had no part in it, similar methods were allegedly used by the company Cambridge Analytica (CA) to determine what kind of advertising would have the greatest effect on individuals during campaigns such as the American Election and the Brexit vote - though CA has since denied that they used these techniques and many experts in the field have doubted that the algorithms could even be this sophisticated, attributing the claims to 'hyperbole' ${ }^{84}$

Regardless of whether or not the capabilities of firms like CA are exaggerated, the fact of the matter is that the public simply does not know what is being collected about them, by whom, or what is being done with it (who it is being sold to) - though we do know that CA was able to gain access to at least 87 million Facebook users, bragged about using the Big Five personality model prior to being scrutinized publicly about it, targeted individuals with personalized advertising, claimed itself as instrumental in Donald Trump's victory, and had the former chief strategist of Trump's white-house Steve Bannon as a board member. ${ }^{85}$ In one of the most prominent accounts of the CA scandal titled "The great British Brexit robbery: how our democracy was hijacked" in The Guardian newspaper, author Carol Cadwalldr claims "Data... is

\footnotetext{
82 Ibid.

$83 \mathrm{lbid}$.

${ }^{84} \mathrm{Ibid}$.

${ }^{85}$ Ibid., Chapter 5
} 
being silently amassed, harvested and stored. Whoever owns this data owns the future." 86 In China, the owner of the data is using it to create an autocratic 'Orwellian nightmare' the likes of which the world has never really seen. In America and Britain, it has been auctioned off and used to 'hijack' democracy; not to mention the countless other countries and instances where it has been used to game the people. Finally, it is not hard for one to let their imagination roam to see the infinite other dystopian-like situations in which it could be used.

Being that the world is seemingly moving towards officialized digital identities, it is essential that discussions regarding who owns these digital identities are at the forefront of any developments, as well as what they are doing with them. As Cadwalldr poetically pointed out, whoever owns these identities owns the future - which makes it fairly easy to see why organizations such as Microsoft, Mastercard, The Rockefeller Organization, Accenture, Gavi, Visa, The Bank of America, JP Morgan Chase, Symantec, the CBA, CIBC, Interac, and Apple have all tried to claim their stake. Further, being that most already have some form of invisible digital identity, knowing how these invisible digital identities will intersect with officialized identities is beyond critical; how will that data collected from your Facebook profile coexist with your national identity? If the two are linked, will what you 'like' affect your credit score? Will Google have access to your sin number if you use an Android phone for your identification? Will Apple? Facebook and Twitter have a very legitimate fake user problem where fake bots are alleged to have swayed the opinions of people; in order to alleviate this issue, will they require something like an Aadhar number in order to use their services? If so, will they be able to see the details of that number and know where you live? Will Cambridge Analytica be able to

\footnotetext{
${ }^{86}$ Carol Cadwalldr, "The great British Brexit robbery: how our democracy was hijacked" in The Guardian (7 May
} 2017). 
identify you as susceptible to a certain political message and come knock on your door with a pamphlet for the alt-right?

Related to CA coming to knock on your door, the digital human rights organization Access Now have also correctly identified significant potentials for human rights violations centered around a centralized digital identity database based on biometrics - such as is the case with most organizations attempting to develop it today. Not only is a database like this susceptible to breeches by malicious actors, but it is by its very nature a "pervasive means of identification, tracking, or control." ${ }^{87}$ At its worst, an illustrious account conducted by Elise Thomas for Wired Magazine showed how the United Nations High Commission for Refugees (UNHCR) was not only collecting biometric data on the Rohingya refugees fleeing genocide in Myanmar (which is often done without informed consent according to a UN internal audit from 2016), but was sharing this data with the Bangladeshi government (the host of the majority of the refugees) who at the time was in negotiation with Myanmar to send the refugees back - despite overwhelming signs that Myanmar was continuing with its ethnic cleansing: what UN Special Envoy on Human Rights Yanghee Lee described as 'the hallmarks of a genocide' ${ }^{88}$ Further, the data that was being collected was continuously built upon; according to UNHCR spokesperson Cécile Pouilly, "With each contact, including protection intervention, document renewal, assistance delivery, interview to determine refugee status and assessment of solutions, UNHCR builds the identity data held, and cross-checks and confirms previous elements...UNHCR often has detailed knowledge of given individuals spanning years or decades, and with biometrics,

\footnotetext{
${ }^{87}$ Access Now, National Digital Identity Programs: What's Next? (May 2018). 2

${ }^{88}$ Elise Thomas, "Tagged, tracked and in danger: how the Rohingya got caught in the UN's risky biometric database" in Wired (12 March 2018).
} 
those identities can be recognized across any UNHCR location." 89 A rhetorical question to be posed here is what could possibly go wrong when all of the intimate data of a collection of individuals - tied to their biometric identifiers so they could not possibly 'not-be identified' - is being shared with a government that has explicitly stated that it does not want the people there and is in negotiation with a country that has attempted genocide against them?

While the example of the Rohingya is certainly as dire a representation as could be presented, it is still emblematic of the problems associated with the centralized collection of digital identity; as is the case of SCR in China and as is the case of Cambridge Analytica in the United States and Britain. To put it simply, and in the words of a highly-referenced blog post by Christopher Allen titled The Path to Self-Sovereign Identity: "Centralization innately gives power to the centralized entities, not to the users." 90 What should be apparent based on this discussion is there is a lot to be concerned about when unaccountable centralized institutions have the power and are in control of individuals' identities. Being that legalized forms of digital identity are on the horizon, however, there is a small serendipity to be found in the development of a technology that enables an alternative to what is being developed; and in this, it is possible that power will be given back to user.

\footnotetext{
${ }^{89} \mathrm{lbid}$.

${ }^{90}$ Christopher Allen [Blog], The Path to Self-Sovereign Identity (25 April 2016).
} 


\section{Chapter 1 - The Self-Sovereign Identity}

"Another world is not only possible, she is on her way. On a quiet day, I can hear her breathing."

- Arundhi Roy

\section{The Potentials of a Self-Sovereign Digital Identity}

Amid all of the discussions of digital identity and the issues of centralized control, many have indeed turned to the most prominent technological development that allows for digital decentralization - that of blockchain technology. And though blockchain has turned into a bit of a utopian catchphrase in which it is seen to be the (perhaps misguided) answer to every political and sociological issue, the technology in itself does allow for incredible evolutions in a vast variety of different fields; including finance, governing, the internet of things (IOT), voting, registrars; and of course, identity.

Brought to the world with the pseudonymous release of Bitcoin in 2008, blockchain is the underlying technology that allows for individuals to transfer value to each other without the need of a 'trusted third party.' 91 What the pseudonym Satoshi Nakamoto provided in the release of the Bitcoin White Paper was an 'electronic payment system based on cryptographic proof' that did not need a financial institution to verify and authenticate the transfer of funds, but rather, every node on the network would confirm the transaction and stamp the seal of approval. ${ }^{92}$ This collection of stamps would then become a 'block' to be added to irreversible history of collected blocks, creating a 'chain.'

This ‘block-chain' would then serve as an irrefutable and immutable ledger for all transactions, creating a trusted and verifiable method for record-keeping that was distributed

\footnotetext{
${ }^{91}$ Satoshi Nakamoto, Bitcoin: A Peer-to-Peer Electronic Cash System (2008).

92 Ibid.
} 
amongst the nodes that made up the network. Most importantly and revolutionary, however, is that there is no centralized control; every node on the network has a complete copy of the entire database. ${ }^{93}$ Control is thus held by all of the members and none of the members, and nobody can alter the data because it is fully accessible to all the nodes who would detect and reject the misdoing. For any data to be added to the base, all of the nodes must come to a consensus and agree to have it added - which is done through a complex algorithmic protocol called Proof of Work (PoW) on the Bitcoin network (though other concepts exist). The mechanism for achieving consensus in PoW financially incentivizes each node to maintain the network in a process called 'mining' - but this will be covered in far more detail in the following chapter.

With that being said, this particular distributed ledger technology (DLT) in Bitcoin allowed for individuals to transfer 'money' - value in the form of bitcoins - across the world in a trusted and verifiable manner without the need for any financial institution. Though Bitcoin gained infamy and notoriety for its use by criminal networks, the fact that criminals could place their trust in a set of code and algorithms for the transfer of the equivalent to millions of American dollars seemingly proves the authenticity of this newly developed currency as an alternative to nationally regulated fiat currencies monitored through the 'trusted third party' of financial institutions.

While Bitcoin was the first example of DLT, the transfer of value is most definitely not the only application for which blockchain or DLT can be used. Indeed, many have recognized the usefulness of a decentralized and distributed ledger for a vast and eclectic variety of different needs, and thousands of different blockchains and DLTs have emerged to address them.

${ }_{93}^{93}$ Quinn Dupont \& Bill Maurer, "Ledgers and Law in The Blockchain" in Kings Review (23 June 2015). 
According to Melanie Swan, a pioneer in envisioning the future of blockchain, “...the coordination, record keeping, and irrevocability of transactions using blockchain technology are features that could be as fundamental for forward progress in society as the Magna Carta or the Rosetta Stone. In this case, the blockchain can serve as the public records repository for whole societies, including the registry of all documents, events, identities, and assets." ${ }^{\prime 94}$

Further, in the words of Don and Alex Tapscott, the blockchain "can be programmed to record not just financial transactions but virtually everything of value and importance to humankind: birth and death certificates, marriage licenses, deeds and titles of ownership, educational degrees, financial accounts, medical procedures, insurance claims, votes, transactions between smart objects, and anything else that can be expressed in code." 95

Many of the examples raised by Swan and the Tapscotts can be considered as the identifying markers that create a 'verifiable representation of who you are' as discussed in the preamble. What blockchain allows for, however, is the verifiability of the authenticity of these identifying markers without the need for any form of centralized control. Just as how criminals can trust that the money is where it is supposed to be on the bitcoin network, individuals could hypothetically trust that their identifying documents are legitimized, stored, and accessible on a DLT.

Several countries are either already using forms of blockchain technology or moving towards it; Estonia's digital identity program, for instance, claims that they have been experimenting with blockchain technology since before the Bitcoin white paper was even

\footnotetext{
${ }^{94}$ Melanie Swan, Blockchain: Blueprint for a New Economy (Sebastopol: O’Reilly Media, 2015). viii

95 Don Tapscott \& Alex Tapscott, Blockchain Revolution: How the Technology Behind Bitcoin is Changing Money, Business, and the World (New York: Penguin, 2016).
} 
released, referring to it as hash-linked time-stamping. ${ }^{96}$ Since 2012 though, they have been employing a unique form of DLT as a means of protection for data registry, including "the national health, judicial, legislative, security and commercial code systems, with plans to extend its use to other spheres such as personal medicine, cyber-security and data embassies."97

Crucially, unlike other social network entities, no sensitive data is actually stored on this particular version of a blockchain; the blockchain just stores 'hash values' which serve as 'digital fingerprints of the original data'. ${ }^{98}$ Thus, while the user can utilize the hash value to unlock their sensitive data, the hash value in itself does not hold any identifying features - just as a fingerprint can be identified to the person but contains no information about their gender, height, race, and so on. ${ }^{99}$ While Estonia does use forms of DLT to protect this data, it is also important in this context to differentiate Estonia's permissioned state-led blockchain from the core concept that will be presented at the oust of this section.

Estonia does not use a 'public and permissionless' blockchain for the management of its identity program. This type of blockchain (typified by Bitcoin) is a network in which any computer or system can become a node capable of viewing, writing, verifying, or authenticating a block. ${ }^{100}$ Each block further holds a copy of all of the transactions in the form of a ledger. Though it is the most popular and most tested version of a blockchain, it has inherent weaknesses in that the current consensus protocols - PoW in the case of Bitcoin, though others have

\footnotetext{
${ }^{96}$ European Union European Regional Development Fund, Frequently Asked Questions: Estonian Blockchain Technology.

97 Ibid., 1

98 Ibid., 2

99 Ibid.

100 Dave Bryson, Dave Penn, David C. Goldenberg \& Gloria Serrao, "Blockchain Technology for Government" in Mitre Technical Report (2018). 8
} 
developed far more efficient mechanisms - require an incredible amount of global computing power and energy with a relatively slow verification of transactions. ${ }^{101}$

Estonia, on the other hand, employs what it calls Keyless Signature Infrastructure (KSI) as a means of protecting its citizens' data. ${ }^{102} \mathrm{KSI}$ utilizes what can be referred to as a 'permissioned' blockchain, where consensus is based on "the idea that a preselected, authorized group of validators will create, verify, and attest to new blocks...the validators responsible for building the blockchain, as well as the participants in the network, are selected by the group and held accountable for their actions."103 The validators in the case of Estonia are essentially state agents, and the system's public key infrastructure (PKI) allows government officials to view or modify a citizens data. ${ }^{104}$ With that being said though, based on DLT and the public ledger (known as Guardtime), any viewing or changing is recorded on the immutable blockchain which is accessible to the citizen. ${ }^{105}$ Thus, the citizen has the ability to see who viewed their information and when, and they can challenge and prosecute that official if necessary. ${ }^{106}$

Important to know about PKI and KSI though is that Estonia does not utilize a centralized 'master database.' ${ }^{107}$ Rather, data is stored where it is created and it is not duplicated; if an organization or a state agency is to require a citizens data out of their own purview and they are authorized to do so, they can use an open source data exchange layer called 'The X-Road' to

\footnotetext{
${ }^{101}$ Nitesh Emmadi \& Harika Narumanchi., "Reinforcing Immutability of Permissioned Blockchains with Keyless Signatures' Infrastructure" in Proceedings of the 18th International Conference on Distributed Computing and Networking (46, 05 January 2017). 3

102 European Union European Regional Development Fund, Frequently Asked Questions.

103 Bryson, Blockchain Technology for Government, 5-8

104 Kasper Korjus, "Welcome to the Blockchain Nation" in Medium (7 July 2017).

${ }^{105}$ ABI Research, Blockchain-Based Cybersecurity (2017). 14

106 Korjus, Welcome to the Blockchain Nation.

107 lamsysteemiamet, "X-Road Introduction (Long version)" Youtube video (10 June 2016).
} 
securely access this data. ${ }^{108}$ The data that is being accessed, however, is stored and hosted locally by the server that created it in a single chain. While an authorized state agency can view the data, they cannot replicate the chain and thus their node is not hosting the entirety of the database. Each node hosts what they create - a hypothetical example would be that the medical office hosts medical history, and while an insurance agency can access a particular citizens data, the insurance node certainly does not host the entirety of Estonia's medical history. This is why the Nordic Institute for Interoperability Solutions has deemed that "There is no blockchain technology in the X-Road." ${ }^{109}$ Still, blockchain has become a sort of all-encompassing catchphrase for DLT, and broadly speaking, permissioned or public DLTs generally fall under the purview of blockchain. And further, while Estonia's program is state-led and permissioned, it is still highly-representative of alternative systems for identity management in a more decentralized manner.

With that said, other organizations are leveraging the usefulness of blockchain technology in their programs, such as the UN who have teamed up with the World Identity Network (WIN) to address the tragic issue of stateless and 'invisible' children with regards to human-trafficking. ${ }^{110}$ UN Women Deputy Executive Director and UN Assistant Secretary General Yannick Glemarec stated that "Leveraging blockchain technology offers potentially powerful solutions to address this serious challenge and save the lives of millions of children." 111 The UN thus issued a 'Global Challenge' in the form of open submissions to develop a program

\footnotetext{
108 Petteri Kivimaki, "There is no blockchain technology in the X-Road" in Nordic Institute for Interoperability Solutions (26 April 2018).

109 lbid.

${ }^{110}$ World Identity Network, "World Identity Network and United Nations team up to launch innovative blockchain pilot to help prevent child trafficking" in PRNewswire (10 November 2017).

${ }^{111}$ Neil Hughes, "UN will use blockchain and digital identities to combat child trafficking" in One World Identity (13 November 2017).
} 
utilizing blockchain technology that addresses the issue of child-trafficking in Moldova. ${ }^{112}$ In fact, the UN is already employing blockchain technology in its humanitarian efforts; since 2017, blockchain has been used to manage the identities of some hundred thousand Syrian refugees in Jordan with projections of managing all 500 thousand refugees in the country by the end of $2018 .^{113}$

The companies Microsoft and Accenture have also embraced blockchain technology in partnership under the ID2020 alliance of the UN, unveiling a prototype in June of 2017 at the UN headquarters of New York regarding how they could use blockchain technology for the 1.1 billion people without identity. ${ }^{114}$ The World Bank fostered organization ID4D and the Digital Impact Alliance (DIA) have entered the discussion as well with a 2018 document titled “Technology Landscape for Digital Identification” which features a comprehensive section on the uses of blockchain technology and DLTs. ${ }^{115}$

In this document, the DIA highlights the different forms of permissioned and public blockchains and presents the important notion of self-sovereign digital identity (SSDI). Being that there is a vast variety of different blockchains and a digital identity would theoretically have to navigate many of them (to be explained), the concept of an SSDI is an identity that belongs solely to the individual which is not dependent on a single authority outside of the individual. Further, it must also be something that cannot be arbitrarily or abruptly taken away. ${ }^{116}$ This seemingly corresponds with Point 5 of ID2020's manifesto that states “We believe that

\footnotetext{
112 Unite Ideas, Blockchain for Humanity Global Challenge (2018).

113 Russ Juskalian, "Inside the Jordan refugee camp that runs on blockchain" in MIT Technology Review (12 April 2018).

${ }^{114}$ Anna Irrera, "Accenture, Microsoft team up on blockchain-based digital ID network" in Reuters (19 June 2017 ).

${ }^{115}$ World Bank, Technology Landscape for Digital Identification.

116 lbid., 65
} 
individuals must have control over their own digital identities, including how personal data is collected, used, and shared. Everyone should be able to assert their identity across institutional and national borders, and across time. Privacy, portability, and persistence are necessary for digital identity to meaningfully empower and protect individuals." 117

To have control over one's identity is the key concept of SSDI, and the DIA references a GSMA blog to explain what this would look like. ${ }^{118}$ According to the blog, identity "is not a singular characteristic but rather a set of attributes that vary by relationship and moreover the plurality of these relationships can enhance the confidence level that the identity being asserted is genuine through corroboration."119 A government official can verify the passport of an individual which can be corroborated with the individuals bank account being that financial institutions are given a certain modicum of power to 'know your customer' (KYC). The two of these combined enhance 'the confidence level of the attributes associated with a given identity and hence the identity itself.' 120

To create the idealized vision of SSDI in the digital world on a blockchain - it is important to remember that this is hypothetical and idealized - a user would create a selfasserted block containing hashed identity attributes and a 'public key' to be signed by the user's private key. Other organizations refer to this self-asserted block as a container. ${ }^{121}$ The user would then be in complete control of the identity attributes that are hashed being that - as romanticized by the Estonian example - the data in itself is just a 'fingerprint' of the relevant

\footnotetext{
117 ID2020, The Alliance Manifesto (2019).

118 World Bank, Technology Landscape for Digital Identification, 66

119 Gautam Hazari, "The Relationship Between Blockchain and Digital Identity" GSMA (10 November 2016).

120 Ibid.

${ }^{121}$ Caribou Digital, Private-Sector Digital Identity in Emerging Markets (Farnham: Caribou Digital Publishing, 2016). 19
} 
data requiring a private key to unlock. The public key would then resemble a user id while the private key would be similar to a password (perhaps only accessible through biometrics). A relevant institution could then be given access to the personal data if the user gives them access with their private key and the institution could verify that the data is accurate. ${ }^{122}$

For instance, "the Passport Office could sign the hashed address, name, and photograph of the subject if the attribute values asserted by the user match those on record at the Passport Office."123 Most importantly though, while the passport office was given access and knows your name, address, and photograph, it certainly does not mean that anyone who sees your 'container' would have access to that information - you would have to use your private key to give them that information. What they would see, however, is that the Passport Office has signed your hash and thus has verified that you have an identity and are a 'real person'. ${ }^{124}$ This transactive verification would then be placed on the irrefutable and immutable blockchain.

Other institutions could also engage with these same sorts of transactive verifications. A financial institution could view your public key and see that you have been verified by the Passport Office which would allow you to open a bank account. This would then be stamped onto your public key, and an insurance broker, say, could see that you have both a real identity and bank account and would thus allow you to take out insurance - which would also be stamped onto your public key. These stamps would continue to accumulate and would further and further authenticate the user's block as a legitimate individual with a varying collection of credentials. The GSMA refers to this as 'reputation capital'. ${ }^{125}$

\footnotetext{
122 Hazari, The Relationship Between Blockchain and Digital Identity.

123 Ibid.

124 Ibid.

$125 \mathrm{Ibid}$.
} 
This collection of credentials would effectively accumulate into a verifiable representation of 'who you are'. What it allows for though - and what makes it so epochal - is that it empowers the user to own and control their block without the need of a centralized managing force. Though the identity would still be dependent on trusted institutions to provide the credentials, this particular idealized version of the blockchain would mean that the user would not be dependent on any one institution to provide their identity; rather, they can turn to a wide variety of mediums to develop their reputation capital. Further, the identity is not held and managed by a centralized force such as a government, and instead it is managed and verified by all of the institutions contributing to it and therefore none of them.

In the words of a Digital Caribou report titled 'Private-Sector Digital Identity in Emerging Markets:'

The individual creates an identity "container" that allows them to accept attributes or credentials from any number of organizations, including the state, in a networked ecosystem that is open to any organization to participate (e.g., to issue credentials). Each organization can decide whether to trust credentials in the container based on which organization verified or attested to them; in other words, a mortgage company may accept a credential issued by a leading global bank, but not one issued by a local bank. Importantly, this model does not require a state-based credential to be initiated (the state credential can be added at a later time, or not at all). ${ }^{126}$

In this hypothetical system, according to the World Bank, "the integrity of IDs based on a blockchain depend on the integrity of documents or data used to onboard an individual onto a blockchain (i.e., the verifiable claims)." ${ }^{127}$ Importantly though, the relevant institutions providing the documents will likely be using their own forms of blockchain to secure and hold these same documents - similar to the single chains hosted by Estonia's institutions (with the

\footnotetext{
${ }^{126}$ Caribou Digital, Private-Sector Digital Identity in Emerging Markets, 19.

127 World Bank, Technology Landscape for Digital Identification, 67.
} 
obvious difference that the X-road project is state initiated). Thus, the idealized vision of SSDI proposed by the GSMA blog (and therefore vicariously endorsed by the World Bank) would seemingly be a blockchain that records a user's navigation through a variety of other chains in a manner similar to the X-road; verifiably accumulating the credentials bestowed upon them by these trusted third parties. In sum, it would be this accumulation that is the user's self-owned digital identity, and it would be the user and the user alone who in control of it - as opposed to the Estonia example where it could be said that it is the Estonian state that is still managing the user's identity.

\section{The Realizations of Self-Sovereign Digital Identity}

The notion of an SSDI thus has to do with a user's self-navigation through various digital networks as means of building their reputation capital. If this were to be properly developed towards the idealized vision presented in the previous section, it could have very significant implications. In envisioning this, we can turn again to the Estonia example to help in the conceptualization - though it is again essential to differentiate the Estonian project from concepts of SSDI being that digital identity in Estonia is a state-led project employing a permissioned blockchain as a means of protecting citizens' data rather than self-sovereign ownership on the part of the citizens. Nevertheless, Estonia is still at the forefront of pioneering new conceptions of nationhood and governing as it interweaves itself with the digital realm, and what they have done illuminates some of the potentials of digital identity in an evolving world.

As of December 1, 2014, Estonia has developed what it is referring to as its E-residency program: 'The New Digital Nation.' ${ }^{128}$ What this program allows for is citizens of other

\footnotetext{
${ }^{128}$ Republic of Estonia. The New Digital Nation.
} 
nationalities to become a 'virtual resident' of Estonia, where they can gain access to a variety of E-services provided by the Estonian government via a state-issued digital identity represented by an electronic smart card (eID). ${ }^{129}$ Though Estonia has a population of 1.3 million, they have set the ambitious goal of adding 10 million e-residents to their 'digital nation' by $2025 .{ }^{130}$

According to the program director Kasper Korjus, since they have initiated the program, 50 thousand individuals from 165 countries and territories have applied and set up approximately 6 thousand new companies. ${ }^{131}$ E-residency has also been granted to notables such as German Chancellor Angela Merkel and the Prime Minister of Japan Zhinzo Abe. ${ }^{132}$

According to the program, an e-resident of Estonia "can establish and manage a locationindependent company online from anywhere in the world; establish a trusted EU company online in one day; manage the resulting company fully online; apply for a business bank account and conduct secure e-banking; access international payment service providers; digitally sign and transmit documents; and declare Estonian taxes online." 133 And though the e-resident is not an official citizen of Estonia, included benefits and motivations are membership of the EU, NATO, OECD, and the Eurozone, which gives the user access to new and stabilized markets as well as the ability to trade in euros. ${ }^{134}$

Though the program is evolving, users seeking an e-residency typically have to go to an Estonian embassy where they verify their national identity, provide a motivation statement, and

\footnotetext{
129 Piia Tampuu \& Anu Masso, “'Welcome to the Virtual State': Estonian e-residency and the digitalised state as a commodity" in European Journal of Cultural Studies (21/5, 2018). 544

130 Kaspar Korjus, "Estonian President Kersti Kaljulaid reveals the future direction of e-Residency" in Medium (18 December 2018).

131 Ibid.

132 Ibid.

133 E-Estonia, E-identity (available at https://e-estonia.com/solutions/e-identity/e-residency) 134 Ibid.
} 
pay a fee of 100 euros. While this shows an obvious current need for 'real world' institutions in order to verify the individual's identity, it also hints to a near-future hypothetical; if users had their own form of SSDI, they would theoretically be able to add 'Estonian E-Citizen' to help strengthen their reputation capital. Further, if other networks were to develop similar to that of Estonia's digital nation, a verified digital identity provided by Estonia - being that it is so undeniably secure and legitimate - could theoretically allow that individual onto these other networks in a trusted manner.

Early hints of the potentials of these networks can be found in the aforementioned regulation No. 910/2014 passed by the EU, which seeks to create a standardized underlying criterion that allows for the interoperability of one EU nation's digital identity to be used within another EU nation. More directly, however, on February 7th, 2018, Estonia's neighbor in Finland connected their data exchange layer with the X-road of Estonia to allow for expedited and secure data transfer between the two friendly countries. ${ }^{135}$ The head of the Project Management Department at Information System Authority, Tuuli Parenson, referred to it as "a radical step in a great many ways. It's a powerful sign of trust in technology, processes, people and the state as a whole, which cannot be taken for granted even in Europe."136 Other countries that are employing X-road are Azerbaijan, Namibia and Faroe Islands, though they have not directly linked their programs with Estonia. ${ }^{137}$

\footnotetext{
135 Finland Population Register Centre (Press release), Finland's and Estonia's data exchange layers connected to one another on 7 February - the rapid exchange of information between the countries is now possible (8 February 2018).

${ }^{136}$ E-Estonia, Estonian-Finnish e-Governance without borders (available at https://e-estonia.com/estonian-finnishe-governance-without-borders/).

${ }^{137}$ E-Estonia. Interoperability Services (available at https://e-estonia.com/solutions/interoperability-services/).
} 
The Estonian and the Finnish government refer to their program as 'e-governance without borders. ${ }^{, 138}$ Estonia is not just partnering with other countries, however - which is probably the more radical hint towards coming changes in the digital landscape. Indeed, in 2017 Estonia partnered with the organization Bitnation, which could perhaps be seen as a contention to Parneson's notion of trust in the 'state as a whole'. Indeed, Bitnation is a decentralized blockchain organization that refers to itself as a 'Decentralized Borderless Voluntary Nation' $(\mathrm{DBVN}) .{ }^{139}$ Estonia turned to Bitnation to provide a public blockchain notary for their eresidents, where citizens can publicly register birth certificates, marriage licenses, business contracts, and so on onto the blockchain. ${ }^{140}$

The vision of Bitnation is a "post-Nation State world of Voluntary Nations, City States and Autonomous Communities which compete for Citizens by providing a range of opt-in governance services." What Bitnation allows for is the ability for any individual to create a 'virtual nation' which can be joined on a voluntary basis by any other individual, regardless of geographical location, ethnicity, or any other category of populations - though a DVPN does not have to be based entirely in the virtual world. ${ }^{141}$ These nations can operate under the parameters of any set of rules designed by the creator or collection of creators, and one of the more important distinctions acknowledged by the organizations is that users have the ability to 'opt-in and opt-out'. ${ }^{142}$ The creation of a DVPN involves transactional rules maintained on a blockchain, creation of a constitution, choice of legal code (such as common law, civil law, Sharia), choice of economic model (capitalism, socialism, communism), choice of decision-

\footnotetext{
138 E-Estonia. Estonian-Finnish e-Governance without borders.

139 Bitnation, Bitnation Governance 2.0 (5 March 2018). 4

140 Luke Parker, "Bitnation starts offering blockchain public notary service to Estonian e-Residents" in Brave New Coin (30 November 2015).

${ }^{141}$ Bitnation, Bitnation Governance 2.0, 7

142 Ibid.
} 
making model (democracy, autocracy, theocracy), choice of jurisdiction, definition of governance services, and so on. ${ }^{143}$

Typically, these DBVNs operate under the decentralized jurisdiction of Pangea, which is the core infrastructure upon which voluntary nations can be "created, joined, and lived in. ${ }^{144}$ Pangea is the underlying technology that allows for citizens to "make peer-to-peer agreements, resolve disputes, and access governance services from DBVNs using the legal code of their preference. ${ }^{145}$ Within Pangea, every entity (citizen, organization, or even AI) is given a reputation score that can be searched by other platforms - not so dissimilar to the concept of reputation capital. ${ }^{146}$ And perhaps incredulously to some, On October 9, 2018, Bitnation announced that it would be releasing Vienna Convention compliant passports (ICAO and ISO/IEC 7810 ID-3 standard and machine readable) and has begun the process to get the passports recognized as 'Laisse-Passer' as seen with passports issued by national governments. ${ }^{147}$ The passports use the Bitnation Jurisdiction app's public key as a unique identifier. ${ }^{148}$

Though the process is certainly far from complete, the example of Bitnation represents an entirely new possibility in the realm of developing an officialized sort of identity. While Bitnation has a very disruptive vision centered on conceptualizing alternatives to the Westphalian state system, ${ }^{149}$ the fact that they are developing a reputation system to verify an

\footnotetext{
143 Ibid., 28

144 Ibid., 6

145 Ibid.

146 Ibid., 21-22

${ }^{147}$ Bitnation (Press Release), "Bitnation releases first batch of Vienna Convention compliant diplomatic blockchain passports" in Steemit (November 2018).

148 Ibid.

${ }^{149}$ Bitnation, Bitnation Governance 2.0, 10-14
} 
individual which allows that individual to collect credentials bestowed upon them from the digital nations on their network - all of which is searchable via other platforms - speaks to a very early and fascinating realization of SSDI. Moreover, Estonian e-residents can also use Bitnation's public blockchain to notarize their documents, highlighting a sort of cross-over between state-led projects and more autonomous ones.

Bitnation is not the only autonomous organization conceptualizing and developing forms of SSDI, however. Indeed, an organization called Evernym is creating a fully open source selfsovereign identity platform on a permissioned distributed ledger referred to as Sovrin. What Evernym and Sovrin are looking to do is to provide a trusted infrastructure that would allow a user to create a self-sovereign container from which an individual could collect verifiable claims in the transactive manner described in the previous section. ${ }^{150}$ Since its development, Evernym has partnered with The U.S. Department of Homeland Security, U.S. State healthcare programs, the UK National Health Services, as well as a number of financial institutions. ${ }^{151}$

What makes The Sovrin Identity Network somewhat unique in the decentralized blockchain world is that it would be dependent on a human governance layer to act as validators capable of verifying and 'permissioning' the transactions that would make up an individual's digital identity. ${ }^{152}$ While this permissioned form of DLT technically operates outside the realm of blockchain as it is traditionally understood, Evernym is entirely dedicated to the concept that "for identities to be truly self-sovereign, the nodes of the Sovrin Identity Network cannot be owned or controlled by any single company, organization, or government. Instead they must be

\footnotetext{
150 The Sovrin Foundation. Sovrin ${ }^{T M}$ : A Protocol and Token for Self-Sovereign Identity andDecentralized Trust (January 2018).

${ }^{151}$ ABI Research, Blockchain-based Cybersecurity (2017). 10

152 The Sovrin Foundation, The Inevitable Rise of Self-Sovereign Identity (29 September 2016). 13
} 
distributed around the world, operated by trusted institutions in multiple countries and industries, forming a decentralised network much like the Internet itself [sic]." ${ }^{\prime 53}$ The organization has developed the not-for-profit organization 'The Sovrin Foundation' which is dedicated exclusively to governing the system, and they have further claimed that "Over time, the Sovrin Foundation anticipates that stewards will include financial institutions, universities, healthcare providers, telcos, NGOs, governments, and other institutions in a position of public trust who wish to support a self-sovereign identity layer for the Internet." 154

Another seminal organization working towards digital identity is that of uPort, which operates under the Consensys umbrella on the Ethereum blockchain. While Ethereum (and Consensys) will be discussed more in depth in the following chapter, the creators of uPort have argued that decentralized identity is vital to the scaling of this particularly important blockchain. ${ }^{155}$ uPort is a highly-developed fully open-source organization sharing a similar philosophy regarding the need for SSDI to that of Evernym, but differentiate themselves in that they are building their technology on the public and permissionless blockchain of Ethereum. ${ }^{156}$ Users in this system will be able to self-generate a block that is universally discoverable whilst storing encrypted data regarding their private and personal information locally on their smartphone (or perhaps in a cloud). ${ }^{157}$ In 2016, they partnered with Microsoft under the ID2020 mandate looking to develop a 'cross-chain identity solution that can be extended to any future blockchains or new kinds of decentralized, distributed systems. ${ }^{158}$ Microsoft further stated

\footnotetext{
153 Ibid.

154 The Sovrin Foundation, The Technical Foundations of Sovrin (29 September 2016). 6

155 Kames, "Why Decentralized Identity Is Essential To Scaling the Ethereum Blockchain" in Medium (20 June 2018 ).

${ }^{156}$ Christian Lundkvist, Rouven Heck, Joel Torstensson, Zac Mitton \& Michael Sena, uPort: A Platform for SelfSovereign Identity (20 October 2016).

${ }^{157}$ Kames, "The Basics of Decentralized Identity" in Medium (26 June 2018).

158 Yorke Rhodes III, "What does identity mean in today's physical and digital world" in Microsoft Azure (31 May 2016).
} 
"[We] can imagine a world where an individual can register their identity in a cross blockchain fashion, providing a single namespace for lookup regardless of blockchain of choice...[We are] excited by the potential societal benefits that can be derived from an identity that transcends borders, blockchains, organizations and companies."159

Further, in November of 2017, uPort announced that they would be partnering with the city of Zug, Switzerland to pilot the first experimentation with government-issued SSDI. ${ }^{160}$ The way it works is that citizens of Zug download the uPort mobile app and then create an account. Upon this creation, the uPort app gives the user a private key and then creates two blocks on the Ethereum network that serve as an identity hub. The user would then log into the Zug ID web portal by scanning a QR code, and following this would have to meet with a city clerk at the city's registration office for an in-person verification utilizing a national identity card such as a passport. Upon this completion, the citizen is officially on the Zug network. ${ }^{161}$ In June of 2018 , Zug held the first municipal election taking place on a blockchain. ${ }^{162}$ At the outset of this experiment with digital identity, uPort announced "Our partnership with Zug is one giant step towards our goal of realizing a global self-sovereign identity system."163

Another organization at the forefront of SSDI on the blockchain is Digital Bazaar who have fostered the Veres One Blockchain to serve as a "a fit-for-purpose blockchain optimized for identity on the Web." ${ }^{164}$ Veres One is similar in vision to both Evernym and uPort with a primary focus on what they refer to as decentralized identifiers, but they differ from the others in

\footnotetext{
159 Ibid.

160 Paul Kohlhaas, "Zug ID: Exploring the First Publicly Verified Blockchain Identity" in Medium (6 December 2017).

161 uPort, "First official registration of a Zug citizen on Ethereum" in Medium (15 November 2017); Kohlhaas, Paul, Zug ID: Exploring the First Publicly Verified Blockchain Identity.

162 Alexandra Wey, "Switzerland's first municipal blockchain vote hailed a success" in Swiss Info (2 July 2018).

163 uPort, Zug ID.

164 Veres One, The Veres One Project (2018).
} 
that they are a freestanding public and permissionless blockchain (as opposed to uPort which is based on Ethereum and Evernym which is permissioned). The founding organization is also under contract with the United States Department of Homeland Security. ${ }^{165}$

Civic is another group that should be considered as part of the discussion, who provides a security identity platform (SIP) that allows for multifactor authentication on websites and mobile apps. ${ }^{166}$ This could be seen as similar to SSO, with the difference being that new accounts are created on a DLT by the true owner of the identity data, which is then used to log in to other sites and apps - as opposed to using a centrally owned Facebook account to log on to other sites. ${ }^{167}$ The organization Blockauth operates under a similar parameter being that it creating a network of OpenID/OAuth login providers to verify a user's claims, allowing institutions to outsource their KYC requirements to BlockAuth. ${ }^{168}$ Other incredibly interesting organizations in the field of SSDI are Democracy Earth and Aragon, but they will be discussed more in depth in the following chapter and conclusion.

Beyond these organizations, there are perhaps hundreds of different blockchains that are seeking to address and provide solutions to the issues regarding digital identity - many of which do not have fully fledged out concepts so much as they have grandiose ideas. Further, many organizations are predominantly concerned with the expediting of KYC services and trusted exchange rather than the provision of the idealized SSDI as we have discussed. Nevertheless, the examples of Bitnation, Everynym, uPort, and Digital Bazaar show that the notion of SSDI

\footnotetext{
165 Ibid.

${ }^{166}$ Civic, Secure Identity Platform (2019).

167 Ibid.

168 Atif Ghulam Nabi, “Comparative Study on Identity Management Methods Using Blockchain” in University of Zurich (29, September 2017). 12
} 
facilitated through the blockchain is now not so much a hypothetical as it is something seemingly on the horizon; though, it should be noted that it is still very much in its infancy.

With all of this being said, the overarching concept of SSDI again has to do with a user's navigation through a variety of networks and the accumulation of 'reputation capital' as they interact with these networks. Most importantly though, as argued by the Digital Caribou report, the creation of identity containers "Does not require state-based credentials to function-the state is just one of many different entities that can attest to a credential that is placed within the individual's identity container." 169 The organization actually likens it to the Aadhar program of India; even though the Aadhar number is obviously state-backed, all that is issued is a unique number which does not bestow legal status, but simply allows each government agency to issue official credentials on top of it. ${ }^{170}$ Digital Caribou makes the case that Everynym is effectively mirroring this concept with the key exception that they are not state-backed. ${ }^{171}$

Recognizing that the SSDI would theoretically allow individuals to create a legal identity without the backing of any particular state has severe implications for the very notion of citizenship - especially when combined with the aforementioned notion presented by ID2020 that says 'everyone should be able to assert their identity across institutional and national borders'. Thus, these developments inspire discussions regarding the very nature of citizenship in an evolving world that is ever more intersecting with the digital. Interestingly though, these discussions did not necessarily emerge with the release of Bitcoin in 2008 and the development of blockchain technology. Rather, they came with the development of the internet itself, where those interested in cryptography have long been proclaiming that digital networks would spell

\footnotetext{
${ }^{169}$ Caribour Digital, Private Sector Digital Identity in Emerging Markets, 97

170 Ibid.

${ }^{171}$ Ibid.
} 
the end of nations as sovereignty is passed from the state to the digital individual. Enter the cypherpunks.

\section{Digital Nations and Virtual Worlds}

"The assumption that what currently exists must necessarily exist is the acid that corrodes all visionary thinking."

- Murray Bookchin

Although the notion of SSDI (also commonly referred to as self-sovereign identity) is mostly associated with DLTs, a very strong relationship could and should indeed be identified between the organizations and proselytizers of today and the dreamers of old; those prominent figures in the earlier days of the internet who sought to develop strong cryptography to protect individual identities on the internet. These prominent figures fought to in what was referred to as the 'crypto wars' of the late 1980s and 90s, competing against governments who were trying to suppress the technology and keep it within their own purview. Rather than allowing this, these individuals recognized the power associated with anonymity and pseudonyms on the internet, fighting back because they believed that strong cryptography and cyphers would serve as a liberating force for individuals - both protecting them from corrupt officials and allowing them self-determination to collaborate in voluntary association over the world wide web. ${ }^{172}$

These individuals referred to themselves as the 'cypherpunks' and they fought under a political philosophy called crypto-anarchism, where the term 'anarchy' represented freedom from coercion by external forces in the form of central control. ${ }^{173}$ In the minds of the cypherpunks, cyberspace was symbolic of a potential alternative structure of collaboration where

\footnotetext{
172 Ibid., 66-70

173 Timothy May, "Crypto Anarchy and Virtual Communities" in Crypto-Anarchy, Cyberstates, and Pirate Utopias, ed. Peter Ludlow (Cambridge: MIT Press, 2001), 69
} 
there was 'no rulers, no leaders, and no laws.' ${ }^{174}$ Thus, they hid under the guise of pseudonyms and developed networks and systems that protected privacy from governments, corporations, and other 'large, faceless organizations' who would not provide these freedoms based on their own beneficence. ${ }^{175}$ The most critical concept in these wars was indeed privacy, and the cypherpunks and crypto-anarchists considered it to be near-synonymous with freedom. ${ }^{176}$ In 1993 , one of the more influential thinkers in the movement, Eric Hughes, described it in The Cypherpunk's Manifesto as "the power to selectively reveal oneself to the world." 177 In accordance with this, the seminal thinker who coined the term crypto-anarchism, Timothy May, questioned why anyone should have to reveal their 'true name' in cyberspace. ${ }^{178}$

The idea of a 'true name' is a concept borrowed from the highly influential technologist and science fiction writer Vernon Vinge. In the story True Names, released in 1983, Vinge portrayed a digital world of virtual reality, where real world hackers existed as warlocks on an 'other plane' (digital realm) of magic with pseudonyms such as Mr. Slippery and The Mailman. In this fictitious world, the most important thing for a warlock was to protect their real-world identity - their true name - for if it was discovered by the American Government or perhaps another warlock, that government or warlock would be able to coerce the individual in the real world under the threat of violence.

In lieu of this sort of thinking, governments were seen simply as a coercive and violent force; this is no more apparent than in John Perry Barlow's 1996 seminal and infamous

\footnotetext{
174 Ibid.

175 Eric Hughes, “A Cypherpunk's Manifesto" in Crypto-Anarchy, Cyberstates, and Pirate Utopias, ed. Peter Ludlow (Cambridge: MIT Press, 2001), 82

176 Ibid.

177 Ibid., 81

178 May, Crypto Anarchy and Virtual Communities, 70-71
} 
document titled A Declaration of the Independence of Cyberspace, where he referred to cyberspace as 'the new home of the mind'. ${ }^{179}$ In this, he claimed that 'governments of the industrial worlds' had no authority over those who participated in the realm of cyberspace, and that the digital global social spaces are naturally independent with no ruler, no moral right for any individual to rule, and that liberty came from the new social contract that was being developed. ${ }^{180}$ He continued to say "Cyberspace consists of transactions, relationships, and thought itself, arrayed like a standing wave in the web of our communications. Ours is a world that is both everywhere and nowhere, but it is not where bodies live...we are creating a world where anyone, anywhere may express his or her beliefs...your legal concepts of property, expression, identity, movement, and context do not apply to us." ${ }^{181}$ He concludes by saying "Our identities have no bodies, so, unlike you, we cannot obtain order by physical coercion...We will create a civilization of the Mind in Cyberspace. May it be more humane and fair than the world your governments have made before."182

Timothy May also chastised the role of the government, questioning "Why do we so often accept the notion that governments issue us our names and our identities, and that government must ensure that names are true names?"183 Though his musing long predated blockchain, May might have found some solace in the notion of a self-created identity block that 'does not require state-based credentials to function.' Further, Hughes would likely approve of a self-owned identity container that allows one to selectively reveal their identity however and to

\footnotetext{
179 Peter Ludlow, Crypto-Anarchy, Cyberstates, and Pirate Utopias (Cambridge: MIT Press, 2001), 1

180 John Perry Barlow, "A Declaration of the Independence of Cyberspace" in Crypto-Anarchy, Cyberstates, and Pirate Utopias, ed. Peter Ludlow (Cambridge: MIT Press, 2001), 27

181 Ibid., 28

182 Ibid.

183 Timothy May. Cited from Janet Abate, "True Risks? The Pleasures and Perils of Cyberspace" in Science Fiction and Computing: Essays on Interlinked Domains, ed. David Ferro \& Eric Swedin (Mcfarland, 2011), 197
} 
whomever they want. Finally, they would both likely fight for the previously discussed developments regarding SSDI being that May stated in the 1988 Crypto Anarchist Manifesto that "The whole area of digitally-authenticated reputations, and the 'reputation capital' that accumulates or is affected by the opinions of others, is an area that combines economics, game theory, psychology, and expectations. A lot more study is needed."184

The study seems to be taking place, and it would also seem that crypto-anarchists and cypherpunks figuratively passed the torch onto some of the more radical and enthusiastic blockchain developers. Bitnation's white paper, for instance, references Timothy May when he said in 1994 that "A phase change is coming. Virtual communities are in their ascendancy, displacing conventional notions of nationhood. Geographic proximity is no longer as important as it once was." 185 Further, Susan Templehof, the founder of Bitnation, echoes similar sentiments to the original dreamers of digital communities and virtual nations; when John Perry Barlow stated in 1994 that "Governments derive their just powers from the consent of the governed. You have neither solicited nor received ours. We did not invite you,"186 Susan Templehof expanded by saying in 2015 that "Basically what we are doing is making nation state governments entirely irrelevant. And no government, whether democratic or autocratic, can survive without the consent of its subject."187

The anti-government animus has indeed long-existed in the world of cryptography, and it more often presents itself as forms of libertarianism and anarcho-capitalism; May himself states that the 'anarchism' of crypto-anarchism "is the same sense of anarchy used in anarcho-

\footnotetext{
184 May, Crypto Anarchy and Virtual Communties, 71

185 Bitnation, Bitnation Governance 2.0, 17

${ }_{186}$ Barlow, A Declaration of the Independence of Cyberspace, 27

187 Bitnation. Cited from Marcella Atzori, Blockchain technology and Decentralized Governance: Is the State Still Necessary? Published 2015. https://papers.ssrn.com/sol3/papers.cfm?abstract_id=2709713, 10
} 
capitalism, the libertarian free-market ideology that promotes voluntary, uncoerced economic transactions." 188 Further, he contends that "Crypto anarchy is the cyberspatial realization of anarcho-capitalism, transcending national boundaries and freeing individuals to make the economic arrangements they wish to make consensually." ${ }^{189}$ And finally, though they did not necessarily endorse it, even the creator of Bitcoin referenced their technology as being useful to conceptions of libertarianism. ${ }^{190}$

The appeal to libertarianism on the part of crypto-enthusiasts has to do with a fetishization of freedom for the individual and the elimination of external coercion. ${ }^{191}$ May cites Friedrich Hayek and David Friedman as the main inspirations for this particular political philosophy, where the former equates freedom with a system where "men could at least attempt to shape their own life, where man gained the opportunity of knowing and choosing between different forms of life [sic]." ${ }^{\text {192 }}$ The latter's highly influential father, Milton Friedman, revered Hayek's work and called it essential reading and a true classic. ${ }^{193}$ Milton Friedman also wrote one of the most influential books in American-style economics titled Capitalism and Freedom, where he makes the case that capitalism "emphasized freedom as the ultimate goal and the individual as the ultimate entity in the society." ${ }^{194}$ In Milton's mind, capitalists take “freedom of the individual... as our ultimate goal in judging

\footnotetext{
188 May, Crypto-Anarchy and Virtual Communities, 69

189 Timothy May. Cited from Janet Abate, "True Risks? The Pleasures and Perils of Cyberspace" in Science Fiction and Computing: Essays on Interlinked Domains, ed. David Ferro \& Eric Swedin (Mcfarland, 2011), 197

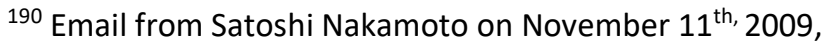
http://satoshi.nakamotoinstitute.org/emails/cryptography/12

191 May, Crypto Anarchy and Virtual Communties, 69

192 Friedrich Hayek, The Road to Serfdom (New York: Routledge Publishing, 1944), 15

193 Ibid., Introduction

194 Milton Friedman, Capitalism and Freedom (Chicago: The University of Chicago Press, 1962), 5
} 
arrangements. ${ }^{\prime 195}$ Further, they view the free market and private property as the only institutions capable of protecting human dignity and freedom. ${ }^{196}$

Whether or not there is merit to this particular market-based political philosophy is a matter I will take up in the following chapter. However, it is worth noting that the concept of an unfettered market facilitating free individuals has become fairly preeminent in the crypto world which has definitely trickled into the conceptualizing of SSDI. Bitnation, for instance, has declared that its vision is a "Global free market for governance services. A post-Nation State world of Voluntary Nations, City States and Autonomous Communities which compete for Citizens by providing a range of opt-in governance services. In our world sovereignty shifts decisively from the State to the Citizen, and humanity reclaims freedom of choice when accessing governance services." 197 They continue to say that "Free market arbitration paves the way for humanity to reclaim personal sovereignty."

This vision of a world where virtual nations vie for the citizenship of an SSDI through market-like mechanisms via voluntary opting in and out does indeed directly challenge historical notions of the role of the state in the provision of identities. Though the example of Bitnation is merely one realization of SSDI, as a concept it is still representative of an ambitious challenge to the Westphalian state system in that the notion that an individual should be able to choose what constitutes their citizenship flies directly in the face of a liberal world order premised on simply not having that choice.

\footnotetext{
195 Ibid., 12

${ }^{196}$ David Harvey, A Brief History of Neoliberalism (Oxford: Oxford University Press, 2005). 20

197 Bitnation, Bitnation Governance 2.0, 4

198 Ibid., 14
} 
In this, what we are referring to is the social contract - one of the most important and developed concepts of Western liberal political philosophy and pretty well the strongest justification for governments and states. ${ }^{199}$ The social contract essentially posits that individuals have consented, either implicitly or tacitly, to surrender some of their freedoms and submit to the authority of the ruler in exchange for the protection of their rights. The democratic blockchain theorist Rainer Baubock is correct to note, however, that "The social contract metaphor that has informed liberal thinking about citizenship since Hobbes, Locke and Rousseau is misleading in this respect. Citizenship as a legal status of membership in a territorial polity has never been based on consent. Citizenship in today's states is generally acquired at birth - either through birth in the territory or descent from citizen parents."200

Being that in a liberal-state-system individuals do not have any choice in regards to what social contract they abide, the challenge that SSDI poses is far more in line with the anarchism of anarcho-capitalism - where the original (some might say true) anarchists have long challenged the notion of a social contract. Indeed, the idea that people must sacrifice their freedom to govern themselves so that a society governed by others can exist is obviously quite far removed from the true liberty the anarchist knows to be the emancipation of humanity: "If the consent of every individual be necessary, in what manner is that consent to be given? Is it to be tacit, or declared in express terms?"201 These were the sorts of questions asked by the individual widely credited as the original visionary of political anarchism, William Godwin. ${ }^{202}$ Godwin recognized that the concept of giving one's consent was baseless being that the child is born into a society

\footnotetext{
${ }^{199}$ Wessel Reijers, Fiachra O’Brolchain, Paul Haynes, “Governance in Blockchain Technologies \& Social Contract Theories,"in Ledger.org (Vol 1, 2016). 138

${ }^{200}$ Rainer Baubock, "Citizenship in Cloud Cuckoo Land?" in Debating Transformations of National Citizenship, ed. Rainer Baubock (Switzerland: Springer Open, 2018). 264

${ }^{201}$ William Godwin, An Enquiry Concerning Political Justice (London: G.G.J. and J. Robinson, 1793), 143

202 Ibid.
} 
that has predetermined rules that relegate the individual as subservient to that in which they had no say and that in which they cannot opt out of; thus, being that there is no actual consent given, the laws of the society serve as the coercive ruler that restricts and denies an individual's right to self-determination. ${ }^{203}$ Being that anarchism is to be without a ruler, the laws of the social contract that coerce without consent are thus antithetical to the essence of true liberty. How can a contract be valid if it has not been consented to? This is the anarchist critique at its most rudimentary level.

Still though, in the words of Liav Orgad, "Under the current structure of international law, individuals exist as legal persons only through a status conferred to them by a state. Individuals are citizens or residents of some state; an international legal status of a 'human being' is non-existent." 204 What the current developments of SSDI are starting to render, however - which has seemingly been endorsed by the UN, World Bank, Microsoft, Accenture, Bitnation, Everynym, uPort, Digital Bazaar, and even somewhat indirectly through Estonian egovernance - is a notion of an intranational legal declaration of a person that is independent of any state validation. ${ }^{205}$ Further, the digital residency of Estonia and the development of DBVNs on the part of Bitnation (especially if they are able to develop the Pangea passport as planned) suggest an early conceptualization of statehood that could possibly exist in cyberspace. ${ }^{206}$ Finally, what both Bitnation and Estonia further illustrate is a desire (and perhaps capability) to move beyond the inflexibility of the Westphalian social contract by allowing SSDIs to opt-in and out of their virtual 'nations'.

\footnotetext{
203 Ibid., 143-146

204 Liav Orgad, "Cloud Communities: The Dawn of Global Citizenship?" in Debating Transformations of National Citizenship, ed. Rainer Baubock (Switzerland: Springer Open, 2018). 253

205 Ibid., 256

206 Ibid., 257
} 
With all of this being said, though - and in relation to the desires of the cypherpunks and the claims of organizations such as Bitnation - a vital aspect that needs to be considered in the discussion of SSDIs is that the concept of a global identification (legal or not) that allows somebody to 'opt-in and out of citizenships' is very different from an actual citizenship - as British Prime Minister Theresa May declared in 2016, "If you believe you are a citizen of the world, you are a citizen of nowhere. You don’t understand what citizenship means."207 Though this should certainly not be read as an endorsement of the Prime Minister's politics, her shrewd analysis is not incorrect; to conflate a notion of an international legal identity with the rights bestowed upon an individual based on their citizenship is simply misguided. Indeed, an oft-cited quote regarding the nature of citizenship is Hannah Arendt's 'the right to have rights.' In this, Arendt lamented any idealistic proclamation of universal and inalienable human rights (which I correlate to an international legal identity), knowing first hand that "the moment human beings lacked their own government and had to fall back upon their [inalienable human rights], no authority was left to protect them and no institution was willing to guarantee them." ${ }^{208}$ As Michael Blake bluntly puts it, the right to have rights "demands the existence of an agency that will deploy force against those who would presume to deny or trespass on those rights." ${ }^{209}$ This would be the protection aspect of the social contract.

In this, it does not really matter if one is abiding to Hobbes, Locke, or Rousseau; all three of the primary social contract theorists recognize that the state exists as a sovereign to protect the perceived fundamental rights of its citizens within the liberal world order - to protect and

\footnotetext{
207 Max Bearak, "Theresa May criticized the term 'citizen of the world.' But half the world identifies that way" in The Washington Post (5 October 2016).

${ }^{208}$ Hannah Arendt, The Origins of Totalitarianism (Berlin: Schocken Books, 1951), 292.

${ }^{209}$ Michael Blake, "Virtual Politics, Real Guns: On Cloud Community, Violence, and Human Rights" in Debating Transformations of National Citizenship, ed. Rainer Baubock (Switzerland: Springer Open, 2018). 291
} 
preserve property and lives of the citizens in the mind of Hobbes; to uphold and protect the natural rights of man in the mind of Locke; and to assure and guarantee rights, liberties, freedom, and equality in the mind of Rousseau. In order to do this, though, the state holds what is famously referred to as the 'monopoly on violence'; in the words of Max Weber, this means that the state claims "the monopoly on the legitimated use of physical force." ${ }^{210}$ This concept essentially refers to the militarized institutions of the police and the military who uphold whatever laws are determined by the state in which they are developed; this is why Hobbes refers to the state as the 'mightiest force' - the strongest entity guarantees the individual the right to have rights and that no individual can trespass on another's rights.

To make the claim, then, that developing digital networks will shift the sovereignty from 'the state to the citizen' requires a dependence on an individual's existence on the network that is either in no need of protection from a 'mightiest force' or predominantly exists as the mightiest force. While there is certainly remarkable potential in the technology (more of which will be discussed in the following chapter), Marcella Atzori makes the prescient argument that "in this phase individuals are not citizens yet: in spite of the sophisticated technologies they may use to create contractual arrangements, they are still living in a state of nature, in which the law of might - or the laws of the market - prevails on common good."211 Atzori's continues to argue that regardless of whatever mechanism is used to achieve collective consensus within a virtual nation, frictions and conflicts will inevitably arise. ${ }^{212}$ While many have looked to the technological developments in blockchain to resolve these sorts of conflicts, developing

\footnotetext{
${ }^{210}$ Max Weber, Weber's Rationalism and Modern Society, trans \& ed by Tony Waters and Dagmar Waters. (London: Palgrave Books, 2015).

${ }^{211}$ Marcella Atzori, Blockchain technology and Decentralized Governance: Is the State Still Necessary? (2015), available at https://papers.ssrn.com/sol3/papers.cfm?abstract_id=2709713. 23 212 Ibid.
} 
conceptions of digital nations (as desired by those in line with the cypherpunks) that seek to shift the sovereignty from the state to the citizen would still hypothetically be dependent on a sovereign force capable of protecting individuals against the trespass of others on their inalienable human rights and individual liberty - which is pretty well the exact justification of the state under social contract theory and thus leads to some form of coercive force utilizing the threat of violence.

While Atzori wrote one of the most robust and illustrious critiques of this sort of ideology, she chooses to open her article with a quote from Lawrence Lessig that - in this author's mind - poetically suggests the root of her concerns: "To push the antigovernment button is not to teleport us to Eden. When the interests of government are gone, other interests take their place. Do we know what those interests are? And are we so certain they are anything better?"213 The vision of Bitnation and the cypherpunks posits that it would be free market arbitration that paves the way for individuals to reclaim their sovereignty, which seemingly falls in line with anarcho-capitalist/libertarian beliefs (stemming from thinkers such as Hayek and Friedman) that capitulate private property and the free market as the only institutions capable of protecting individual human dignity and freedom.

What should be recognized here, however, is that the institution of private property is by far the most vital justification for the social contract. Again, it does not matter if one is abiding to Hobbes, Locke, or Rousseau, they all recognize the justification for the sovereign as being related to conceptions of human freedom being tied to the right to private property. Though the three differ in their conceptualization of the true nature of private property, they share in the

213 Ibid. 
belief that its protection is the primary purpose of government. What many in the blockchain world seem to be implying, however, is that strong cryptography facilitated by DLT that enable SSDI can serve as a means of 'enforcement' in the digital realm. Brett Scott provides a critique to this line of thinking, however, presenting the concept of a 'Techno-Leviathan' where automated code comes to replace the militarized institutions of the state; though this should be recognized as incredibly concerning being that technocrats reflecting the interests of the elites would be able to effectively game the system towards their own interests. ${ }^{214}$ In line with this and as put by Atzori, "In virtue of their technical skills, code developers, miners, fintech professionals and technopreneurs would easily have a privileged position in society, becoming the new policy makers to detriment of a big mass of computer illiterate or low skilled individuals, reduced to mere passive recipients of services." ${ }^{215}$ Atzori further highlights how even the Bitcoin network has effectively become centralized, where decisions are made and executed by a team of core developers and everything to do with governance of the network has to pass through the bottleneck of this elite, privileged class. ${ }^{216}$ Could this centralization not be replicated in what is being conceptualized as a digital nation?

Further, in a digital realm where the free market is to be the force in charge of providing the necessary protection for an individual's private property - regardless of whether it is done via algorithm or code - could it be said with certainty that this force would be preferable to that of some sort of state apparatus? Anarcho-capitalists famously call for the privatization of security institutions: in a system of digital networks vying for citizens to opt-in to their particular

\footnotetext{
${ }^{214}$ Brett Scott, "Visions of a Techno-Leviathan: The Politics of the Bitcoin Blockchain" in Eth Zurich Center for Security Studies (11 June 2014). 4

${ }^{215}$ Atzori, Blockchain technology and Decentralized Governance, 27

${ }^{216}$ Atzori, Blockchain technology and Decentralized Governance, 28
} 
jurisprudence, would the security this jurisprudence provides an SSDI be a determining factor in an individual's choice? Regarding SSDI, it is hard to deny that malicious attacks would be possible via hacking, for instance - though the technology is fairly remarkable in its resistance to this. Nevertheless, the possibility is there. Would everybody be able to afford the most sophisticated technologies to deny this trespassing against their fundamental right to selfsovereignty? If not, would those unable to afford it be protected? Are they really self-sovereign in that case? Further, are the ones who are paying for a private entity to act as the mightiest force actually sovereign?

Many in the cypherpunk fold also look to blockchain's capability to create self-enforcing contracts as a means of providing stability and protection (which will be discussed in far more detail in the following chapter). Per Scott, "Conservative libertarians hold tight to the belief that, if only hard property rights and clear contracting rules are put in place, optimal systems spontaneously emerge."217 In his critique of this thinking, however, he continues to say that "For much of modern history, the key issue with powerful institutions has not been their willingness to break contracts. It has been their willingness to use seemingly unbreakable contracts to exert power." In this, Scott worries that not only will elites be able to game the system, but they will be able to create systems of exploitation that once opted-into (perhaps by the code-illiterate) are incredibly difficult to break out of; or perhaps individuals will have to opt-in to not so optimal systems as a means of providing themselves basic protection.

It is for these sorts of reasons and more that a select group of blockchain enthusiasts are exercising sincere trepidation about some of the more grandiose claims in the blockchain world,

${ }^{217}$ Scott, Visions of a Techno-Leviathan, 4 
sharing Lessig's concern about the other interests that might takeover should we hit the 'antigovernment button'. All of this is not to say, however, that we should deny SSDI as a significant challenge to traditional conceptions of citizenship in an evolving world. Rather, it is my assertion - based on the above critique - that it is vital to ground the conceptualizations within the much safer challenge that SSDI poses rather than to irreversibly pledge ourselves to a dependence on unknown interests - especially ones facilitated by a free market. Regarding the safer challenge, then, Orgad still retains a significant level of optimism, for instance, in the notion of a global identity, realizing very clearly that "a global ID is not a status of citizenship nor does it create, in and of itself, an international legal status, although it is a prerequisite for it." ${ }^{118}$ Rather than this, Orgad argues an inspiring case that "an international legal persona should not be seen as a replacement of national citizenships but rather a complimentary to it."219

Returning to SSDI and the concept of an identity container to illustrate the above notion; the container in itself would not represent any form of citizenship that bestowed upon a person any form of legal rights culminating in the protection aspect of the social contract. Rather, it would serve as an acknowledgement that the person who holds the container 'belongs to humanity' - to borrow alternative phraseology from Arendt. ${ }^{220}$ While some might liken this to a form of citizenship (or perhaps even global citizenship), in reality it would more-so serve as a vessel upon which an individual could navigate actual citizenships and whatever we choose to identify these developing 'digital citizenships' as; the vessel does not guarantee the passenger anything except the ability to choose their own passage.

\footnotetext{
218 Orgad, Cloud Communities: The Dawn of Global Citizenship, 256

219 Ibid., 258

${ }^{220}$ A theme throughout Hannah Arendt, The Human Condition (Chicago: University of Chicago Press, 1958).
} 
In the developing cyberspace, this vessel could verify a more traditional citizenship, such as having the passport office stamp Canadian nationality onto your identity block or to be in possession of an Adhaar number; it could be something a bit more advanced, such as being an officialized resident of e-Estonia; or, they could be something different altogether, such as being a 'citizen' of a DBVN under the Pangea jurisdiction of Bitnation. In this, the SSDI would allow you to navigate all of these and you could be any of them, all of them, or - most importantly none of them. In the contemporary political and economic climate, this in itself should be considered epochal: where a user would hypothetically be able to choose the parameters of how they wish to present themselves on digital networks rather than being relegated to the identity of 'citizen of a particular country'. On a network where participation is not dependent on being a Canadian citizen, for instance, I could choose to present myself as a citizen of Pangea, or I could just present my identity container that anyone can see as legitimate. A user could thus verifiably control their predominant affiliations within cyberspace, which would indeed designate them as sovereign in the sense that they are free to choose who or what they want to be.

It should go without saying at this point, however, that this is not making the bold claim that SSDI facilitated by blockchain will come to replace 'The Sovereign' as it is traditionally understood under social contract theory in the Westphalian state system; further, at this point it is not clear that this would even be desirable. Rather than this, the incredible challenge that SSDI poses is simply that it allows for individuals to develop an irrefutable avatar that facilitates autonomous and verifiable participation on digital networks within cyberspace. While the nature of these networks will be covered in far more detail in the following chapter, it should be noted at this point that the networks calling for participation will not be limited within the parameters of what we have come to understand as governance, citizenship, and nationhood. Indeed, we 
could be talking about an entirely new understanding of socio-political relationships. And while there is surely a place for organizations that provide the more traditional governing-services (such as that of Bitnation), there is also the opportunity for entirely new 'communities of affinity' to emerge - which I would posit is the true awe-inspiring potential of blockchain. ${ }^{221}$ I would also say that it is not out of line to suggest that these developments could indeed be the key to transcending the social contract and to thus chip away at the power of the nation-state - a notion that I will be examining in the concluding chapter of this thesis. However, I would ultimately implore that we differentiate the developing virtual communities from that of a nation.

As the theory section of the following chapter will continue to discuss, the predominant justifications for the true nation-state has to do with the protection aspect of the social contract, which is propagated by a set of underlying assumptions that - I will ultimately argue - should not be transposed onto the new world that blockchain can create; of these assumptions, it is that of 'the right to private property' seen as being synonymous with an individual's freedom that blockchain has the potential to transcend - the same right that is most preeminent in the justification for the construction of a social contract in a nation-state. Thus, if the following section is able to successfully convince the reader that decentralized networks managed by a blockchain serves as a true alternative to conceptions of private property on digital networks, the question is not how can we create new nations in cyberspace, but rather, why would we?

\footnotetext{
${ }^{221}$ The term 'communities of affinity' was inspired by Primavera de Fillippi, "Citizenship in the Era of BlockchainBased Virtual Nations" in Debating Transformations of National Citizenship, ed. Rainer Baubock (Switzerland: Springer Open, 2018): 267-277.
} 


\section{Chapter 2 -Smart Contracts, Decentralized Networks, and the Commons}

"We stormed the gates, raised the flags. Just the same old story. We seized the throne, subjugate. We should have burned it to the ground."

- Rise Against

\section{The Potentials of Smart Contracts and DAOs}

While the release of Bitcoin and the underlying technology of blockchain has been monumental in conceptualizing the future of identity, the protocol that allows for the transfer of value in Bitcoin through the usage of a decentralized ledger is not limited to simply recording data. Indeed, in 2013, then 18-year-old Vitalik Buterin lamented the state of the Bitcoin blockchain as limited in its scope, which was based predominantly on the fact that creator Satoshi Nakamoto deliberately wrote the protocol in an antiquated programming language in order to improve security - though it simultaneously had the effect of limiting the complexity of possible transactions. ${ }^{22}$ In lieu of this, Buterin realized that rather than attempting to build on top of the inherently limited Bitcoin, he could write a version of the protocol using a much more accessible Turing-complete programming language (called Solidity) that would allow for far more complex and revolutionary digital services to be facilitated through a decentralized ledger. $^{223}$

In January of 2014, Buterin (and eventually a team of co-creators) did just that when they announced the Ethereum blockchain. ${ }^{224}$ The Ethereum blockchain was primarily developed to surpass the utility of Bitcoin and to 'move far beyond just currency' by serving as 'the ultimate abstract foundational layer' in the form of an 'alternative protocol' upon which anyone can

\footnotetext{
222 Robert Pybus, "The Uncanny Mind that Built Ethereum" in Wired (13 June 2016).

223 Ibid; Primavera De Filippi \& Aaron Wright, Blockchain and the Law (Cambridge: Harvard University Press, 2018). 28

224 Taylor Gerring, "Cut and dry: building a dream" in Ethereum Blog (9 February 2016).
} 
create decentralized sorts of applications. ${ }^{225}$ Accordingly, in the words of De Filippi and Aaron Wright, "Ethereum is paving the way for a new paradigm of computing — one where software applications are no longer controlled by a central authority but rather operate autonomously on a decentralized, peer-to-peer network."226

Ethereum has thus been referred to as both Bitcoin 2.0 and as the Web 3.0. ${ }^{227}$ The reasoning for this is because Ethereum serves as a general purpose and decentralized ecosystem, where Buterin explains it by comparing it to both Google's Android operating system and Apple's IOS: inside Android and IOS you can have an incredibly wide and eclectic array of applications (apps) written in a variety of programming languages, where anyone can create an app and anyone can download an app and run it. ${ }^{228}$ Ethereum essentially brings this flexibility to the world of decentralization.

It does this by allowing for the development of 'smart contracts'. A smart contract is essentially a small computer program stored in the form of data or information. ${ }^{229}$ In its simplest understanding, it allows for the creation of a set of code that operates under the parameter of 'if this happens, then I will do this.' What Ethereum facilitates is the relatively easy capability to create this small computer program and to host it as a set of data on the decentralized Ethereum ledger - which is similar in many ways to that of the Bitcoin DLT in its consensus mechanism

\footnotetext{
${ }^{225}$ Ethereum, A Next-Generation Smart Contract and Decentralized Application Platform (2015), available at https://github.com/ethereum/wiki/wiki/White-Paper.

${ }^{226}$ De Filippi \& Wright, Blockchain and the Law, 29

${ }^{227}$ Vitalik Buterin (Bitcoinist.net), “D2P3 - TNABC 2015 - VITALIK BUTERIN FOUNDER ETHEREUM - Bitcoin 2.0 - Ideas and Applications" Youtube video (26 January 2015), available at https://www.youtube.com/watch?v=FjheOMVRHO4; Ethereum, Web 3: A platform for decentralized apps (2016), available at http://ethdocs.org/en/latest/introduction/web3.html

${ }^{228}$ Vitalik Buterin (TechCrunch), "Decentralizing Everything with Ethereum's Vitalik Buterin | Disrupt SF 2017" Youtube video (18 September 2018), available at https://www.youtube.com/watch?v=WSN5BaCzsbo ${ }^{229}$ De Filippi \& Wright, Blockchain and the Law, 27
} 
and overall structure, though differing in scope and in the types of accounts that can be created. $^{230}$

Indeed, there are two types of accounts that can be created on the Ethereum network (as opposed to the one type that can be created on Bitcoin). The first type of account on Ethereum is similar to the type of account that a user can create on the Bitcoin network, which is owned by the individual user and can be used to transfer funds or accept funds from another user - though, as the previous chapter discussed, accounts created on other blockchains in the context of SSDI are capable of holding a wide variety of different data in the form of identity markers. Nevertheless, the account is directly related to a user and this type of account on Ethereum is referred to as an 'externally owned account., 231

The other type of account the Ethereum allows for is called a 'contract account,' which is used for smart contract applications and which has earned it the designation of Bitcoin 2.0. A contract account is essentially the aforementioned small computer program that is publicly available for all to see but is not owned or controlled by any individual user (though it can be created by any user or organization). Effectively, a contract account is 'owned and controlled' by the governing set of code that the users creates and therefore it simply abides by the set of parameters initiated within that code. ${ }^{232}$ Thus, with a contract account, any time it receives relevant information or the parameters of the code are met, it activates itself and sets off to do whatever the code programmed it to do.

\footnotetext{
230 lbid., 28

231 Ibid., 28

232 Ethereum, A Next-Generation Smart Contract and Decentralized Application Platform (2015).
} 
Importantly though, "contracts in Ethereum should not be seen as something that should be 'fulfilled' or 'complied with'; rather, they are more like 'autonomous agents' that live inside of the Ethereum execution environment, always executing a specific piece of code when 'poked' by a message or transaction." ${ }^{, 233}$ In this instance, a 'message' is characterized as an object containing 'the sender of the message; the recipient of the message; the amount of ether to transfer alongside the message; and an optional data field. ${ }^{234}$ Thus, the message is essentially an accruement of pertinent information needed to set a contract into order and they are generated by contract accounts. A transaction, on the other hand, is essentially the same as a message with the main difference being that it is initiated by an externally owned account. ${ }^{235}$

The autonomous agents in the form of smart contracts are activated and processed by the Ethereum Virtual Machine (EVM). ${ }^{236}$ The EVM can effectively be thought of as a global and decentralized computer that 'runs' all of the 'small computer programs'; indeed, the EVM is sometimes referred to as a 'world computer'. ${ }^{237}$ In this, every node that makes up the decentralized ledger that is the Ethereum blockchain not only hosts the information and data in the form of code that is a small computer program, but each node actually executes and runs the program. ${ }^{238}$ Thus, the entire system is effectively a global autonomous operating system that powers computing programs; in the words of De Filippi and Wright, "The open and decentralized nature of Ethereum allows smart contracts to be deployed pseudonymously and to operate in a largely autonomous manner. Because all active nodes on Ethereum run the code of

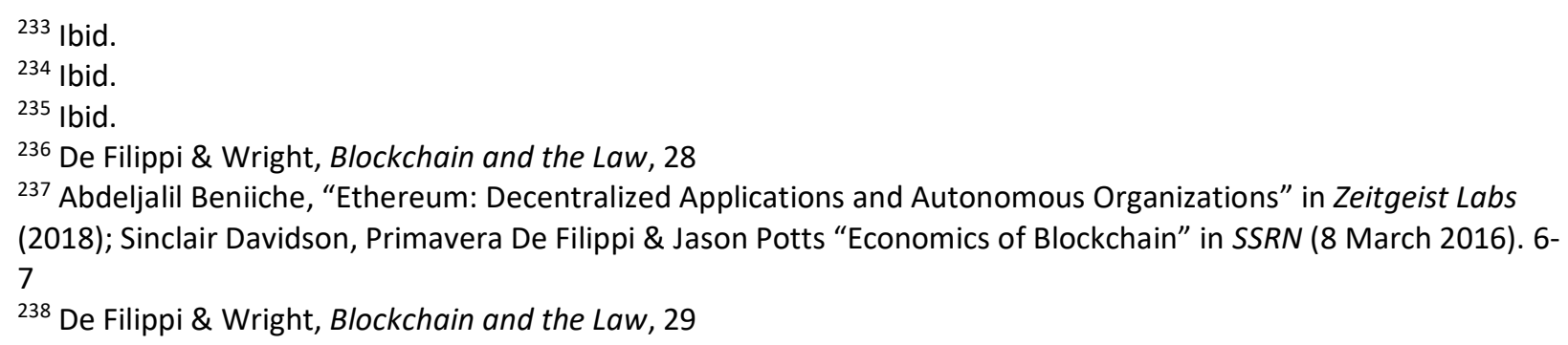


every smart contract, the code is not controlled by — and cannot be halted by — any single party." 239

Important to know about smart contracts, however, is that through 'messages', one autonomous smart contract can be used to initiate another, which can be used to generate another, and so on. Thus, though each smart contract can be considered as a 'small computer program', being that they communicate and feed off one another, a collection of these small computing programs that initialize one another can be considered as a rather comprehensive computer program, and a very large collection of these small autonomous agents feeding off of each other can operate rather complex applications; what is an application if not a collection of simple interacting protocols operating together towards a general purpose? Here, we are starting to see Buterin's vision of a decentralized operating system running decentralized protocols that accumulate into what are referred to as decentralized applications (dApps - though it is important to note that dApps refer to decentralized applications on the blockchain as opposed to peer-to-peer decentralized applications such as BitTorrent).

Though these sorts of projects are still very much in their infancy, a dApp can theoretically, of course - replace any centralized web application that is hosted on centralized servers. Examples of centralized applications are essentially endless, but some of the more prominent ones already mentioned throughout earlier chapters include Facebook, Amazon, and Google. Again, it is important to remember that this is theoretical being that there are countless hurdles and obstacles in making this claim a reality; nevertheless, it would theoretically be possible to create these services through a dApp, where all of the data and computing was

${ }^{239}$ De Filippi \& Wright, Blockchain and the Law, 29 
distributed amongst all of the nodes that make up the network. Effectively, the decentralized Facebook, for instance, would be operated by all of the nodes that make up the network and therefore none of them would have full operating power. ${ }^{240}$

Regarding dApps, former C.O.O. of Ethereum Stephan Tual describes them as "applications that have no middle men, where users interact with social systems, financial systems, gaming interfaces - all in a peer to peer fashion. Because the Ethereum network will be distributed on hundreds of thousands of computers around the globe, this all takes place on a censorship-proof foundation." ${ }^{241}$ When conceptualizing a dApp, then, it would perhaps be easiest to think of it as a an open source protocol completing a particular function without any ownership - short of the collection of nodes that make up the network who operate in a form of decentralized consensus facilitated by a blockchain. A Facebook without a Mark Zuckerberg or executive board could be used as a metaphor: just a social media website that operates autonomously without ownership and is maintained by a large collection of autonomous nodes. Bitcoin can be used as an actual example of a dApp, however, being that it is an open-source network that performs a function while not being owned by any individual or group, where the collection of nodes operate on a consensus mechanism.

Any individual or organization can create a dApp for any purpose they desire, but an important aspect that has so far been left out of this analysis is the question of why would an individual or organization go through the effort, and why would a node dedicate computing power to the functioning of a dApp? The answer to this can be found in one other key aspect of a dApp as identified by one of the most prominent organizations for identifying and ranking the

\footnotetext{
240 Beniiche, Ethereum: Decentralized Applications and Autonomous Organizations.

${ }^{241}$ Ethereum, "What is Ethereum?" Youtube Video (4 June 2014), available at

https://www.youtube.com/watch?time_continue=82\&v=Clw-qf1sUZg
} 
thousands of existing dApps: stateofthedapp.com. In this, the organization asserts that a dApp must use a cryptographic token and must also generate tokens. ${ }^{242}$

Cryptographic tokens are - for the most part - generated on every blockchain that has come into existence since the release of Bitcoin, and a 'bitcoin' in itself is an example of a cryptographic token (or a crypto-currency as it is also referred to - though some consider there to be variations between the two). The way that crypto-tokens are generated on the Bitcoin network (how bitcoins are generated) is through the aforementioned PoW mechanism in a process called 'mining' - and this process also performs the function of securing and maintaining the network. To mine bitcoins, a user would set up a node which is essentially a computer (or computing power) dedicated to securing and maintaining the Bitcoin network.

Understanding mining is to understand that every transaction that occurs on the Bitcoin network is accumulated into 'blocks' which is roughly a ten-minute timestamp. Thus, every ten minutes a new block is created which designates all of the transactions in the old block as officialized/notarized and part of the immutable ledger that is the chain of blocks. In order to do this, every node on the network is racing against one another in order to be the one to create the new block; the creator of the new block is rewarded with a certain amount of bitcoin as well as all of the transaction fees from the former block. In order to create a new block, the node must 'solve' a mathematical puzzle - which is effectively a randomly generated trial and error computation - and the 'miner' who solves it gets the reward associated with the creation of a new block. Once a miner solves the equation, they broadcast it to the network where other nodes can do a simple calculation to verify that it is legitimate. Once this occurs, the former block is

${ }^{242}$ State of the Dapps, What's a Dapp? (2019), available at https://www.stateofthedapps.com/whats-a-dapp. 
added to the chain and miners race to create the next block - which takes roughly ten minutes for a winner to emerge being that the Bitcoin protocol adjusts the difficulty of the equation based on how many miners are in the race. ${ }^{243}$

This process of mining provides a financial incentivization for the nodes who upkeep the network, and many of the other blockchains that have emerged use the same or at least a very similar protocol - including Ethereum (though Ethereum's timestamp is closer to ten seconds as opposed to the ten minutes of Bitcoin). Miners of Ethereum are rewarded for the upkeep of the network in Ethereum's native crypto-currency which is called Ether, though it should be noted at this point that Ethereum is working towards the implementation of another consensus mechanism called Proof of Stake (PoS). While the functioning of Proof of Stake is not entirely relevant to this analysis, it is representative of alternatives to the PoW model and it should be said that many, many blockchains are experimenting with alternative/improved models to that of Bitcoins PoW (the significance of this will become clear in the subsequent sections).

Returning to dApps on the Ethereum network though, nearly all of them produce their own cryptographic token (through whatever kind of consensus mechanism) and these tokens are generally awarded to those who upkeep the network. Tokens hold an inherent value and can be traded on a variety of digital markets for other crypto-currencies, or they can even be turned into a more standard national currency. Generally speaking, tokens built on top of the Ethereum network and that are thus compatible with the Ethereum platform are referred to as ERC-20 compliant. $^{244}$

\footnotetext{
${ }^{243}$ De Filippi \& Wright, Blockchain and the Law, 24.

${ }^{244}$ Maxwell William, "ERC-20 Tokens Explaine" in CoinTelegraph (12 May 2018), available at https://cointelegraph.com/explained/erc-20-tokens-explained.
} 
Conceptualizing the hypothetical dApp Facebook then (though we will turn to actual examples in the following section), the decentralized social media platform could be created and placed onto the Ethereum platform where those who participate in the computing of the smart contracts that make up the sophisticated application would be rewarded in an ERC-20 compliant crypto-currency - which they could then either use to navigate other Ethereum based dApps or they could trade in for either another crypto-currency or perhaps a national currency.

However, while this in itself might be enticing enough for people to host the network (and it really has been for many blockchains including Ethereum), just hosting a fragment of the computing power is not the only means by which people could be issued the cryptographic token. Indeed, included in the capabilities of smart contracts and dApps is the ability to issue native tokens for a very wide and eclectic array of services, so that active participation on the network could perhaps result in forms of payment issued autonomously and automatically via smart contracts. Thus, conceptualizing a dApp involves the realization that the dApps not only reward their users for hosting the network, but could potentially reward the users for improving the networks (which can sometimes occur just by using it).

Being that the dApp generates and distributes the tokens via an automatic and predetermined set of code though - and that users can be rewarded automatically via their improvement of the network or active participation in it - it must be realized that what we are describing should no longer just be considered as just a decentralized application. Going back to the example of the hypothetical dApp Facebook; the dApp is simply a collection of smart contracts that operate as a hypothetical social media platform. However, the pre-written code cannot do everything - a social media platform cannot really exist without users, for instance. Thus, the set of code could perhaps automatically reward users just for participating on the 
network. However, other things that the dApp cannot do is update the network which would make it dependent on humans to write new code. In order to facilitate this, the dApp could automatically outsource the tasks to whomever is willing to do it and automatically reward the user upon the task's completion. In this conceptualization, however, the set of contracts has seemingly moved beyond a simple application and they are effectively organizing individuals under a common objective in a decentralized and autonomous manner - what blockchain enthusiasts refer to as a decentralized autonomous organization (DAO).

Buterin himself refers to DAOs as a sort of 'holy grail' of blockchain development, describing them simply as "an entity that lives on the internet and exists autonomously, but also heavily relies on hiring individuals to perform certain tasks that the automaton itself cannot do." 245 Buterin continues to say that they must contain "some kind of internal property that is valuable in some way, and it has the ability to use that property as a mechanism for rewarding certain activities." While this as a principle can perhaps be simple to understand, the implications of it simply cannot be overstated.

Indeed, DAOs are essentially comprehensive collections of smart contracts and dApps that are used to manage an entire organization through code and without human management; in the words of Buterin, DAOs are "long-term smart contracts that contain the assets and encode the bylaws of an entire organization." 246 In this, DAOs effectively decentralize power within an organization by eliminating hierarchical structure, where sets of code becomes the means by which individuals are able to collaborate together towards a greater goal. Maintenance of the

\footnotetext{
${ }^{245}$ Vitalik Buterin, "DAOs, DACs, DAs and More: An Incomplete Terminology Guide" in Ethereum Blog (6 May 2014), available at https://blog.ethereum.org/2014/05/06/daos-dacs-das-and-more-an-incomplete-terminologyguide/.

${ }^{246}$ Vitalik Buterin, cited from JZ Garrod, "The Real World of Decentralized Autonomous Society" in TripleC (14:1, 2016). 65
} 
network is done by consensus, and though we have only really described PoW, decisions requiring human intervention on a DAO are generally done in a democratic and consultative manner. ${ }^{247}$ Thus, a DAO is effectively owned by all of the nodes that make up the network and the network allocates resources in a democratized and automatic manner. Going back to the example of the decentralized Facebook, one way to conceptualize it would be that the DAO Facebook would be owned and operated by all of the digital identities that make the platform an obviously revolutionary notion.

\section{The Realizations of Smart Contracts and DAOs}

While it should be again stated that the development of blockchain 2.0, dApps, and DAOs are still very much in their infancy (being that the technology only really came to fruition in 2015), many individuals and organizations have come to the forefront of the discussion envisioning the near-endless applications of DLT and its potentials to revolutionize whatever industry to which it can be applied. Further, many are beginning to conceptualize the broader grandiose implications of the technology, particularly with the realization that dApps and DAOs represent a new paradigm for social organization at nearly every level - from the firm to the institution and from the local to the global.

Starting at a more basic and pragmatic level, however, and as previously mentioned, a dApp or a DAO could theoretically replace most centralized organizations that exist today. Turning back to the hypothetical decentralized Facebook from the previous section, for instance;

\footnotetext{
247 Ying-ying Hsieh \& Jean-Philippe Vergne, "Bitcoin and the Rise of Decentralized Autonomous Organizations" in SSRN (11 December 2017). 2
} 
though there is some controversy regarding whether or not it is a fully decentralized organization, there is one particular blockchain-empowered platform that exists today that illustrates how a dApp or a DAO Facebook could effectively operate and which has inspired others to replicate the model on alternative blockchains for alternative purposes.

Indeed, one of the more popular platforms in the blockchain community is that of Steemit, which is an application that exists on the Steem blockchain. The Steemit whitepaper describes itself as "a blockchain database that supports community building and social interaction with cryptocurrency rewards." 248 As a platform, it could most easily be understood as an autonomous network where users can write and upload blog posts to share on whatever topic they deem worthy. Additionally, Steemit also employs a 'Reddit-like' organizational algorithm that also allows users to 'upvote' stories that they like which simultaneously has the effect of curating stories within home feeds based on popularity. Finally, its most unique (and some would argue revolutionary) feature is that Steem effectively rewards its users for the creation of valuable content. ${ }^{249}$

It does this through the issuance of a native crypto-currency called Steem Dollars or SBD (for Steem Blockchain Dollars), and the application issues SBD automatically and algorithmically under the premise of three key principles. ${ }^{250}$ The first principle is "Everyone who contributes to a venture should receive pro-rata ownership, payment, or debt from the venture." 251 The second principle is that "All forms of capital are equally valuable. This means that those who contribute their scarce time and attention toward producing and curating content

\footnotetext{
${ }^{248}$ Steemit, Steem: An incentivized, blockchain-based, public content platform (June 2018), available at https://steem.com/steem-whitepaper.pdf. 2

249 lbid., 5

250 lbid., 5-8

251 Ibid., 5
} 
for others are just as valuable as those who contribute their scarce cash." ${ }^{" 252}$ Finally, the third principle is "that the community creates value to serve its members." 253

Practically, what this looks like is Steemit rewards the members of the Steem community with a crypto-currency based on their very participation on the network - or as Steem puts it, "everyone's meaningful contribution to the community should be recognized for the value it adds." ${ }^{254}$ Users are thus paid for the creation of popular content (recognized by the community through upvoting) in $\mathrm{SBD}$, and this process is again done automatically via algorithm; the more 'likes' you get the more 'dollars' you get. In addition, users can be 'paid' in SBD by commenting something valuable on another's post (as recognized through upvoting) or even just by voting on another's post. ${ }^{255}$ Finally, Steemit also employs a form of reward based on a user's ongoing participation within the network called 'Steem Power' (SP). ${ }^{256}$ In this, a user's participation on the network also distributes them SP, and a user's "influence over the distribution of the rewards pool is directly proportional to the amount of SP that they have."257 Thus, the more popular somebody is on the Steemit network, the more valuable their opinion is in voting.

One of the more interesting organizations to adopt a model similar to Steemit is that of Sapien, which is a decentralized social news platform that is ERC-20 compliant and built on the Ethereum network. ${ }^{258}$ It describes itself as "a democratized, tokenized, reputation-based social

\footnotetext{
252 Ibid.,

253 lbid.,

${ }^{254}$ Ibid., 6

255 Ibid.

${ }^{256}$ Ibid., 9

${ }^{257}$ Ibid., 7

${ }^{258}$ Sapien, Sapien: Decentralized Social News Platform (March 2018), available at https://www.sapien.network/static/pdf/SPNv1_3.pdf.
} 
news platform capable of rewarding millions of digital content creators without any centralized intermediaries." 259 Sapien similarly rewards participation in the network autonomously via posting, commenting, and voting, but also implement what they refer to as a Democratized Autonomous Platform (DAP), where holders of the native Sapien cryptocurrency (SPN) will "be able to participate in DAP voting to help influence the company roadmap and organization." 260 Thus, decisions regarding the development of the organization are done democratically by the users of the network.

Another ERC-20 compliant organization built on top of the Ethereum blockchain is Refereum, and just like Sapien and Steemit, it is looking to decentralize a digital industry and allocate the value generated within it to the users who create the value. In this example, however, Refereum is focusing on the gaming industry and looking to reward gamers for their engagement and purchases on the network with its native RFR token. ${ }^{261}$ In 2018 , Refereum reached over 1 million users and had distributed over a million dollars in rewards. ${ }^{262}$ Further, the organization has been endorsed by some of the world's largest gaming communities in Twitch and Unity, who have collaborated with Refereum to allow for its participation on their platforms. ${ }^{263}$ Finally, Refereum can already be used for some of the world's most popular games, such as Fortnight and Battlefield $V .^{264}$

\footnotetext{
259 lbid., 6

260 lbid., 37

${ }^{261}$ Refereum (White Paper), Refereum: Referrals in the blockchain (25 January 2018), available at https://old.refereum.com/info/whitepaper. 2

262 Refereum, "1 Million Refereum Users | 2018 Year End Review" in Medium (21, December 2018), available at https://medium.com/refereum/1-million-refereum-users-2018-year-end-review-21397ad9be19.

263 Refereum, Referrals on the Blockchain, 19-20.

264 Refereum, 1 Million Users.
} 
Through Refereum, game developers can purchase RFR tokens and then automatically distribute them to anyone who is participating in the game's eco-system for whatever reason they deem worthy via smart contracts. ${ }^{265}$ A gamer, for instance, can be issued 'points' just by purchasing a game, which can then be used to purchase more games. ${ }^{266}$ In addition, an influencer can be distributed RFR based on gamers watching them play. Even just playing a game can issue a user this form of currency, where hitting an achievement can unlock a certain amount of 'money' that can easily be used for purchases in the Refereum marketplace (predominantly games and game-related features). ${ }^{267}$

What these organization are effectively doing is rewarding users for the creation of value on their network based simply on their participation on the network. This, however, is not the only means by which blockchain can revolutionize digital networks. While there are many instances where users create values on a network based on their participation in it (predominantly seen through social media platforms), there are many other examples of value creation where users are merely utilizing centralized networks and applications as a means of digitally connecting.

This example is perhaps most apparent in the music industry, where centralized companies like Apple Music, Google Play, Spotify, Soundcloud, Pandora, and so on have effectively monopolized the streaming of digital music in the West and decide by their own prerogative how to allocate the value that is created on these networks. Perhaps as to be expected, these companies are somewhat infamous for not paying nearly a sufficient value to

\footnotetext{
265 Ibid., 13

266 Bitcago Crypto, "How Referum Works" Youtube Video (2 February 2018), available at https://www.youtube.com/watch?v=316VclIRRS8.

267 Refereum, Earn Rewards for Playing and Sharing Games, available at https://refereum.com/games
} 
music creators while all of the wealth that is generated via the distribution of the artists' tracks is accumulated at the top of the organization that distributes it rather than in the hands of those who actually create the music.

There have been many blockchain enthusiasts who have recognized this as an issue worthy of addressing, however, and have since entered the realm by employing smart contracts and dApps as the primary means of distributing and allocating the value that is accumulated on the network. One of the larger and more established organizations is that of Choon, who at the time of writing host over 11 thousand artists on their network and over 40 thousand tracks. ${ }^{268}$ Choon is in the process of developing a 'trustless, low-cost decentralized marketplace for music' where artists are compensated for users streaming their tracks in a native ERC-20 compliant cryptocurrency called 'Notes'. ${ }^{269}$ This organization already has a working beta version, and it compares itself to both Spotify and Soundcloud in that it seeks to be a "commercial-grade streaming service with web and mobile clients running on the Internet." 270 What makes it different, however, is that Choon employs what it refers to as 'Smart Record Contracts' that "allow the various stakeholders of a piece of music to record their ownership shares in an indisputable contract that cannot be amended unless all parties agree to it. Furthermore, Smart Record Contracts can then be used to distribute NOTES royalties due for streaming in real time to multiple artists' wallets." ${ }^{271}$

More practically, what this looks like is that every time a user streams an artist's song, the artist will automatically be distributed Notes via Choon's algorithm - which can then be

\footnotetext{
${ }^{268}$ Choon, Crypto \& Tunes, available at https://choon.co/about.

${ }^{269}$ Choon (White Paper), Choon: The Blockchain's Music Solution (2017), available at https://www.choon.co/public/pdf/choon_white_paper.pdf.

270 lbid., 16

271 lbid., 11
} 
divided automatically amongst the creators of the music in whichever way they see fit: $40 \%$ for the singer, $30 \%$ for the guitarist, $20 \%$ for the drummer, and $10 \%$ for the song writer, for example. ${ }^{272}$ These Notes are generated automatically via the dApp's algorithm (similar to the ways in which Bitcoin is generated), and Choon will distribute Notes in what it deems to be a fair and transparent pro-rata process based on the amount of streams one has: more streams means more notes. ${ }^{273}$ In this way, artists who have uploaded music are effectively acting as 'miners' in what is referred to as 'Streaming as Mining., 274

While Streaming as Mining is the first and most predominant for launch, they also plan to employ revenue based on advertising, revenue based on subscriptions, and revenue based on direct payments. ${ }^{275}$ In regards to the more interesting subscription models though, users will pay a monthly subscription fee similar to models like that of Spotify, with the key difference that though they will be able to purchase their subscription in traditional fiat currencies such as the American dollar, it will then be converted 'under the hood' by the organization to Notes. ${ }^{276}$ Upon this conversion, the user's subscription will then be distributed proportionally to all of the artists that generated the music that they streamed; a user's subscription fee will be funneled to the artists they actually listened to based on how much they actually listened to the artist. ${ }^{277}$ Further, the platform will also allow the user to see how much of their monthly contribution went to each of their listened-to artists. ${ }^{278}$ Finally, the platform also allows for direct payments which

\footnotetext{
${ }^{272}$ Choon, "Introducing Choon: Helping Artists Get Paid Fairly" Youtube Video (20 November 2017), available at https://www.youtube.com/watch?v=ruy3H1IPhEE

273 Choon, The Blockchain's Music Solution, 6

274 Ibid.

${ }^{275}$ Choon, The Blockchain's Music Solution, 8

276 Ibid.

277 lbid.

278 Ibid.
} 
gives users the opportunity to pay artists directly for songs, can allow them to tip, can give them the opportunity to pay directly for live shows and collectibles, and so on. ${ }^{279}$

There are many other organizations that are working towards the decentralization of the music industry, and Choon just happens to be one of the more prominent and developed ones. Further, being that they also have also declared that they will being accepting fiat currency for subscription payments, they have allowed ample room for mainstream adoption that is not dependent on one's engagement with the blockchain world (which is still hidden from most). However, what could perhaps be considered the most interesting aspect of their organization (at least in this writer's mind) is that they hypothesize on a near future where the possibilities for innovation are nearly endless, focusing on two primary examples. The first is where "a group decides to become a fan-owned collective, where any fan can join the Smart Record Contract, which distributes NOTES equally across all fans." ${ }^{280}$ The second is "an artist crowd-funds her album using NOTES, giving all funders a profit split in the resulting musical release."281 In this, it would seem that they are starting to envision what would more closely resemble a DAO.

Regarding DAOs, then; one of the more recognizable organizations worthy of mentioning in this field is Consensys, which was developed by Joseph Lubin who is recognized as one of the co-founders of Ethereum. Consensys can best be understood as a sort of umbrella organization that fosters dApps and DAOs on the Ethereum blockchain through the provision of expertise and resources. ${ }^{282}$ They describe themselves as "A global formation of technologists and

\footnotetext{
279 Ibid.

280 lbid., 11

281 Ibid.

282 Consensys, About Us (Consensus Systems, 2019), available at https://consensys.net/about/.
} 
entrepreneurs building the infrastructure and applications that will enable a decentralized future." 283 Further, they claim that they are at the 'forefront of a revolution. ${ }^{284}$

In this, Consensys have established some fairly significant partnerships, and their development lab has incubated over 50 premier projects on the Ethereum network (including that of uPort described in the previous chapter). These projects, in the words of Joseph Lubin, are building "everything from infrastructure protocols, developer tools, core components; to protocol-based open-platform network applications. These activate the technologies that will impact every industry and shape the future." ${ }^{285}$ These projects cover a vast array of different fields, including the aforementioned uPort; Civil, which is a blockchain empowered 'community-owned journalism network based on transparency and trust;' ${ }^{286}$ Openlaw, 'a userfriendly, legally compliant blockchain-based protocol for the creation and execution of smart legal agreements; ${ }^{287}$ the highly-popular Metamask, which allows a user to run Ethereum dApps on their browser; ${ }^{288}$ Circles, which has the aim to create and distribute a globally accessible universal income; ${ }^{289}$ and many, many more. They have also teamed with governments such as Dubai and presented at the World Governance Forum.

While Consensys has thus been fostering many real-world applications and has been engaged in many practical developments, it is how it views the nature of organization in itself and how it claims to be embodying it - that perhaps makes it worthy of the title 'at the forefront

\footnotetext{
283 Ibid.

284 Ibid.

285 Ibid.

${ }^{286}$ Civil, Stories that Need Telling (The Civil Media Company, 2019), available at https://civil.co/.

287 Open Law, Building the Future of Law, One Legal Agreement and Contract at a Time (Aaron Wright, David Roon, and ConsenSys AG, 2019), available at https://www.openlaw.io/.

288 Metamask, Brings Ethereum to your browser. Available at https://metamask.io/.

${ }^{289}$ Circles, "Fair Money for All: Basic Income on the Blockchain" in Wordpress (16 February 2017), available at https://ourbasicincome.wordpress.com.
} 
of a revolution'. In this, Consensys refers to its organizational structure as representative of an 'organism,' with Lubin claiming that they "take this decentralization thing very seriously, in all of our work. And in how we organize ourselves. Our teams are highly autonomous, but we communicate richly [and] we work collaboratively." ${ }^{290}$ They claim that they do not have a monolithic master brand, and instead consider themselves as a network of peers working autonomously and asynchronously yet staying accountable to one another. ${ }^{291}$

To ensure that they remain a flat and non-hierarchical organization, they utilize what they call the Traditional Management Nullification Tool (TMNT). Users can integrate to the TMNT system via their uPort SSDI, and once they are within the system, it allows the facilitation of all of the different relationships and different responsibilities a user can opt into via what the organization(s) deem worthy of being done and calls out for. ${ }^{292}$ The structures are held together via the legal smart contracts developed by Openlaw, and according to Lubin, "These mechanisms enable agreements to be formed and signed, you can sequence different actions, you can send money into a contract, escrow money in the contract, pay money out of the agreement, and send data into a service level agreement, get paid out based on the data and based on the performance of that particular team."

Essentially, what Consensys is in the process of creating is a new vision of a nonhierarchical and decentralized organization, where the structure is held together by 'long-term smart contracts that contain the assets and encode the bylaws of an entire organization' - as envisioned by Butalik in his description of the 'holy grail.' They are not the only ones who

\footnotetext{
${ }^{290}$ ConsenSys Media, "OE LUBIN - Nature Of The Firm, v2.0 KEYNOTE from EtherealNY \#Blockchain Conference 2018" Youtube Video (13 May 2018), available at https://www.youtube.com/watch?v=SQbcGhnv4jw. ${ }^{291}$ Consensys, "Consensys Monthly Report - October 2018" in Medium (31 October 2018), available at https://media.consensys.net/consensys-monthly-report-october-2018-7f8bae68842b.

${ }^{292}$ ConsenSys Media, Joe Lubin - Nature of the Firm.
} 
recognize the potentials of this new form of organization, however, and they are not the only who are fighting to enable it.

Another prominent organization in the world of dApps and DAOs is that of Aragon, who describe their opt-in network as 'The world's first digital jurisdiction. ${ }^{.293}$ In this, what the organization is looking to do is create the tools for decentralized organizations to thrive by making it easier to create the smart contracts that encode the bylaws of the organization; in the words of the organization's white paper, "The Aragon Network provides a subjective governance layer that improves the overall usability of Ethereum by providing a mechanism for pseudoanonymous blockchain entities, including [DAOs] and individuals, to create flexible humanreadable agreements that are enforceable on-chain." ${ }^{294}$

In this, the Aragon organization recognizes the nuances within the intersection of digitally encoded contracts as compared to legally binding agreements between individuals within an organization, and what they are proposing is what they refer to as an 'Aragon Agreement': a set of smart contracts and a human readable document that is cryptographically signed, which specifies 'the responsibilities of relevant agents, their respective liabilities, and what mechanism will be used to enforce the agreement in the event of a dispute. ${ }^{295}$ Individuals participating in a DAO would thus be able to easily agree-upon how the organization should operate (including everything from responsibilities to distribution of generated value), and upon this agreement they would be able to set the organization about by employing autonomous code that not only operates and holds the organization together, but has all of the mechanisms for

\footnotetext{
${ }^{293}$ Aragon, The World's First Digital Jurisdiction, available at https://aragon.org/network/.

${ }^{294}$ Aragon (White Paper), Aragon Network, available at https://github.com/aragon/whitepaper

295 Ibid.
} 
resolving disputes - including digital courts, juries, adjudication processes, and arbitrations. ${ }^{296}$ While this as a technology in itself is revolutionary, it is the envisioned implications of the organization that truly make Aragon awe-inspiring.

\section{Networks and the Commons}

Much in line with the organization Bitnation and also seemingly influenced by the notions of the crypto-anarchists and cypherpunks, Aragon is not shy about declaring its dissatisfaction with contemporary political and economic systems while looking to develop the technology to help transcend them. In their manifesto, they state that they believe that "the fate of humanity will be decided at the frontier of technological innovation... [that] we should use technology as a liberating tool to unleash all the goodwill and creativity of our species, rather than as a tool to enslave and take advantage of one another."297 They assert that "Aragon is a fight for freedom,' recognizing that the provision of self-sovereign identities only goes so far and that there is still the need for a decentralized and non-hierarchical organization of sovereign individuals that simultaneously defends self-sovereignty. ${ }^{298}$

Much like the crypto-anarchists of old, Aragon recognizes that governing organizations should be free from coercion both within and by outside forces, arguing that "Decentralized organizations change our relationship with governance: from something that is imposed upon us by others, into something we choose to opt into. Where we are equally serving and served, rather than just serving... Sovereign individuals will be able to freely express themselves and transact with each other without any kind of intermediary exercising their unjustified power and

\footnotetext{
296 Ibid.

297 Aragon, The Aragon Manifesto (8 May 2018), available at https://blog.aragon.org/the-aragon-manifesto4a21212eac03/. 298 Ibid.
} 
oppressing them." 299 Finally, they recognize that "Choices can only be made freely in the absence of coercion and extortion... We must strive to create systems in which a large number of diverse stakeholders have a say, in order for common goods to be responsibly governed by their communities."

This notion of how an organization of individuals should operate directly challenges one of the primary theories within the economic institutions of capitalism: that of private property and the firm as a self-enclosed system operating within a capitalist free market. ${ }^{300}$ This challenge, in turn, seemingly becomes representative of an alternative to the entirety of the underlying justifications and rationalizations of a capitalist free market - which brings forth questions regarding the necessity of 'The Sovereign'. In order to conceptualize this, however, the firm (i.e. private property) must be contextualized within the system as a whole. Regarding this, then; the capitalist economic system is premised on the notion that the capitalist society simply 'works itself' via a market; according to Sir Arthur Salton, "For its current operation it is under no central control, it needs no central survey. Over the whole range of human activity and human need, supply is adjusted to demand, and production to consumption, by a process that is automatic, elastic and responsive." ${ }^{301}$ R.H. Coase - long predating Lubin's simile - argues that a society guided by a price mechanism becomes 'not an organization but an organism. '302

Under the capitalist purview, this is desired because markets are seen as the only method that can guide a free society. Indeed, Milton Friedman posited in the aforementioned Capitalism and Freedom that free market exchange was the only means by which a free society could bring

\footnotetext{
299 Ibid.

300 Sinclair Davidson, Primavera De Filippi \& Jason Potts, "Blockchain and economic institutions of capitalism" in Journal of Institutional Economics (14:4, 2018). 641

${ }^{301}$ Sir Arthur Salton, cited from R.H. Coase, "The Nature of the Firm" in Wiley Online Library (November 1937). 387 302 Ibid.
} 
about "co-ordination without coercion, ${ }^{303}$ arguing that "the basic problem of social organization is how to co-ordinate the economic activities of large numbers of people." ${ }^{304}$ In his quest to find a system most agreeable with his particular conceptions of freedom, this was vital because "Political freedom means the absence of coercion of a man by his fellow men. The fundamental threat to freedom is power to coerce... by removing the organization of economic activity from the control of political authority, the market eliminates this source of coercive power." ${ }^{305}$

It is because of this ideology that figures such as Hayek and Friedman advocated for the limited role of the government in the regulation of the market. Indeed, capitalism posits the individual's sovereignty as the 'ultimate entity in the society' and that the price mechanism and the forces of competition in the division of labour are the most natural and effective means of coordinating the affairs of individuals toward a common end. ${ }^{306}$ The relevant question in this argument, then, is why is it that the price mechanism does not coordinate the inner structures of an organization or firm? Rather than this, firms predominantly operate under the hierarchical structure of the owner of the firm effectively ordering everyone around. It would seem, then, that the capitalist who sees the market as the purveyor of freedom must turn a blind eye to the operations of the firm if the on-looker is hoping to remain consistent in their conviction; as the Nobel-winning economist Coase puts it, "Within a firm, these market transactions are eliminated and in place of the complicated market structure with exchange transactions is substituted the

\footnotetext{
${ }^{303}$ Friedman, Capitalism and Freedom, 13

304 Ibid., 12

305 Ibid., 15

${ }^{306}$ Ibid., 3; Hayek, The Road to Serfdom, 13-14
} 
entrepreneur-coordinator, who directs production." "307 In fact, Coase argues that 'the distinguishing mark of the firm is the supersession of the price mechanism. ${ }^{308}$

In his seminal 1937 paper The Nature of the Firm, Coase looks to provide a neoclassicaleconomic justification for the emergence of a hierarchical division of labour seen within the firm, when theoretically, a price mechanism meant to coordinate without coercion should be able to manage the firm internally. ${ }^{309}$ To provide this justification, Coase references Professor Knight when he states that the inevitability of uncertainty within the firm leads to higher transaction costs, which has the effect of negating external market forces: "When uncertainty is present and the task of deciding what to do and how to do it takes the ascendancy over that of execution [of] the internal organization [sic]...Centralization of this deciding and controlling function is imperative, a process of 'cephalisation' is inevitable."310

Under the precipice that the price mechanism and the market are the most natural and universal means of coordinating human affairs, the logic is that a firm must remain hierarchical in order to properly participate in the ebb and flow of the 'invisible hand' (to borrow phraseology from Adam Smith) - a 'master and servant' or 'employer and employee' relationship, as Coase refers to it. ${ }^{311}$ What is missing from this analysis, however, is the realization that it is operating under what could (and should) be considered as fairly problematic assumptions; one of the more prominent ones being that the market is something that is naturally occurring.

\footnotetext{
${ }^{307}$ Coase, The Nature of the Firm, 388

308 Ibid., 389

309 Ibid.

310 Ibid., 399

311 Ibid., 403-4
} 
As Karl Polanyi recognized in The Great Transformation, the concept of a market as a natural phenomenon is simply misguided ideology dependent on erroneous assumptions propagated by those seeking to provide the justifications for the developing institutions. ${ }^{312}$ The logic of the natural market stems from Adam Smith's recognition that humans have a tendency to truck, barter, and exchange things, and because human beings are rational, they seek to get things in the most efficient means possible; hence, left to their own devices, something like a free market will emerge - or so the argument goes. ${ }^{313}$ Anthropologist David Graeber is correct to point out, however, that for $99 \%$ of human history, nothing like this emerged, and further states that

The beauty of Polanyi's book is that it demonstrates just how completely wrong that common wisdom is. In fact, the state and its coercive powers had everything to do with the creation of what we now know as 'the market' - based as it is on institutions such as private property, national currencies, legal contracts, credit markets. All had to be created and maintained by government policy. The market was a creation of government and has always remained so. ${ }^{314}$

Indeed, Polanyi recognized that "Where markets were most highly developed, as under the mercantile system, they throve under the control of a centralized administration which fostered autarchy both in the household of the peasantry and in respect to national life. Regulation and markets, in effect, grew up together. The self-regulating market was unknown." 315

Thus, per Polanyi, the fetishization and formalization of the market as something natural and universal rather than something that is simply created and maintained by the centralized powers of state policy is as egregious as it is naïve. Without the political authority of the state,

\footnotetext{
312 David Graeber, Toward and Anthropological Theory of Value (New York: Palgrave Macmillan, 2001). 10

313 lbid.

314 Ibid.

${ }^{315}$ Karly Polanyi, The Great Transformation: The Political and Economic Origins of Our Time (Boston: Beacon Press Books, 1944). 71
} 
there would simply be no market as it is understood today - a market that is premised on institutions such as private property, national currencies, legal contracts, credit markets, and so on and so forth, and all of which must be developed and protected. In this, the protection of these institutions is dependent on the jurisprudence of the state, requiring laws and an enforcement agency should somebody transgress upon these laws; the police and the judicial system are able to physically coerce the population with the threat of violence to abide by the laws of the state, and the laws of the state are predominantly developed for the protection of the institutions of capitalistic markets. Thus, when Friedman suggests that "the fundamental threat to freedom is power to coerce... by removing the organization of economic activity from the control of political authority, the market eliminates this source of coercive power" - it should simply be considered oxy-moronic. The capitalist market could not exist without coercive power of the state's political authority because its very institutions are dependent on its protection.

Of the institutions mentioned, it is indeed that of private property that has most to do with the development of a capitalistic free market dependent on the state's jurisprudence for protection - the institution that is somewhat glaringly omitted from Coase's neoclassical economic justification for the firm. In the mind of the economists, the centralization of power within the firm (i.e. private property) is as naturally occurring as the invisible hand of the market. Going back to the anarchists of old, however, it is instead posited that the hierarchical nature of the firm is just the embodiment of private ownership of property, where to have ownership of property allowed the owner to do what they would in accordance with the law. Thus, the anarchists argued that being the owner of property should be considered as synonymous with 
being, according to Pierre-Joseph Proudhon, a "sovereign lord within the sphere of property, absolute king throughout his own domain."316

This is what led Proudhon to declare that 'Property is Despotism!', which was often accompanied with the proclamation that 'Property is Theft!' being that surplus value generated by labour was taken by those who did not partake in the labour, but simply owned the property (which had a direct influence on Marx's analysis). ${ }^{317}$ Put simply, in a capitalist society where one has to sell their labour to survive, the owners of property are able to rule over and steal from those who do not have property being that the property-less are dependent on the wages of property owners. ${ }^{318}$ This seemingly transgresses against the Friedman notion that 'Political freedom means the absence of coercion of a man by his fellow men'. ${ }^{319}$ In fact, both Proudhon and Marx compared the notion of having to sell one's labour for a wage to that of slavery (which has been popularized over the past several centuries as 'wage slavery').

Being that the institution of private property allows property-holders to so-glaringly trespass upon the rights of others, however, it is dependent on an authority to not only coerce the individuals into accepting their fate, but to protect the property owners from those who are being ruled and stolen from. ${ }^{320}$ In this, the anarchists realized long-ago that this authority came in the form of the state and political authority; per Errico Maletesta, "a special class...provided with the necessary means of repression, exists to legalize and protect the owning class from the

\footnotetext{
316 Pierre-Joseph Proudhon, General Idea of the Revolution (New York: Dover Publications, 1970). 281

317 Pierre-Joseph Proudhon, What is Property? (New York: Dover Publications, 1970); Karl Marx, "On Proudhon" in Marx Engels Selected Works, Volume 2 (Der Social-Demokrat, Nos. 16, 17, and 18, 1865). Available at https://www.marxists.org/archive/marx/works/1865/letters/65_01_24.html.

318 Proudhon, What is Property?, 41

${ }^{319}$ Friedman, Capitalism and Freedom, 15

320 Proudhon, General Idea of the Revolution, 286
} 
demands of the workers." ${ }^{\prime 21}$ In accordance with this, Kropotkin stated that "the rich perfectly well know that if the machinery of the State ceased to protect them, their power over the labouring classes would be gone immediately." ${ }^{322}$ In fact, even Adam Smith stated

The inequality of fortune...introduces among men a degree of authority and subordination...it thereby introduces some degree of that civil government which is indispensably necessary for its own preservation...to maintain and secure that authority and subordination...Civil government, so far as it is instituted for the security of property, is in reality instituted for the defence of the rich against the poor, or of those who have some property against those who have none at all. ${ }^{323}$

Thus, the capitalist market that holds private property as one of its most sacred institutions is entirely dependent on the political authority of the state apparatus to protect this institution, and thus capitalism is dependent on the state; in the words of Kropotkin (and within the same line of thinking as Polanyi), "the State ... and Capitalism are facts and conceptions which we cannot separate from each other. In the course of history these institutions have developed, supporting and reinforcing each other...They are connected with each other -- not as mere accidental co-incidences. They are linked together by the links of cause and effect."1324 Therefore, it should be safe to say that any conception of 'anarcho-capitalism' is also oxymoronic - or perhaps just ahistorical and non-sensical. Capitalism is dependent on private property; private property is dependent on the protection of the state; anarchism is the drive towards the abolition of the state. Thus, anarchism and capitalism are simply antithetical, as the anarchist abhors private property and the capitalist fetishizes it.

\footnotetext{
${ }^{321}$ Errico Malatesta, “An Anarchist Program (1920)" in Anarchism: A Documentary History of Libertarian Ideas, ed. Robert Graham (Montreal: Black Rose Books, 2005). 396

322 Peter Kropotkin, Evolution and Environment (Quebec: Black Rose Books, 1995). 98

${ }^{323}$ Adam Smith, An Inquiry into the Nature and Causes of The Wealth of Nations, Book 5 (Bantam Classics, 1776). 412-3; Anarchist Writers, Why Are Anarchists Against the State?, available at http://anarchism.pageabode.com/afaq/secb2.html.

324 Kropotkin, Evolution and Environment, p. 94
} 
Relating this all back to blockchain, then; it would thus seem that the revolutionary implications of Aragon are somewhat easy to identify when they state "We must strive to create systems in which a large number of diverse stakeholders have a say, in order for common goods to be responsibly governed by their communities." ${ }^{232}$ This notion would seemingly go against the notion of private property and the hierarchical autocracy of the firm, and indeed, it is not so different from when Kropotkin declared "In common with all socialists, the anarchists hold that the private ownership of land, capital, and machinery has had its time; that it is condemned to disappear; and that all requisites for production must, and will, become the common property of society, and be managed in common by the producers of wealth." 326

This notion of the 'producers of wealth' can (and should) be directly correlated to the oftrepeated phrase from the previous sections regarding 'the creators of value'. In lieu of this, many in the blockchain world are starting to hypothesize the true implications regarding the developments of smart contracts, dApps, and DAOs, particularly with how it pertains to the impact the technology can have on the existing economic systems; an organization that is governed by the users that make it up - with all of the value that is being created on that network distributed proportionally to those who are creating it - represents an incredible challenge to the primary tenet of capitalism in the institution of private property: one that facilitates the creation of a sovereign lord ruling over and stealing from those who are creating the value. Rather, than this, we can see what is sometimes referred to as 'platform cooperativism'. ${ }^{327}$

\footnotetext{
325 Aragon, The Aragon Manifesto

${ }^{326}$ Peter Kropotkin, Kropotkin's Revolutionary Pamphlets, edited by Roger N. Baldwin (New York: Dover Publications, 1970). 46

${ }^{327}$ De Filippi, "What Blockchain Means for the Sharing Economy" in Harvard Business Review (15 March, 2017).
} 
The underlying logic of this notion is that the value that is created on these networks is not centralized to the owners and proprietors of the networks, but is distributed amongst those who have contributed to the creation of the value (via the distribution of tokens). ${ }^{328}$ Further, with organizations such as Aragon and Consensys developing the mechanisms to allow for nonhierarchical distribution of network governance, the management of these networks can be done by those who are actually participating in it.

With that being said, however, there are still those that prefer to view blockchain developments within a capitalist purview; Davidson et al., for instance, have analyzed blockchain and argued it to be seen as an institutional innovation, capable of perhaps more efficiently managing the economic activities of individuals. ${ }^{329}$ In this view, blockchain should be considered as an evolutionary development in the institutions of market capitalism, noting the margin upon which blockchain can compete with markets, hierarchies, and relational contracting. ${ }^{330}$ Elsewhere, these authors have suggested that the blockchain could be seen as a technology capable of 'making economies' - though they prefer to think along that Hayekian lines of blockchains being a 'catallaxy' contributing to "the realization of a number of individual objectives which no one knows in their totality." 331 They further cite from Hayek that a catallaxy can "use the knowledge of all participants, and the objectives it serves are the particular objectives of all its participants in all their diversity and polarity," compared to an economy where all members must serve the uniform hierarchy of the firm's objectives in all of their actions. $^{332}$

\footnotetext{
328 Ibid.

${ }^{329}$ Davidson et al., Blockchain and the economic institutions of capitalism.

330 lbid.

${ }^{331}$ Davidson et al., Economics of Blockchain, 11-12

332 Ibid., 1
} 
While there is perhaps merit in the analogy between developing blockchain technology and that of the Hayekian cattalaxy, others have - more aptly in this author's mind - chosen to disregard these hypotheses developed within the dominant economic system's epistemology, instead focusing on a conceptualization centralized around the highlighted notions of Aragon (and in turn Kropotkin) regarding common goods to be responsibly governed by their communities. In this, one of the more common associations for DAOs is with a notion regarding the production and governance of the commons. ${ }^{333}$

Indeed, under the recognition that the discussed networks predominantly exist in the 'information economy' - which connotes an economy mostly associated with the production of knowledge, communication, and information ${ }^{334}$ - it has been argued by Rigi and Prey that information should not be considered as a commodity susceptible to private ownership being that the value of commodities has been traditionally associated with scarcity, whereas information operates under the logic of abundance. ${ }^{335}$ Thus, being that the produced information is still inherently 'valuable' to people (based on their participation within the networks), the argument goes that the production of information should fall under the purview of the 'commons.' 336

The term most often associated with these digital conceptualizations is Commons-Based Peer Production (CBPP), which was coined by Yochai Benkler and refers to socio-economic production under a cooperative model without hierarchy and where resources are shared. ${ }^{337}$ The open-source phenomenon is a typical example of this, which includes operating systems such as

\footnotetext{
${ }^{333}$ Alex Pazaitis, Primavera De Filippi, Vasilis Kostakis, "Blockchain and value systems in the sharing economy: The illustrative case of Backfeed" in Technological Forecasting \& Social Change $(125,2017) .108$

${ }^{334}$ Cited from Pazaitus, Blockchain and Value Systems in the Sharing Economy, 108

335 Ibid.

${ }^{336}$ Ibid.

${ }^{337}$ Yochai Benkler, The Wealth of Networks: How Social Production Transforms Markets and Freedom (New Haven: Yale University Press, 2006).
} 
Linux/GNU; software such as Free/Libre Open Source Software (FLOSS); Wiki's such as Wikipedia; and even the browser Firefox. ${ }^{338}$ In this, CBPP and the organizations that exemplify it could perhaps be seen as early representations of a viable alternative to traditional hierarchical and proprietor-led digital organizations that have a tendency to make the user the 'product'.

Vasilis Kostakis and Michael Bauwens have referred to these hierarchical and proprietorled organizations as 'netarchical capitalism', describing it as 'centralized control of a distributed infrastructure with an orientation toward that accumulation of capital'. ${ }^{339}$ Facebook should be seen as a predominant example of this, but netarchical capitalism essentially describes proprietary platforms held under central control that allow users to share information while having no control over the design or the protocol; according to the authors, "while sharers directly create or share use value, the monetized exchange value is realized by the owners of capital." ${ }^{\prime 40}$ They further go on to describe organizations such as Facebook as neo-feudal being that they engage in severe forms of hyper-exploitation (where the value that is created on the network is almost exclusively realized by the owners of the network). ${ }^{341}$

Though the comparison to feudalism is certainly imperfect, it goes on to say that digital ownership is replaced by access, where an organization like Facebook simply allows a user to have an identity on their network which eliminates self-sovereignty by creating one-sided licensing agreements in the digital sphere. ${ }^{342}$ The analysis can be summed up by saying that users effectively work - toil the fields - for Facebook being that users are the only thing that

\footnotetext{
${ }^{338}$ David Rozas, Antonio Tenorio-Fornes, Silvia Diaz-Molina and Samer Hassan, "When Ostrom Meets Blockchain: Exploring the Potentials of Blockchain for Commons Governance" in SSRN (30 July 2018). 2

${ }^{339}$ Vasilis Kostakis \& Michael Bauwens, Network Society and Future Scenarios for a Collaborative Economy (New York: Palgrave Macmillan, 2014). Chapter 4

340 Ibid.

${ }^{341}$ Ibid.

342 Ibid.
} 
make Facebook worth the hundreds of billions of dollars that it is worth. ${ }^{343}$ However, none of this value that is created is distributed to those who create the value, which is what makes the users the 'product' of the neo-feudal lords - Mark Zuckerberg, for instance, would be the sovereign lord and 'the absolute king through the Facebook domain' in this example.

Counter to this stands the potentials of blockchain-powered DAOs, many of which operate under the full recognition that the value created on the network should be distributed to those who have created the value rather than to those who own the property. In this, the network is a shared resource co-governed by the community, which operates under the rules and norms determined by the users - what David Bollier defines as the commons. ${ }^{344}$ Bollier further posits that decentralized systems under the commons paradigm can help societies transition to new systems of provisioning and democratic governance and away from the deep pathologies of what he refers to as neoliberal capitalism. ${ }^{345}$

Michael Hardt has also recognized the value in reconceptualizing the very notion of value within digital networks, arguing that neoliberal strategies have allowed for the privatization of the digital commons via copyrights and patents. ${ }^{346}$ Hardt contests the logic that allows for this privatization, however, being that the logic of scarcity simply does not hold in this domain there is no 'tragedy of the commons' in the digital realm per se, which means that there is no need for private ownership or public administration in the digital realm because there is no concern that resources shared by individuals acting within their own self-interest (under the

\footnotetext{
${ }^{343}$ Christian Fuchs, "Labor in Informational Capitalism and on the Internet" in The Information Society: The International Journal (26:3, 2010).

344 David Bollier, "Global Citizenship: Plausible Fears and Necessary Dreams" in Great Transition Initiative (April 2014).

345 Ibid.

${ }^{346}$ Michael Hardt, "The Common in Communism" in Rethinking Marxism (22:3, 2010). 350
} 
assumption that individuals will attempt to maximize their own self-benefit) will result in the depletion of common resources. ${ }^{347}$

Still though, the vast amount of digital networks are privately owned and generate immense value solely for the proprietors, even though it is the users of the networks that are producing the value. Hardt and Toni Negri have referred to this digital value creation as immaterial and biopolitical production: the 'production of ideas, information, images, knowledges, code, languages, social relations, affects, and the like.' ${ }^{348}$ Further, they have argued that this form of production has surpassed industry in regard to the hegemonic position that industry once held over sectors of the economy and social relations. ${ }^{349}$ While this argument has been contended (perhaps most vehemently by Marxists), it is without doubt that value creation over digital networks has taken an incredibly prominent role world-wide in regards to social relations and economic production. While this surplus value generation over digital networks has traditionally been privately owned, blockchain and DAOs serve as a direct challenge to this unjustified reality, allowing for collective ownership of these forms of production via commonsbased democratic-governing and provisioning rather than that of private ownership and 'netarchical capitalism' as it has developed over the past several decades.

It is for these reasons that I would therefore assert that participation within the developing networks should be considered as something entirely different than what can be perceived via the dominant epistemological ideology - one that relegates individuals into serving the sovereign lord within the sphere of property. Rather than this, it should indeed be recognized that these networks represent something far more synonymous with the 'communities of affinity' that are

\footnotetext{
347 Ibid., 349

348 Ibid., 348-49

349 Ibid.
} 
emblematic of entirely new understandings of socio-political and economic relationships - as was alluded to in the previous chapter. Regarding these relationships, then; to enter into the idealized DAO would be to enter into the collective governance of the commons 'where we are equally serving and served, rather than just serving. ${ }^{, 350}$ Therefore, participants on the networks are not competing with one another under the watchful-eye of some sort of mightiest force, nor are they simply toiling the fields of Facebook. Rather, they are a member of a collective community directly and actively involved in its evolution, representing that which makes the network come to be whilst simultaneously being represented by its realization.

In terms of a metaphor, Dora Kostakoupoulou has recognized an inspiring parallel regarding the collective governance of these digital communities, and she indeed argues that blockchain technology can help to create a virtual realization of Hannah Arendt's public realm. ${ }^{351}$ In this, Arendt realized that a true public only exists in relation to the governing of the commons, and that the public only comes together in the act of politics to deliberate and decide on how best to manage that which belongs to the domain of humanity - what Hardt chalks up to biopolitical production, Arendt more poetically refers to as "the human artifact, the fabrication of human hands." ${ }^{352}$ Arendt further states that "the term 'public' signifies the world itself, in so far as it is common to all of us and distinguished from our privately owned place in it." ${ }^{353}$ In this light, to enter into a decentralized digital network would be to enter into the common world, and to enter into the common world would thus be to enter into the public - the likes of which would be synonymous with entering into the politics of governing that which belongs to us all.

\footnotetext{
${ }^{350}$ Aragon, The Aragon Manifestio.

${ }^{351}$ Dora Kostakapoulou, "Cloud Agoras: When Blockchain Meets Arendt's Virtual Public" in Debating Transformations of National Citizenship, ed. Rainer Baubock (Switzerland: Springer Open, 2018): 337-341.

${ }^{352}$ Arendt, The Human Condition, 52

353 Ibid.
} 


\section{Concluding Analysis}

To live together in the world means essentially that a world of things is between those who have it in common, as a table is located between those who sit around it; the world, like every inbetween, relates and separates men at the same time.

The public realm, as the common world, gathers us together yet prevents our falling over each other, so to speak. What makes mass society so difficult to bear is not the number of people involved, or at least not primarily, but the fact that the world between them has lost its power to gather them together, to relate and to separate them.

The weirdness of this situation resembles a spiritualistic séance where a number of people gathered around a table might suddenly, through some magic trick, see the table vanish from their midst, so that two persons sitting opposite each other were no longer separated but also would be entirely unrelated to each other by anything tangible.

\section{- Hannah Arendt, The Human Condition.}

To relate all of the concepts developed through each section together, I would strongly posit that what we are discussing - and what we should be presenting - is something far different than that of 'free market arbitration paving the way for humanity to reclaim its personal sovereignty'. To once again critique this notion, conceptualizing the free market acting as the arbiter - if one is to analyze this within the dominant economic epistemology which would be the logical thing to do - assumes private property owners competing with one another in what we refer to as society; per Hayek, "The liberal argument is in favour of making the best possible use of the forces of competition as a means of coordinating human efforts... It is based on the conviction that where effective competition can be created, it is a better way of guiding individual efforts than any other." 354 However, Hayek also admits that "

The functioning of competition not only requires adequate organisation of certain institutions like money, markets, and channels of information - some of which can never be adequately provided by private enterprise - but it depends above all on the existence of an appropriate legal system, a legal system designed both to preserve competition and to make it operate as beneficially as possible. ${ }^{355}$

\footnotetext{
354 Hayek, Road to Serfdom, 37

355 Ibid
} 
This legal system is effectively the state, and while some seem to be making the case that blockchain can essentially replace the state in the provision of its associated institutions, the overarching question that needs to be asked is if you are looking to liberate individuals from the coercive power of the state, then why are you seeking to uphold its most problematic institutions. And further, when you uphold these institutions, what interests will take the place of the state when we are effectively replicating institutions that - as recognized by capitalists and anarchists alike - Are entirely dependent on some sort of state-like apparatus to operate and the therefore necessitate its creation.

In this, it would seem that under the ideology of free market arbitration paving the way for individual sovereignty via the blockchain, all that is implied is that the self-executing nature of contracts in DLT will come to serve as the mightiest force rather than that of the militarized institutions of the state; the social contract would become automated, so to say, in a sort of techno-leviathan. While the critiques associated with this line of thinking (presented briefly in the first chapter) are certainly worth revisiting and expanding upon, they can be summed up with a quote from De Filippi and Wright: "Those working to liberate individuals from the whims of governments and corporations could wind up surrendering themselves (and others) to the whims of a much more powerful entity: autonomous code." 356

The future that blockchain can create is certainly uncertain, and it is important to recognize that the fight for utopia could unwittingly pull us into some form of dystopia if we are not excessively careful regarding how we develop the systems and networks to which people may pledge themselves. In this, it is incredibly important to conceptualize these networks within

${ }^{356}$ De Filippi \& Wright, Blockchain and the Law, 207 
a proper framework, and the question I pose is why would we conceive of this revolutionary technology through the lens of the dominant economic system that inevitably births the political system of which we are supposedly trying to transcend? Further, what are we actually transcending if all we are doing is further committing ourselves in a religious-like fervor to the same erroneous and fetid ideology that has been around for hundreds of years and effectively has not worked; how do you break free by locking yourself into the same system in which you should be trying to escape?

This is why I argue that we should not be discussing blockchain technology within the pretense of free market arbitration. Rather, blockchain is a technology that can facilitate something entirely new and incredibly exciting - but not if it is used to prop up the same institutions that have been plaguing society for centuries; Noam Chomsky plots this risk succinctly:

Anarcho-capitalism, in my opinion, is a doctrinal system which, if ever implemented, would lead to forms of tyranny and oppression that have few counterparts in human history. There isn't the slightest possibility that its (in my view, horrendous) ideas would be implemented, because they would quickly destroy any society that made this colossal error. The idea of 'free contract' between the potentate and his starving subject is a sick joke. ${ }^{357}$

With that being said, however, I am also in accordance with Chomsky when he continues to say that "I should add, however, that I find myself in substantial agreement with people who consider themselves anarcho-capitalists on a whole range of issues...though I do not think they see the consequences of the doctrines they espouse, or their profound moral failings." In this, I would argue that the unscrupulous dedication to the ideology of a free market as the provider of

\footnotetext{
${ }^{357}$ Noam Chomsky, “On Anarchism: Noam Chomsky Interviewed by Tom Lane” in ZNet (23 December 1996), available at https://chomsky.info/19961223/.
} 
freedom is incredibly misguided and symptomatic of what Mark Fischer refers to as 'capitalist realism': the inability to even fathom an alternative to the dominant ideological doctrine because the underlying beliefs have become so embedded that they have effectively become existential truisms. ${ }^{358}$

These beliefs are not truths, however, and to recognize this is to liberate oneself by allowing them to conceptualize actual alternatives to the dominant paradigm which systemically and inevitably creates the coercive political systems that we are trying to transcend in the first place. In this, it is also essential that we recognize blockchain as a tool than can both dismantle the dominant institutions or strengthen them - and we should be fighting to dismantle them if we want to create economic and political systems that are truly different from those that we view as coercive. Further, this fight is also dependent on conceptualizing the future outside of the dominant paradigm and to recognize that blockchain can enable an alternative as long as it is always recognized as an alternative; it is essential that we view this as building a new world rather than recreating the old one.

In order to conceptualize the new world that blockchain can build, then, I would like to once again present the question I posed at the oust of the first chapter: why would we create nations in cyberspace? While this query was meant to more-so serve as a symbolic representation of this essay's broader motif, I would now present it in a more indicative manner to suggest that our imaginations for a new world should not find themselves rooted in the dying gardens of which we used to depend. Rather, they should be cast onto the breeze and allowed to fertilize in whatever soil that will host them. And when the seeds grow and upon the fruition of

${ }^{358}$ Mark Fischer, Capitalist Realism: Is There No Alternative? (United Kingdom: Zero Books, 2009). 
the new garden that comes to be, we must not engage in the same practices that resulted in the death of the one prior; lest we be damned to toiling the same withering fields until the gardens no longer grow.

As has been argued throughout, the nation-state as we know it exists predominantly to protect the private property of self-interested individuals as they compete with one another in what we refer to as a society - though perhaps the term society is misleading in this respect. To once again quote a Conservative Prime Minister of Britain, Margaret Thatcher, "There's no such thing as society. There are individual men and women and there are families." 359 While perhaps it is now obvious that this ought not be read as an endorsement of the politics, it can still be considered as representative of the nation in a liberal democracy. Be that as it may, the preeminent means by which the state facilitates these individualistic values is through its defense and management of the created institutions that allow for a market economy to survive. Further, the true essence of this system is the worship of the free market as the natural triumph of human evolution, as represented by Thatcher's further propagations stating 'there is no alternative' meaning there is nothing better than a society-less group of individuals fighting and competing against one another under the forceful guard of the state and its market.

Historically though, few alternatives have been allowed to develop because individuals have been entirely dependent on this same forceful guard of the state to provide for them and manage their 'true name' which ensures them 'the right to have rights'. To depend on this forceful guard, however, has the effect of relegating the citizens of the nation as subservient to whatever political and economic systems are present in the state in which these rights have been

359 Margaret Thatcher, "A Life in Quotes" in The Guardian (8 April 2013). 
issued. While I have argued that the most contemporary developments regarding self-sovereign identity cannot provide - nor should they provide - the individual the latter declination regarding the right to rights (remembering the Techno-Leviathan), I would say again at this point that there is an incredible potential for emancipation to be found in the liberation of the true name from the jurisprudence of the nation - which is exactly what is possible with a selfsovereign digital identity.

This liberation comes in the form of not necessarily releasing the individual from their subservience to the political and economic systems of which they are relegated, but allowing for them an opportunity to move beyond, seek out, and develop those alternatives which have never really been allowed to flourish; with their true name in hand, the individual might still be bound to the social contract in which they exist, but their avatar is free to roam and build whatever world they want.

I would put it bluntly at this point, however, and state that this avatar has an inherent responsibility to not recreate the chains from which it has escaped; which is to say that it has a responsibility to create the society that the liberal state refutes - one that encourages collectiveness and collaboration as opposed to individualism and competition. Never before has there been such an opportunity to tap into the collective will of not just large groups of people, but humanity in itself. To squander it away by recreating the same institutions of which we have been subservient to for centuries and which divide us as a means of maintaining its own hegemony - not to mention leaving us at the brink of destruction as a species - would be to surrender what could perhaps be considered as the final hope for humanity.

Rather than surrender, however, there is the opportunity to resist. In this, Hardt and Negri - among notable others - have been arguing for decades that the networks of biopolitcal 
production enabled by the internet are ripe for true democratization, which is to say that network organization through the internet is in no need of a centralized and unified command structure (which is becoming more and more apparent with the development of DAOs). Though these arguments long predate the release of blockchain, they have further recognized that the communities that make up the network has no center, only an "irreducible plurality of notes in communication with each other. ${ }^{״ 60}$ In their quest for emancipation, they thus assert that the full democratization of these collections of networks (under the tenets of biopolitical production) ought to be a primary battleground for those seeking alternatives to the dominant paradigm what they refer to as the empire. Importantly, however, their conceptualization states that "Democracy takes the form of a subtraction, a flight, an exodus from sovereignty." 361

In regards to this exodus, these authors recognize the sovereign as not only in a perpetual state of violence and war, but also dependent on a relationship between itself and the subjects where the sovereign dominates as the ruler of the ruled. ${ }^{362}$ Accordingly, they state that "The concept of sovereignty dominates the tradition of political philosophy and serves as the foundation of all that is political precisely because it requires that one must always rule and decide. Only the one can be sovereign, the tradition tells us, and there can be no politics without sovereignty." ${ }^{363}$ However, in their conception of the multitude as horizontally democratic network organization, they presciently argue that "the multitude cannot be reduced to a unity and does not submit to the rule of one. The multitude cannot be sovereign." ${ }^{364}$ The important

\footnotetext{
${ }^{360}$ Antonio Hardt and Michael Negri, Multitudes: War and Democracy in the Age of Empire (New York: Penguin Press, 2004). 83

361 Ibid., 341

362 Ibid., 348

363 Ibid., 329

364 Ibid., 330
} 
distinction worthy of highlighting in this is similar to my broader argument; those making up the multitude must not mimic the political philosophies of yesterday - the code cannot rule.

To move along, however; within this conceptualization, exodus refers to the instance where the multitude comes together declaring 'another world is possible!' and then sets upon itself the task of creating it. ${ }^{365}$ Indeed, per the authors, "Not only must the multitude configure its exodus as resistance, it must also transform that resistance into a form of constituent power, creating the social relations and institutions of a new society." 366 The most effective form of resistance for the multitude to take is therefore the creation of alternatives which counter-impose the violence of the sovereign with the peaceful democracy of an exodus. This act will further serve to drain the energy of the empire and will render it redundant, automatically giving more legitimacy to the forms of exodus which are coming to serve as the alternative networks. In sum, independent struggles for democracy interlinked in a distributed network organization will come to displace the empire of domination by rendering it redundant through its provision of a superior alternative.

It is my assertion that this exodus can now be enabled via the provision of SSDI. Be it through Bitnation, Everynym, u-Port, Veres One, Civic, or some other yet-to-be analyzed organization, it is the ability for an individual to self-assert themselves with full control within the developing digital networks that can allow for them the ability to tread a new path. Indeed, to put it another way, by dismantling the reliance on the institutions of the sovereign state to decide whether or not someone 'belongs to humanity' allows for the sovereign individual to actually enter into humanity - the fruits of which we have never really tasted.

\footnotetext{
365 Ibid., 348

366 Ibid.
} 
Under this precipice, then, I would therefore posit that it is most essential to engage in how these networks of self-sovereign identity are to be developed. Upon this recognition, however - and as a bit of a digression meant to foreshadow the closing appeal - I would plant a seed in the reader's mind with the argument that perhaps we should not be conceptualizing the need for SSDI as the need for one of these networks to triumph above the rest to provide for us our liberation (which would seemingly fall under the purview of capitalist competition). Rather, perhaps we should understand the transcendence to SSDI as a collection of all of relevant organizations acting as independent nodes on an unidentified and unattributed global 'network'; one that is not so dissimilar to how the nodes would operate on a decentralized autonomous organization - independently yet effectively in unison. In essence, and in keeping with Hardt and Negri, I am arguing that perhaps it would be most appropriate to identify digital identity as its own sort of institution. Further, we should consider this institution as a globally open-source commons network - where the open-source networks working autonomously within this parameter operate as a collective whole. What it would take to enable this is certainly beyond my individual prognostication (which is somewhat implicitly ironic), but I do consider it very worthy of appropriate recognition and principle in my particular vision. Perhaps a metaphor would be what is desired from Evernym and the Sovrin Foundation regarding the stewards of identity.

Before I engage fully with this foreshadow, however, we must continue to build up from SSDI allowing individuals to enter humanity in the exodus. In this, while I would certainly not make the case that to enter a social network would be to enter humanity, there is something to the notion of participation on networks such as Steemit, Sapien, Choon, Consensys, and Aragon that can be conceived as 'liberating'; are we not bypassing the 'sovereign lord within the domain 
of property' and instead engaging in a democratic collective governance of the commons? As a hypothetical, imagine all were to move away from the dictatorial ruling of Facebook and towards a Democratized Autonomous Platform such as Sapien, where users are not only able to participate in DAP voting to help influence the company roadmap and organization, but are effectively paid just for the value that they create. Would this not seemingly be representative of 'the peaceful democracy of an exodus'? Does the same notion not apply to the other organizations highlighted in the second chapter? Finally, could it not be applied to other networked organizations operating under the logic of netarchical capitalism - Instagram and Youtube could be considered prime candidates, for instance, but even corporations like Uber are vulnerable to a decentralized alternative.

And before we douse this unfettered idealism with a dose of pragmatism, extrapolating on this ideal as a notion provides for us a blank canvas upon which we can sketch out an alternative socio-political paradigm. Under the purview of biopolitical production, these sorts of networks are premised on producing ideas, information, images, knowledges, and social relations; the production of which holds an inherent value that is distributed to those of who are involved in its creation. And while this in itself could enable entirely new avenues for selfgenerated income - perhaps as a counter to the supposed need for universal basic income - it would be a true shame to limit our imagination to the redistribution of value on social networks.

Indeed, while an SSDI would be able to enter into a decentralized social media network, they would also be able to enter into other potential networks that are truly awe-inspiring. Harking back to our example of identity as an institution and operating under the premise that something like the epitomized Sovrin Foundation were to emerge, individuals would be able to enter into this network and directly engage with its governance as a collective whole - even if 
they are only involved in one of the networks that makes up the broader network of the institution of identity. A user can engage solely in the governance of Bitnation, for instance, but Bitnation would be involved in the governance of identity as a whole; the individual's contribution would thus reverberate throughout the entirety - democracy interlinked in a distributed network organization.

And while there is admittedly a certain socio-political naivety in these sorts of idealistic proclamations, it is not hard to conceptualize alternative institutional networks operating under the same sort framework - such as ones dealing with climate change, scientific progress, local and global infrastructure, sustainable and ethical manufacturing, renewable energy, terrorism, direct democracy, agriculture and food production, ocean clean-up, the UN's sustainable development goals, indigenous reconciliation, and so on and so forth; the examples are effectively infinite. And though some will inevitably choose to instead chalk this up as starryeyed idealism, I would quote the creator of the World Wide Web, Tim Berners-Lee, in response, saying: "It's not naive to think we can have that, but it is naive to think we can just sit back and get it." 367

Many of the organizations highlighted throughout the duration of this essay are emblematic of 'going out to get it' as they engage in incredible forms of praxis. Further, there is one final organization worthy of recognition in this context, and that is Democracy Earth. While it would not be practical at this point to go into full detail regarding the technology that they are developing, suffice it to say that they have some of the more pragmatic and rigorous conceptualizations and technological capabilities in the blockchain world. Further, their entire

\footnotetext{
${ }^{367}$ Democracy Earth (White Paper), Democracy Earth, The Social Smart Contract (Creative Commons AttributionNon-Commercial ShareAlike 4.0 International License, 25 January 2018). 3
} 
organization is premised around the statement "We seek nothing less than true democratic governance for the Internet age.” ${ }^{368}$ The technology they are developing thus has a primary focus on granting 'participation rights to every human with decision-making as its main function' and the organization fully recognizes self-sovereign identity as instrumental in this task. ${ }^{369}$ Finally, in regards to their political philosophy, they state that "there's no reason stopping mankind from building a borderless commons that can help shape the next evolutionary leap for democratic governance at any scale." 370

I would expand on their proclamation to state that this should be nothing less than our collective desire, and while the democratic governing services being developed by Bitnation are truly remarkable - and likely necessary in the networks to come - to conceptualize what can be built within the thinking of the cypherpunks regarding free market arbitration and competition would be to further envelop societies within the same dominant paradigm of which we should be looking to transcend. Rather than this, we should be conceptualizing what blockchain can enable within a paradigm parallel to that of the democratic exodus or entrance into the public; lest it be appropriated and come to serve as a tool for the dominant regime. In this, Audre Lorde once symbolically argued that 'The Master's Tools Will Never Dismantle the Master's House. ${ }^{\text {'371 }}$ While I would not wish to appropriate her critique to serve my own agenda - a painful irony to those familiar with her work - I do consider it to be the strongest allegory for how we should be conceptualizing the blockchain future.

\footnotetext{
368 Ibid.

369 Ibid.

370 Ibid., 3-4

${ }^{371}$ Audre Lorde, "The Master's Tools Will Never Dismantle the Master's House," in Sister Outsider: Essays and Speeches (Berkeley, CA: Crossing Press, 2007). 110-114.
} 
Blockchain is an alternative tool and should always be recognized as such. In this, however, a tool is only useful when it is held by a person, and it is only the people that can decide what it is they want to build.

I wish to build the table so that we can collectively enjoy what our gardens come to grow. 


\section{$\underline{\text { References }}$}

Abate, Janet. "True Risks? The Pleasures and Perils of Cyberspace." Science Fiction and Computing: Essays on Interlinked Domains, ed. David Ferro \& Eric Swedin. Mcfarland, 2011

ABI Research. Blockchain-Based Cybersecurity. 2017. Available at https://cdn2.hubspot.net/hubfs/2241249/Blockchain_Based_Cybersecurity_Report.pdf. [accessed 17 March 2019]

Access Now. "National Digital Identity Programmes: What's Next?” May 2018, available at https://www.accessnow.org/cms/assets/uploads/2018/06/Digital-Identity-Paper-201805.pdf [accessed 17 March 2019]

Access Now. "Supreme Court of India rules to restrict world's largest digital identity framework (Aadhaar) - but debate continues." 26 September 2018, available at https://www.accessnow.org/supreme-court-of-india-rules-to-restrict-worlds-largestdigital-identity-framework-aadhaar-but-debate-continues/ [accessed 17 March 2019]

Allen, Christopher. The Path to Self-Sovereign Identity. (Blog). 25 April 2016, available at http://www.lifewithalacrity.com/2016/04/the-path-to-self-soverereignidentity.html\#dfref-1111 [accessed 17 March 2019].

Amnesty International. "For Your Eyes Only?" Amnesty International. 2016, available at https://www.amnesty.org/download/Documents/POL4049852016ENGLISH.PDF [accessed 17 March 2019]

Anarchist Writers, Why Are Anarchists Against the State? Available at http://anarchism.pageabode.com/afaq/secb2.html [accessed 17 March 2019]

Aragon. Aragon Network. Available at https:/github.com/aragon/whitepaper [accessed 17 March 2019]

Aragon. The Aragon Manifestio (8 May 2018), available at https://blog.aragon.org/the-aragonmanifesto-4a21212eac03/ [accessed 17 March 2019]

Aragon. The World's First Digital Jurisdiction. Available at https://aragon.org/network/ [accessed 17 March 2019]

Arendt, Hannah. The Human Condition. Chicago: The University of Chicago Press, 1958

Arendt, Hannah. The Origins of Totalitarianism. Berlin: Schocken Books, 1951

Barlow, John Perry. "A Declaration of the Independence of Cyberspace." Crypto-Anarchy, Cyberstates, and Pirate Utopias, ed. Peter Ludlow. Cambridge: MIT Press, 2001

Baubock, Rainer. “Citizenship in Cloud Cuckoo Land?” Debating Transformations of National 
Citizenship, ed. Rainer Baubock. Switzerland: Springer Open, 2018

Bearak, Max. "Theresa May criticized the term 'citizen of the world.' But half the world identifies that way." The Washington Post (5 October 2016). Available at https://www.theatlantic.com/technology/archive/2018/02/virtual-citizenship-forsale $/ 553733$

Beck, Estee. "The Invisible Digital Identity: Assemblages in Digital Networks." Computers and Compositions 35 (March 2015)

Bender, Jens. eIDAS Regulation: eID - Opportunities and Risks (January 2015), available at https://www.bsi.bund.de/SharedDocs/Downloads/DE/BSI/ElekAusweise/SmartCard_Wo rkshop/Workshop_2015_Bender.pdf? [accessed 17 March 2019]

Beniiche, Abdeljalil. "Ethereum: Decentralized Applications and Autonomous Organizations" in Zeitgeist Labs. 2018, available at http://www.zeitgeistlab.ca/doc/Ethereum_Decentralized_Applications_Autonomous_Org anizations.html [accessed 17 March 2019]

Benkler, Yochai. The Wealth of Networks: How Social Production Transforms Markets and Freedom. New Haven: Yale University Press, 2006

Belgian Privacy Commission. Facebook Tracking Through Social Plug-ins. 27 March 2015, available at https://securehomes.esat.kuleuven.be/ gacar/fb_tracking/fb_pluginsv1.0.pdf [accessed 17 March 2019]

Bitcago Crypto. "How Referum Works." Youtube Video (2 February 2018), available at https://www.youtube.com/watch?v=316VclIRRS8 [accessed 17 March 2019]

Bitcoinist.net. "D2P3 - TNABC 2015 - VITALIK BUTERIN FOUNDER ETHEREUM - Bitcoin 2.0 - Ideas and Applications." Youtube video (26 January 2015), available at https://www.youtube.com/watch?v=Fjhe0MVRHO4 / [accessed 17 March 2019]

Bitnation. Bitnation Governance 2.0. 5 March 2018, available at https:/github.com/BitNation/Pangea-Docs/blob/master/BITNATION\%20Pangea\%20Whitepaper\%202018.pdf [accessed 17 March 2019]

Bitnation. "PRESS RELEASE: Bitnation releases first batch of Vienna Convention compliant diplomatic blockchain passports." Steemit. November 2018, available at https://steemit.com/bitnation/@bitnation/press-release-bitnation-releases-first-batch-ofvienna-convention-compliant-diplomatic-blockchain-passports [accessed 17 March 2019]

Blake, Michael. "Virtual Politics, Real Guns: On Cloud Community, Violence, and Human Rights." Debating Transformations of National Citizenship, ed. Rainer Baubock Switzerland: Springer Open, 2018

Bollier, David. "Global Citizenship: Plausible Fears and Necessary Dreams." Great Transition 
Initiative. April 2014

Botsman, Rachel. Who Can You Trust: How Technology Brought Us Together and Why It Might Drive Us Apart. New York Books: Public Affair Books, 2017

Bryson, Dave, Dave Penn, David C. Goldenberg \& Gloria Serrao. "Blockchain Technology for Government." Mitre Technical Report. 2018, available at https://www.mitre.org/sites/default/files/publications/blockchain-technology-forgovernment-18-1069.pdf [accessed 17 March 2019]

Buterin, Vitalik. "DAOs, DACs, DAs and More: An Incomplete Terminology Guide" in Ethereum Blog. 6 May 2014, available at https://blog.ethereum.org/2014/05/06/daosdacs-das-and-more-an-incomplete-terminology-guide/ [accessed 17 March 2019]

Cadwalldr, Carol. "The great British Brexit robbery: how our democracy was hijacked." The Guardian. 7 May 2017, available at https://www.theguardian.com/technology/2017/may/07/the-great-british-brexit-robberyhijacked-democracy [accessed 17 March 2019]

Caribou Digital. Private-Sector Digital Identity in Emerging Markets. 2016. Farnham, Surrey, United Kingdom, available at http://cariboudigital.net/new/wpcontent/uploads/2016/08/Caribou-Digitial-Omidyar-Network-Private-Sector-DigitalIdentity-In-Emerging-Markets.pdf [accessed 17 March 2019]

Cheng, Allen. “Alibaba vs. The World." Institutional Investor. 25 July 2017, available at https://www.institutionalinvestor.com/article/b1505pjf8xsy75/alibaba-vs-the-world [accessed 17 March 2019]

Choon, Crypto \& Tunes (2019), available at https://choon.co/about [accessed 17 March 2019]

Choon. Choon: The Blockchain's Music Solution, available at https://www.ch oon.co/public/pdf/choon_white_paper.pdf [accessed 17 March 2019]

Chomsky, Noam. "On Anarchism: Noam Chomsky Interviewed by Tom Lane.” ZNet. 23 December 1996, available at https://chomsky.info/19961223/ [accessed 17 March 2019]

Circles. "Fair Money for All: Basic Income on the Blockchain." Wordpress. 16 February 2017, available at https://ourbasicincome.wordpress.com [accessed 17 March 2019]

Civic. Secure Identity Platform. 2019, available at https://www.civic.com/products/secureidentity-platform/ [accessed 17 March 2019]

Civil. Stories that Need Telling (The Civil Media Company, 2019), available at https://civil.co/ [accessed 17 March 2019]

Coase, R. H. "The Nature of the Firm." Wiley Online Library. November 1937 
Colt, Sam. "Tim Cook Has An Open Letter To All Customers That Explains How Apple's Privacy Features Work.” Business Insider. 17 September 2014, available at https://www.businessinsider.com/tim-cook-published-a-letter-on-apple-privacy-policies2014-9 [accessed 17 March 2019]

Consensys. About Us (2019), available at https://consensys.net/about/ [accessed 17 March 2019]

Consensys. "Consensys Monthly Report - October 2018." Medium. 31 October 2018, available at https://media.consensys.net/consensys-monthly-report-october-20187f8bae68842b [accessed 17 March 2019]

ConsenSys Media. "OE LUBIN - Nature of The Firm, v2.0 KEYNOTE from EtherealNY \#Blockchain Conference 2018." Youtube Video (13 May 2018), available at https://www.youtube.com/watch?v=SQbcGhnv4jw [accessed 17 March 2019]

Constine, Josh. "Facebook Acquires Biometric ID Verification Startup Confirm.io." TechCrunch. 2018, available at https://techcrunch.com/2018/01/23/facebook-confirm-io/ [accessed 17 March 2019]

Creemers, Rogier. "China's Social Credit System: An Evolving Practice of Control." SSRN. 9 May 2018, available at http://www.iberchina.org/files/2018/social_credit_china.pdf [ accessed 17 March 2019]

Dangyang, Zhang. "Guangzhou Issues the first Wechat Identification Card, Expected to Launch Nationwide in coming January.” Xinghua. 26 December 2017, available at http://www.xinhuanet.com/politics/201712/26/c_1122165668.htm?fbclid=IwAR1d2XUSRgthN_Wf_rO0vahNPsEFfmyyDggSV H6G28fuzMfeR-dOBYY3Reo [accessed 17 March 2019]

Davidson, Sinclair, Primavera De Filippi \& Jason Potts. "Blockchain and economic institutions of capitalism." Journal of Institutional Economics. 14:4, 2018

Davidson, Sinclair, Primavera De Filippi \& Jason Potts. "Economics of Blockchain.” SSRN, 8 March 2016

De Filippi, Primavera. "Citizenship in the Era of Blockchain-Based Virtual Nations." Debating Transformations of National Citizenship, ed. Rainer Baubock. Switzerland: Springer Open, 2018: 267-277.

De Filippi, Primavera \& Aaron Wright. Blockchain and the Law. Cambridge: Harvard University Press, 2018

De Filippi, Primavera. "What Blockchain Means for the Sharing Economy." Harvard Business Review. 15 March 2017

Digital Identity \& Authentication Coalition of Canada. Building Canada's Digital Identity 
Future. May 2015, available at https://diacc.ca/wp-content/uploads/2015/05/DIACC-

Building-Canadas-Digital-Future-May-6-2015.pdf [accessed 17 March 2019]

Digital Identity \& Authentication Council of Canada. Digital Identity Innovation - Canada's Opportunity to Lead the World. 4 August 2017, available at https://www.ourcommons.ca/Content/Committee/421/FINA/Brief/BR9073373/brexternal/DigitalIDAndAuthenticationCouncilOfCanada-e.pdf [accessed 17 March 2019]

Digital Identity \& Authentication Council of Canada. Membership. 2019, available at https://diacc.ca/members/ [accessed 17 March 2019]

Digital Identity \& Authentication Council of Canada. The Economic Impact of Digital Identity in Canada: Understanding the potential for considerable economic benefits and the cost of inaction. 2018, available at https://diacc.ca/wp-content/uploads/2018/05/EconomicImpact-of-Digital-Identity-DIACC-v2.pdf [accessed 17 March 2019]

Democracy Earth. Democracy Earth, The Social Smart Contract. Creative Commons Attribution-Non-Commercial ShareAlike 4.0 International License, 25 January 2018, available at https://www.dropbox.com/s/sifogl4zimwkkei/Democracy\%20Earth\%20$\% 20$ Social\%20Smart\%20Contract\%20-\%20Paper\%20v0.2.pdf?dl=0. 3 [accessed 17 March 2019]

Digilocker National e-Governance Division. Digilocker User Manual. Available at https://digilocker.gov.in/assets/img/DigiLocker-User-Manual.pdf

Dupont, Quinn \& Bill Maurer. "Ledgers and Law in The Blockchain.” Kings Review. 23 June 2015, available at http://kingsreview.co.uk/articles/ledgers-and-law-in-the-blockchain/ [accessed 17 March 2019]

E-Estonia. E-identity. Available at https:/e-estonia.com/solutions/e-identity/e-residency [accessed 17 March 2019]

E-Estonia. “E-Governance.” Available at https://e-estonia.com/solutions/e-governance/ [accessed 17 March 2019]

E-Estonia. Estonian-Finnish e-Governance without borders. Available at https://e-estonia.com/estonian-finnish-e-governance-without-borders/ [accessed 17 March 2019]

E-Estonia. How do Estonians save annually 820 years of work without much effort? December 2017, available at https:/e-estonia.com/how-save-annually-820-years-of-work/ [accessed 17 March 2019]

E-Estonia. Interoperability Services. Available at https:/e-estonia.com/solutions/interoperability- 
services/ [accessed 17 March 2019]

Economy, Elizabeth. The Third Revolution: Xi Jinping and the New Chinese State. England: Oxford University Press, 3 May 2018

Emmadi, Nitesh \& Harika Narumanchi. "Reinforcing Immutability of Permissioned Blockchains with Keyless Signatures' Infrastructure.” Proceedings of the 18th International Conference on Distributed Computing and Networking 46, 05 January 2017

Ethereum. A Next-Generation Smart Contract and Decentralized Application Platform. 2015, available at https://github.com/ethereum/wiki/wiki/White-Paper [accessed $17 \mathrm{March}$ 2019]

Ethereum. Web 3: A platform for decentralized apps. 2016, available at http://ethdocs.org/en/latest/introduction/web3.html [accessed 17 March 2019]

Ethereum, "What is Ethereum?" Youtube Video (4 June 2014), available at https://www.youtube.com/watch?time_continue $=82 \& \mathrm{v}=\mathrm{Clw}-\mathrm{qf} 1 \mathrm{sUZg}$

European Union European Regional Development Fund. Estonian Blockchain Technology. Available at https://e-estonia.com/wp-content/uploads/faq-a4-v02-blockchain.pdf [accessed 17 March 2019]

Facebook. Cookies \& Other Storage Technologies. 4 April 2018, available at https://www.facebook.com/policy/cookies/ [accessed 17 March 2019]

Facebook. Data Policy. 19 April 2018, available at https://www.facebook.com/full_data_use_policy [accessed 17 March 2019]

Facebook. Facebook Principles. 2019, available at https://www.facebook.com/principles.php [accessed March 2019]

Finland Population Register Centre. Finland's and Estonia's data exchange layers connected to one another on 7 February - the rapid exchange of information between the countries is now possible (Press release). 8 February 2018, available at https://vrk.fi/en/article//asset_publisher/suomen-ja-viron-palveluvaylat-liitetty-yhteen-7-2-tietojen-nopea-jaluotettava-vaihto-maiden-valilla-nytmahdollista?lipi=urn\%3Ali\%3Apage\%3Ad_flagship3_feed\%3BZcq8b6EuT8Wf7N5dlZj 7LQ\%3D\%3D [accessed 17 March 2019].

Fischer, Mark. Capitalist Realism: Is There No Alternative? United Kingdom: Zero Books, 2009

Fear, David. “'Black Mirror': Inside New Season of Chilling Cult U.K. Horror Series.” Rolling Stone. 13 October 2016, available at https://www.rollingstone.com/tv/tv-features/black-mirror-inside-new-season-of-chillingcult-u-k-horror-series-120805/ [accessed 17 March 2019] 
Friedman, Milton. Capitalism and Freedom. Chicago: The University of Chicago Press, 1962

Fuchs, Christian. "Labor in Informational Capitalism and on the Internet." The Information Society: The International Journal 26:3, 2010.

Garrod, JZ. “The Real World of Decentralized Autonomous Society.” TripleC. 14:1, 2016

Gerring, Taylor. "Cut and dry: building a dream." Ethereum Blog. 9 February 2016, available at https://blog.ethereum.org/2016/02/09/cut-and-try-building-a-dream/ [accessed 17 March 2019]

Godwin, William. An Enquiry Concerning Political Justice. London: G. G.J. and J. Robinson, 1793

Government of India. "Benefits of Aadhaar Card." Vikaspedia, 2019: available at http://vikaspedia.in/e- governance/online-citizen-services/government-to-citizen-servicesg2c/all-about-aadhaar/17-benefits-of-aadhaar-card [accessed 17 March 2019]

Government of India. "Digilocker: Your Documents Anytime, Anywhere." Digilocker. 2019, available at https://digilocker.gov.in/ [accessed 17 March 2019]

Graeber, David. Toward and Anthropological Theory of Value. New York: Palgrave Macmillan, 2001

Hardt, Michael, and Antonio Negri. Multitude: War and Democracy in the Age of Empire. New York: The Penguin Press, 2004.

Hardt, Michael. "The Common in Communism.” Rethinking Marxism 22:3, 2010

Hayek, Friedrich. The Road to Serfdom. New York: Routledge Publishing, 1944

Hazari, Gautam. "The Relationship Between Blockchain and Digital Identity." GSMA November 2016, available at https://www.gsma.com/identity/the-relationship-between-blockchainand-digital-identity [accessed 17 March 2019]

Hautala, Laura. "The Snowden effect: Privacy is good for business." CNET. 3 June 2016, Available at https://www.cnet.com/news/the-snowden-effect-privacy-is-good-forbusiness-nsa-data-collection/ [accessed 17 March 2019]

Harvey, David. A Brief History of Neoliberalism. Oxford: Oxford University Press, 2005

Hsieh,Ying-ying \& Jean-Philippe Vergne. "Bitcoin and the Rise of Decentralized Autonomous Organizations.” SSRN. 11 December 2017

Hughes, Eric. “A Cypherpunk’s Manifesto.” Crypto-Anarchy, Cyberstates, and Pirate Utopias, ed. Peter Ludlow. Cambridge: MIT Press, 2001 
Hughes, Neil. "UN Will Use Blockchain and Digital Identities to Combat Child Trafficking." One World Identity. 13 November 2017, available at https://oneworldidentity.com/unwill-use-blockchain-digital-identities-combat-child-trafficking/ [accessed 17 March 2019]

Iamsysteemiamet, "X-Road Introduction (Long version)" Youtube video (10 June 2016), available at https://www.youtube.com/watch?v=b-r6B28qVSY [accessed 17 March 2019]

Iqbal, Mansoor. "WeChat Revenue and Usage Statistics (2019)." Business of Apps. (27 February 2019), available at http://www.businessofapps.com/data/wechat-statistics/\#1 [accessed 17 March 2019]

ID2020. The Alliance Manifesto. 2019, available at https://id2020.org/manifesto/

Identity 2020 Systems. "The Need for Good Digital ID is Universal.” 2019, available at https://id2020.org/digital-identity-1/ [accessed 17 March 2019]

Irrera, Anna. "Accenture, Microsoft team up on blockchain-based digital ID network." Reuters. 19 June 2017, available at https://www.reuters.com/article/us-microsoft-accenturedigitalid/accenture-microsoft-team-up-on-blockchain-based-digital-id-networkidUSKBN19A22B [accessed 17 March 2019]

Janrain. "What are the common types of authentication protocols?" Janrain. 2019, available at https://www.janrain.com/resources/question-and-answers/authentication/what-arecommon-types-authentication-protocols [accessed 17 March 2019]

Juskalian, Russ. "Inside the Jordan refugee camp that runs on blockchain." MIT Technology Review. 12 April 2018, available at https://www.technologyreview.com/s/610806/insidethe-jordan-refugee-camp-that-runs-on-blockchain/ [accessed 17 March 2019]

Kames. "The Basics of Decentralized Identity.” Medium. 26 June 2018, available at https://medium.com/uport/the-basics-of-decentralized-identity-d1ff01f15df1 [accessed 17 March 2019]

Kames. "Why Decentralized Identity Is Essential to Scaling the Ethereum Blockchain." Medium. 20 June 2018, available at https://medium.com/uport/why-decentralizedidentity-is-essential-to-scaling-the-ethereum-blockchain-58f6dc1bd1d1 [accessed 17 Marh 2019]

Kaste, Martin. "Who Are You Really? Activists Fight For Pseudonyms." NPR. 28 September 2011, available at https://www.npr.org/2011/09/28/140879480/who-are-you-reallyactivists-fight-for-pseudonyms [accessed 17 March 2019]

Kivimaki, Petteri. "There is no blockchain technology in the X-Road. Nordic Institute for Interoperability Solutions. 26 April 2018, available at https://www.niis.org/blog/2018/4/26/there-is-no-blockchain-technology-in-the-x-road [accessed 17 March 2019] 
Kohlhaas, Paul. "Zug ID: Exploring the First Publicly Verified Blockchain Identity." Medium. 6 December 2017, available at https://medium.com/uport/zug-id-exploring-the-firstpublicly-verified-blockchain-identity-38bd0ee3702 [accessed 17 March 2019]

Korjus, Kaspar. "Estonian President Kersti Kaljulaid reveals the future direction of eResidency." Medium. 18 December 2018, available at https://medium.com/e-residencyblog/estonian-president-kersti-kaljulaid-reveals-the-future-direction-of-e-residency5b1177dfa78c [accessed 17 March 2019]

Korjus, Kasper. Welcome to the Blockchain Nation. 7 July 2017, available at https://medium.com/e-residency-blog/welcome-to-the-blockchain-nation-5d9b46c06fd4 [accessed 17 March 2019]

Kostakis Vasilis \& Michael Bauwens. Network Society and Future Scenarios for a Collaborative Economy. New York: Palgrave Macmillan, 2014

Kostakapoulou, Dora. “Cloud Agoras: When Blockchain Meets Arendt's Virtual Public.” Debating Transformations of National Citizenship, ed. Rainer Baubock. Switzerland: Springer Open, 2018: 337-341.

Kropotkin, Peter. Evolution and Environment. Quebec: Black Rose Books, 1995

Kropotkin, Peter. Kropotkin's Revolutionary Pamphlets, edited by Roger N. Baldwin New York: Dover Publications, 1970

Kuo, Lily. "China bans $23 \mathrm{~m}$ from buying travel tickets as part of 'social credit' system." The Guardian. 1 March 2019, available at https://www.theguardian.com/world/2019/mar/01/china-bans-23m-discredited-citizensfrom-buying-travel-tickets-social-credit-system [accessed 17 March 2019].

Le Bras, Tom. "Online Overload - It's Worse Than You Think.” Dashlane. 21 July 2015, available at https://blog.dashlane.com/infographic-online-overload-its-worse-than-youthought/ [accessed 17 March 2019]

Levine, Barry. "What's the Most Popular Social Login on B2B Sites?" CMS Wire. 15 July 2014, available at https://www.cmswire.com/cms/customer-experience/whats-the-mostpopular-social-login-on-b2b-sites-025874.php [accessed 17 March 2019]

Lorde, Audre. "The Master's Tools Will Never Dismantle the Master's House." Sister Outsider: Essays and Speeches. Berkeley, CA: Crossing Press, 2007. 110-114.

Ludlow, Peter. Crypto-Anarchy, Cyberstates, and Pirate Utopias. Cambridge: MIT Press, 2001

Lundkvist, Christian, Rouven Heck, Joel Torstensson, Zac Mitton \& Michael Sena. uPort: A 
Platform for Self-Sovereign Identity. 20 October 2016, available at http://blockchainlab.com/pdf/uPort_whitepaper_DRAFT20161020.pdf [accessed 17 March 2019].

Macaskill, Ewen \& Gabriel Dance. "NSA Files Decoded: What the Revelations Mean For You." The Guardian. 1 November 2013, available at https://www.theguardian.com/world/interactive/2013/nov/01/snowden-nsa-filessurveillance-revelations-decoded\#section/1 [accessed 17 March 2019]

Malatesta, Errico. “An Anarchist Program (1920)." Anarchism: A Documentary History of Libertarian Ideas, ed. Robert Graham. Montreal: Black Rose Books, 2005.

Mastercard Press Release. Mastercard, Microsoft Join Forces to Advance Digital Identity Initiatives. 3 December 2018, available at https://newsroom.mastercard.com/pressreleases/mastercard-microsoft-join-forces-to-advance-digital-identityinnovations/\#_prclt=IarCgTyK [accessed 17 March 2019]

Marx, Karl. "On Proudhon" in Marx Engels Selected Works, Volume 2. Der Social-Demokrat, Nos. 16, 17, and 18, 1865

May, Timothy. "Crypto Anarchy and Virtual Communities." Crypto-Anarchy, Cyberstates, and Pirate Utopias, ed. Peter Ludlow. Cambridge: MIT Press, 2001

Metamask. Brings Ethereum to your browser. Available at https://metamask.io/ [accessed 17 March 2019]

Nabi, Atif Ghulam. "Comparative Study on Identity Management Methods Using Blockchain." University of Zurich. 29, September 2017, available at https://files.ifi.uzh.ch/CSG/staff/Rafati/ID\%20Management\%20using\%20BC-AtifVA.pdf [accessed 17 March 2019]

Nakamoto, Satoshi. Bitcoin: A Peer-to-Peer Electronic Cash System. Available at https://bitcoin.org/bitcoin.pdf

Nakamoto, Satoshi. Email from Satoshi Nakamoto on November 11 $1^{\text {th }}$ 2009, available at http://satoshi.nakamotoinstitute.org/emails/cryptography/12

Open Law. Building the Future of Law, One Legal Agreement and Contract at a Time (Aaron Wright, David Roon, and ConsenSys AG, 2019), available at https://www.openlaw.io/ [accessed 17 March 2019]

Orgad, Liav. "Cloud Communities: The Dawn of Global Citizenship?" in Debating Transformations of National Citizenship, ed. Rainer Baubock. Switzerland: Springer Open, 2018

Owen, Malcolm. "Apple wants iPhone to be proof of identity and replace passports." Apple Insider. 9 August 2018, available at https://appleinsider.com/articles/18/08/09/apple- 
wants-iphone-to-be-proof-of-identity-and-replace-passports [accessed 17 March 2019]

Parker, Luke. "Bitnation starts offering blockchain public notary service to Estonian e-

Residents." Brave New Coin. 30 November 2015, available at

https://bravenewcoin.com/insights/bitnation-starts-offering-blockchain-public-notaryservice-to-estonian-e-residents [accessed 17 March 2019]

Pazaitis, Alex, Primavera De Filippi, and Vasilis Kostakis. "Blockchain and value systems in the sharing economy: The illustrative case of Backfeed." Technological Forecasting \& Social Change 125, 2017

Polanyi, Karl. The Great Transformation: The Political and Economic Origins of Our Time. Boston: Beacon Press Books, 1944

Proudhon, Pierre-Joseph. General Idea of the Revolution. New York: Dover Publications, 1970

Proudhon, Pierre-Joseph. What is Property? New York: Dover Publications, 1970

Pybus, Robert. “The Uncanny Mind that Built Ethereum.” Wired. 13 June 2016, available at https://www.wired.com/2016/06/the-uncanny-mind-that-built-ethereum/ [accessed 17 March 2019]

Refereum. "1 Million Refereum Users | 2018 Year End Review” in Medium. 21, December 2018, available at https://medium.com/refereum/1-million-refereum-users-2018-yearend-review-21397ad9be19 [accessed 17 March 2019]

Refereum. Earn rewards for playing and sharing games (2019), available at https://refereum.com/games [accessed 17 March 2019]

Refereum. Refereum: Referrals in the blockchain (25 January 2018), available at https://old.refereum.com/info/whitepaper [accessed 17 March 2019]

Reijers, Wessel, Fiachra O’Brolchain, and Paul Haynes. “Governance in Blockchain Technologies \& Social Contract Theories.” Ledger.org 1 (2016)

Republic of Estonia. The New Digital Nation. Available at https://e-resident.gov.ee/ [accessed 17 March 2019]

Rhodes, Yorke III. "What does identity mean in today's physical and digital world." Microsoft Azure. 31 May 2016, available at https://azure.microsoft.com/en-us/blog/what-doesidentity-mean-in-today-s-physical-and-digital-world/ [accessed 17 March 2019]

Rozas, David, Antonio Tenorio-Fornes, Silvia Diaz-Molina and Samer Hassan. "When Ostrom Meets Blockchain: Exploring the Potentials of Blockchain for Commons Governance." SSRN. 30 July 2018

RSA. "Bring-Your-Own-Identity Gains Steam in Information Security.” RSA. 13 April 2016, 
available at https://www.rsa.com/en-us/blog/2016-04/bring-your-own-identity-gainssteam-in-information-security [accessed 17 March 2019]

Sapien. Sapien: Decentralized Social News Platform (March 2018), available at https://www.sapien.network/static/pdf/SPNv1_3.pdf [accessed 17 March 2019]

Scott, Brett. "Visions of a Techno-Leviathan: The Politics of the Bitcoin Blockchain." Eth Zurich Center for Security Studies. 11 June 2014

Schmidt, Douglas. "Google Data Collection.” Digital Content Next. August 2018, available at https://digitalcontentnext.org/wp-content/uploads/2018/08/DCN-Google-DataCollection-Paper.pdf [accessed 17 March 2019]

Singh, Jagmeet. "Facebook Confirms Aadhaar Prompt for New Users, Says It Isn't Necessary." Gadgets 360. 17 December 2017, available at https:/gadgets.ndtv.com/socialnetworking/news/facebook-aadhaar-real-name-new-user-sign-up-onboarding-processtest-1792648 [accessed 17 March 2019]

State of the Dapps. What's a Dapp? (2019), available at https://www.stateofthedapps.com/whatsa-dapp.

Statista. "Number of social media users worldwide from 2010 to 2021 (in billions)." Statista. Available at https://www.statista.com/statistics/278414/number-of-worldwide-socialnetwork-users/ [accessed 17 March 2019]

Steemit. Steem: An incentivized, blockchain-based, public content platform (June 2018), available at https://steem.com/steem-whitepaper.pdf accessed 17 March 2019]

Swan, Melanie. Blockchain: Blueprint for a New Economy. Sebastopol: O’Reilly Media, 2015

Sumpter, David. Outnumbered: From Facebook and Google to Fake News and Filter-bubbles The Algorithms That Control Our Lives. Bloomsbury: Bloomsbury Publishing. 2018. Chapter 4

Tampuu, Piia \& Anu Masso. "'Welcome to the Virtual State': Estonian e-residency and the digitalised state as a commodity." European Journal of Cultural Studies 21 (5), 2018

Tapscott, Don \& Alex Tapscott. Blockchain Revolution: How the Technology Behind Bitcoin is Changing Money, Business, and the World. New York: Penguin, 2016

TechCrunch. "Decentralizing Everything with Ethereum's Vitalik Buterin | Disrupt SF 2017." Youtube video (18 September 2018), available at https://www.youtube.com/watch?v=WSN5BaCzsbo [accessed 17 March 2019]

The Better Identity Coalition. Better Identity in America: A Blueprint for Policymakers. July 2018, available at https://static1.squarespace.com/static/5a7b7a8490bade8a77c07789/t/5b4fe83b1ae6cfa99e 
58a05d/1531963453495/Better_Identity_Coalition+Blueprint+-+July+2018.pdf [accessed 17 March 2019]

Titcombe, James. "China's biggest social media company Tencent is now worth more than Facebook." The Telegraph. 21 November 2017, available at https://www.telegraph.co.uk/technology/2017/11/21/chinas-biggest-social-mediacompany-tencent-now-worth-facebook/ [accessed 17 March 2019]

Thatcher, Margaret. “A Life in Quotes.” The Guardian. 8 April 2013, available at https://www.theguardian.com/politics/2013/apr/08/margaret-thatcher-quotes [accessed 17 March 2019]

The European Parliament and the Council of the European Union. "On electronic identification and trust services for electronic transactions in the internal market and repealing Directive 1999/93/EC." Official Journal of the European Union 257, no. 73 (28 August 2014), available at https://eur-lex.europa.eu/legalcontent/EN/TXT/?uri=uriserv:OJ.L_.2014.257.01.0073.01.ENG [accessed 17 March 2019

The Sovrin Foundation. Sovrin ${ }^{\mathrm{TM}}$ : A Protocol and Token for Self-Sovereign Identity and Decentralized Trust." January 2018, available at https://sovrin.org/wpcontent/uploads/Sovrin-Protocol-and-Token-White-Paper.pdf [accessed 17 March 2019]

The Sovrin Foundation. The Inevitable Rise of Self-Sovereign Identity. 29 September 2016, available at https://www.evernym.com/wp-content/uploads/2017/07/The-Inevitable-Riseof-Self-Sovereign-Identity.pdf [accessed 17 March 2019]

The Sovrin Foundation. The Technical Foundations of Sovrin. Available at https://sovrin.org/wpcontent/uploads/2018/03/The-Technical-Foundations-of-Sovrin.pdf [accessed 17 March 2019]

Thomas, Elise. “Tagged, tracked and in danger: how the Rohingya got caught in the UN's risky biometric database." Wired. 12 March 2018, available at https:/www.wired.co.uk/article/united-nations-refugees-biometric-database-rohingyamyanmar-bangladesh [accessed 17 March 2019]

UN General Assembly. "Transforming Our World: the 2030 Agenda for Sustainable Development." 21 October 2015, A/RES/70/1, available at https://www.refworld.org/docid/57b6e3e44.html [accessed 17 March 2019]

UN General Assembly, Universal Declaration of Human Rights, 10 December 1948, 217 A (III), Article 6

Unite Ideas. Blockchain for Humanity Global Challenge. 2018, available at https://uniteideas.spigit.com/blockchain4humanity/Page/Home [accessed 17 March 2019] 
uPort. "First official registration of a Zug citizen on Ethereum." Medium. 15 November 2017, available at https://medium.com/uport/first-official-registration-of-a-zug-citizen-on-ethereum3554b5c2c238 [accessed 17 March 2019]

Veres One. The Veres One Project. 2018, available at https://veres.one/summary/ [accessed 17 March 2019]

Wang, Ge, Wei Zhang, and Runxi Zeng. "WeChat use intensity and social support: The moderating effect of motivators for WeChat use." Computers in Human Behaviour 91, 2019: $244-251$

Weber, Max. Weber's Rationalism and Modern Society, trans \& ed by Tony Waters and Dagmar Waters. London: Palgrave Books, 2015

Welsch, Chris. "Apple, Google, Microsoft, Facebook, Yahoo, and more deny providing direct access to PRISM surveillance program." The Verge. 6 June 2013, available at https://www.theverge.com/2013/6/6/4404112/nsa-prism-surveillance-apple-facebookgoogle-respond/in/4167369 [accessed 17 March 2019]

Wey, Alexandra. "Switzerland's first municipal blockchain vote hailed a success." Swiss Info. 2 July 2018, available at https://medium.com/uport/first-official-registration-of-a-zugcitizen-on-ethereum-3554b5c2c238 [accessed 17 March 2019]

William, Maxwell. "ERC-20 Tokens Explaine." CoinTelegraph. 12 May 2018, available at https://cointelegraph.com/explained/erc-20-tokens-explained [accessed 17 March 2019]

Windows Central. Microsoft and Mastercard Want to Give You a Universal Digital Identity: One Digital Identity to Rule Them All. 3 December 2018, available at https://www.windowscentral.com/microsoft-and-mastercard-want-give-you-universaldigital-identity

World Bank Development Report. “Enabling Digital Development: Digital Identity.” 2016, available at http://documents.worldbank.org/curated/en/896971468194972881/310436360_20160263 021000/additional/102725-PUB-Replacement-PUBLIC.pdf [accessed 17 March 2019]

World Bank Group. "Identification for Development." 2019, available at http://id4d.worldbank.org/about-us [accessed 19 March 2017]

World Bank Group and Center for Global Development. Principles on Identification: For $S$ Sustainable Development Toward the Digital Age. February 2017, available at http://documents.worldbank.org/curated/en/213581486378184357/pdf/112614REVISED-English-ID4D-IdentificationPrinciples-Folder-web-English-ID4DIdentificationPrinciples.pdf [accessed 17 March 2019]

World Bank. “Technology Landscape for Digital Identification.” 2018. Washington, DC: World 
Bank License: Creative Commons Attribution 3.0 IGO (CC BY 3.0 IGO), available at http://pubdocs.worldbank.org/en/199411519691370495/ID4DTechnologyLandscape.pdf [accessed 17 March 2019]

World Identity Network. "World Identity Network and United Nations team up to launch innovative blockchain pilot to help prevent child trafficking." PRNewswire. 10 November 2017, available at https:/www.prnewswire.com/news-releases/world-identity-networkand-united-nations-team-up-to-launch-innovative-blockchain-pilot-to-help-prevent-childtrafficking-300553921.html [accessed 17 March 2019] 